\title{
THE USE OF REPRESENTATIONS IN APPLIED HARMONIC ANALYSIS
}

\author{
FILIPPO DE MARI AND ERNESTO DE VITO
}

\section{TABle of Contents}

1. Representations of Lie groups. 1

1.1. Locally compact groups 2

1.2. Lie groups and Lie algebras 3

1.3. Representation theory 20

1.4. Square integrability 25

1.5. Unbounded operators 29

1.6. Stone's theorem and the differential of a representation. 31

2. The Heisenberg group and its representations 34

2.1. The group and its Lie algebra 34

2.2. The Schrödinger representation 38

2.3. Time-frequency analysis $\quad 41$

3. The metaplectc representation $\quad 45$

3.1. More on the symplectic group $\quad 45$

3.2. Construction of the metaplectic representation 47

3.3. Restriction to triangular subgroups 50

$\begin{array}{ll}\text { Bibliografy } & 59\end{array}$

\section{Representations of Lie GROUPS.}

Representation theory of groups is a vast subject. Many of the aspects of this theory that are of interest in Harmonic Analysis, and, in particular, of that body of ideas and techniques that might be collectively referred to as Applied Harmonic Analysis, can be studied within the class of locally compact and second countable topological groups, a family whose nickname in the group theory jargon is "lcsc". Most interesting examples in which we are interested, however, belong to the smaller and nicer class of Lie groups, which feature an additional geometric-analytic nature that allows, for instance, to speak about dimension or to perform handy calculations such as taking derivatives or solving differential equations. As it is often the case, there is a tradeoff between the beautiful generality and formal simplicity of lcsc groups, for which a limited number of techniques is available, and the class of Lie groups, which is harder to define, but enjoys many more desirable properties. At the level of practical 
examples, the theoretical obstacles fade out almost completely because one is in the end dealing with matrix groups whose description in coordinates is often quite natural, and the computations that are at times hard to formalize in the general setup are simple extentions of those that everyone is familiar with in $\mathbb{R}^{d}$. What is gained is a general conceptual landscape that provides insight and makes full use of the simmetries that are involved in the problems at hand. Finally, we believe that research in this area requires a wide box of mathematical tools, including those that are pertinent to Lie groups, because of their effectiveness, flexibility and depth.

For these reasons we shall work mostly within this family, even though we by no means use the full force of the representation theory of Lie groups. On the reader's side, we take for granted some working knowledge of topology, calculus, linear algebra and elementary differential geometry. From the latter, we essentially need the notion of smooth manifold, the basic constructs of tangent vectors and vector fields and the use of tangent mappings, or differentials, that we actually very quickly review. Some of the results concerning Lie groups that are summarized in Section 1.2 below are used in the sections that follow, others are presented in order to achieve a better understanding of the main ideas.

1.1. Locally compact groups. We start with some fundamental definitions and results. For a detailed account on these matters the reader may consult [14].

Definition 1.1. A topological group is a group $G$ endowed with a topology relative to which the group operations

$$
(g, h) \mapsto g h, \quad g \mapsto g^{-1}
$$

are continuous as maps $G \times G \rightarrow G$ and $G \rightarrow G$, respectively. $G$ is locally compact if every point has a compact neighborhood. We shall also assume our groups to be Hausdorff.

Definition 1.2. A Borel measure $\mu$ defined on the $\sigma$-algebra generated by the open sets of the topological space $X$ is called a Radon measure if:

(i) it is finite on compact sets;

(ii) it is outer regular on the Borel sets: for every Borel set E

$$
\mu(E)=\inf \{\mu(U): U \supset E, U \text { open }\}
$$

(iii) it is inner regular on the open sets: for every open set $U$

$$
\mu(U)=\sup \{\mu(K): K \subset E, K \text { compact }\} .
$$

Definition 1.3. A left Haar measure on the topological group $G$ is a non zero Radon measure $\mu$ such that $\mu(x E)=\mu(E)$ for every Borel set $E \subset G$ and every $x \in G$. Similarly for right Haar measures.

Of course, the prototype of Haar measure is the Lebesgue measure on the additive group $\mathbb{R}^{d}$, which is invariant under left (and right) translations.

Theorem 1.4. Every locally compact group $G$ has a left Haar measure $\lambda$, which is essentially unique in the sense that if $\mu$ is any other left Haar measure, then there exists a positive constant $C$ such that $\mu=c \lambda$. 
If we fix a left Haar measure $\lambda$ on $G$, then for any $x \in G$ the measure $\lambda_{x}$ defined by

$$
\lambda_{x}(E)=\lambda(E x)
$$

is again a left Haar measure. Therefore there must exist a positive real number, denoted $\Delta(x)$ such that

$$
\lambda_{x}=\Delta(x) \lambda .
$$

The function $\Delta: G \rightarrow \mathbb{R}_{+}$is called the modular function.

Proposition 1.5. Let $G$ be a locally compact group. The modular function $\Delta: G \rightarrow \mathbb{R}_{+}$ is a continuous homomorphism into the multiplicative group $\mathbb{R}_{+}$. Furthermore, for every $f \in L^{1}(G)$ we have

$$
\int_{G} f(x y) d x=\Delta(y)^{-1} \int_{G} f(x) d x .
$$

In the Section 1.2.8 below we give some examples in the context of Lie groups. A group for which for which the modular function is identically equal to one, is called unimodular. Large classes of groups are unimodular, such as the Abelian, compact, nilpotent, semisimple and reductive groups. Nevetherless, in Applied Harmonic Analysis non-unimodular groups play a prominent role, such as the affine group " $a x+b$ " that we define in the next section.

1.2. Lie groups and Lie algebras. We recall, without proofs, some basic facts about Lie groups and Lie algebras. For a concise and effective exposition, see [23]. Classical references with a wider scope are [17] and [22]. We shall often use the word "smooth" in place of " $C$ " "

Definition 1.6. A Lie group $G$ is a smooth manifold endowed with a group structure such that the group operations $(g, h) \mapsto g h$ and $g \mapsto g^{-1}$ are smooth.

Example 1.7. Clearly, $\mathbb{R}^{d}$ is an additive, Abelian Lie group. Similarly $\mathbb{C}^{d}$, identified with $\mathbb{R}^{2 d}$ as manifolds. Any real or complex vector space can be given the structure of Lie group simply choosing a basis and then identifying with $\mathbb{R}^{d}$.

Example 1.8. The sphere $S^{1}=\left\{e^{i \theta}: \theta \in[0,2 \pi)\right\}$ is an Abelian compact Lie group.

Example 1.9. The multiplicative group $G L(d, \mathbb{R})$ of invertible matrices is a Lie group. Indeed, it is an open submanifold of $\mathbb{R}^{d^{2}}$ with the global coordinates $x_{i j}$ that assign to a matrix its $i j$-th entry. If $y, z \in G L(d, \mathbb{R})$, then $x_{i j}(y z)$ and $x_{i j}\left(y^{-1}\right)$ are rational functions of $\left\{x_{i j}(y), x_{i j}(z)\right\}$ and of $\left\{x_{i j}(y)\right\}$, respectively, with non vanishing denominator. Hence they are smooth functions.

Great attention deserve the closed subgroups of $G L(d, \mathbb{R})$. They are automatically Lie groups, and in fact enjoy additional nice features from the topological point of view. This very important result is due to Cartan and is recalled below in Theorem 1.26.

A remarkable closed subgroup of $G L(d, \mathbb{R})$ is the symplectic group $S p(d, \mathbb{R})$, that plays an important role in this article. It is defined by

$$
S p(d, \mathbb{R})=\left\{g \in G L(d, \mathbb{R}):{ }^{t} g J g=J\right\}
$$


where ${ }^{t} g$ is the transpose of $g$ and where $J$ is the canonical skew-symmetric matrix

$$
J=\left[\begin{array}{cc}
0 & I_{d} \\
-I_{d} & 0
\end{array}\right],
$$

that defines the standard symplectic form (see Section 3.1 for more details). Notice that for $d=1$ we have $S p(1, \mathbb{R})=S L(2, \mathbb{R})$, the latter being the group of $2 \times 2$ real matrices with deteminant equal to one.

Example 1.10. The affine group " $a x+b$ ". There are several possible versions of this group. Let $G=\mathbb{R}_{+} \times \mathbb{R}$ as a manifold. One can visualize it as the right half plane. The multiplication is obtained by thinking of the pair $(a, b)$, with $a>0$ and $b \in \mathbb{R}$, as identifying the affine transformation of the real line given by $x \mapsto a x+b$, whence the name. The composition of maps

$$
x \mapsto a x+b \mapsto a^{\prime}(a x+b)+b^{\prime}=\left[a^{\prime} a\right] x+\left[a^{\prime} b+b\right]
$$

yields the product rule

$$
\left(a^{\prime}, b^{\prime}\right)(a, b)=\left(a^{\prime} a, a^{\prime} b+b^{\prime}\right) .
$$

Evidently, both functions $a^{\prime} a$ and $a^{\prime} b+b$ are smooth in the global coordinates on $G$, which is then a Lie group. Evidently, $G$ is connected. When speaking of the " $a x+b$ " group we refer to this group.

A non-connected version arises by taking $a \in \mathbb{R}^{*}=\mathbb{R} \backslash\{0\}$ instead of $a>0$. We shall refer to this as the full affine group. Yet another slightly different construction comes from thinking of the pair $(a, b)$ as identifying the affine transformation $x \mapsto a(x+b)$. This point of view yields both a connected and a non-connected Lie group.

Definition 1.11. A Lie algebra $\mathfrak{g}$ over $\mathbb{R}$ is a real vector space endowed with a bilinear operation $[\cdot, \cdot]: \mathfrak{g} \times \mathfrak{g} \rightarrow \mathfrak{g}$, called bracket, such that

i) $[X, Y]=-[Y, X]$ for every $X, Y \in \mathfrak{g}$,

ii) $[X,[Y, Z]]=[[X, Y], Z]+[Y,[X, Z]]$ for every $X, Y, Z \in \mathfrak{g}$.

Item ii), oterwise known as the Jacobi identity, should be thought of as an analogous version of the derivative of the product. Indeed, if for any $X \in \mathfrak{g}$ we put

$$
\operatorname{ad} X: \mathfrak{g} \rightarrow \mathfrak{g}, \quad \operatorname{ad} X(Y)=[X, Y]
$$

the Jacobi identity may be formulated:

$$
\operatorname{ad} X([Y, Z])=[\operatorname{ad} X(Y), Z]+[Y, \operatorname{ad} X(Z)],
$$

which reminds the derivative of the product, with the bracket as product. This seemingly awkward notation comes from the fact that the map $X \mapsto$ ad $X$ defines the so-called adjoint representation (see Subsection 1.2.6 below for details).

Example 1.12. If $V$ is a vector space, the set $\operatorname{End}(V)$ of all linear maps of $V$ into itself is a Lie algebra under the commutator $[\phi, \psi]=\phi \psi-\psi \phi$ as bracket. With this structure understood, it is denoted by $\mathfrak{g l}(V)$. 
It should be clear what is meant by Lie subalgebra of a Lie algebra $\mathfrak{g}$ : it is a vector subspace $\mathfrak{h}$ which is closed under bracket, that is, such that if $A, B \in \mathfrak{h}$ then $[A, B] \in \mathfrak{h}$. A stronger notion is that of $i d e a l$ : it is a Lie subalgebra $\mathfrak{h}$ of $\mathfrak{g}$ with the stronger property that $[\mathfrak{h}, \mathfrak{g}] \subset \mathfrak{h}$, which means that for every $A \in \mathfrak{h}$ and every $B \in \mathfrak{g}$ we have $[A, B] \in \mathfrak{h}$.

1.2.1. Tangent vectors and vector fields. Tangent vectors can be defined in several equivalent ways. A natural way to think of a tangent vector at the point $p$ of the manifold $M$ is to introduce an equivalence relation among all the smooth curves $c$ defined in some open interval containing $0 \in \mathbb{R}$ with values in $M$ such that $c(0)=p$. We establish that $c_{1} \sim c_{2}$ if for every smooth function $f: \mathcal{U}_{p} \rightarrow \mathbb{R}$ it holds

$$
\left.\frac{d}{d t}\right|_{t=0} f\left(c_{1}(t)\right)=\left.\frac{d}{d t}\right|_{t=0} f\left(c_{2}(t)\right)
$$

where $\mathcal{U}_{p}$ is an open neighborhood of $p$ in $M$. The equivalence class $\langle c\rangle_{p}$ of any of these curves is then a tangent vector to $M$ at $p$. This line of thoughts will be adopted in Section 2 when computing the generators of the Lie algebra of the Heisenberg group. The set of all tangent vectors at $p$ is a vector space, the tangent space of $M$ at $p$, denoted $T_{p}(M)$. Once a coordinate patch $\left(\mathcal{U}_{p}, x^{1}, \ldots, x^{d}\right)$ around $p \in M$ has been fixed, the equivalence class of the special curve $c_{i}$ defined by $t \mapsto\left(p_{1}, \ldots, p_{i}+t, \ldots, p_{d}\right)$ is identified with the tangent vector denoted by

$$
\left.\frac{\partial}{\partial x^{i}}\right|_{p}
$$

because it operates on any function $f$ defined and smooth on $\mathcal{U}_{p}$ by

$$
\left.\frac{\partial}{\partial x^{i}}\right|_{p} f=\left.\frac{d}{d t}\right|_{t=0} f\left(c_{i}(t)\right)
$$

the common value attained along all curves equivalent to $c_{i}$. These particular tangent vectors give rise to a basis of the vector space $T_{p}(M)$. A sensible expression for a tangent vector at $p$ is therefore

$$
X_{p}=\left.\sum_{i=1}^{d} a_{i} \frac{\partial}{\partial x^{i}}\right|_{p} \in T_{p}(M),
$$

with $a_{1}, \ldots, a_{d} \in \mathbb{R}$. As implicitly said in the previous paragraph, a tangent vector acts on a function $\psi$ defined locally around $p$ as a first order differential operator and produces a real number, the effect of the derivative $X_{p} \psi$ defined in (1.4). Using equivalence classes of curves, this real number is

$$
\langle c\rangle_{p} f=\left.\frac{d}{d t}\right|_{t=0} f(c(t)) .
$$

A vector field $X$ on the manifold $M$ is a smooth map that assigns to each point $p \in M$ a tangent vector at that point, that is, an element $X_{p} \in T_{p}(M)$. The simplest example of a locally defined vector field is

$$
\frac{\partial}{\partial x^{i}},\left.\quad p \mapsto \frac{\partial}{\partial x^{i}}\right|_{p} .
$$


Vector fields act on functions, in the sense that if $X$ is a vector field and $\psi$ is a function, then $X \psi$ is the function that at the point $p$ takes the value

$$
X \psi(p)=X_{p}(\psi) .
$$

The smoothness of $X$ is, by definition, the requirement that $X \psi$ is smooth whenever $\psi$ is such. Let $\mathfrak{X}(M)$ denote the set of all smooth vector fields on $M$. They can be interpreted as the derivations of $C^{\infty}(M)$, the algebra of smooth functions. This means that any $X \in \mathfrak{X}(M)$ acts on smooth functions linearly (that is, $X(\alpha \varphi+\beta g)=$ $\alpha X(\varphi)+\beta X(\psi)$ for any choice of scalars $\alpha$ and $\beta$ ), producing new smooth functions, and that the derivative rule

$$
X(\varphi \psi)=X(\varphi) \psi+\varphi X(\psi)
$$

holds. As a consequence, in any local coordinate system $\left(\mathcal{U}, x^{1}, \ldots, x^{d}\right)$ a vector field $X \in \mathfrak{X}(M)$ can be expressed as

$$
X=\sum_{i=1}^{d} f_{i} \frac{\partial}{\partial x^{i}}
$$

where $f_{1}, \ldots, f_{d} \in C^{\infty}(M)$. The action of $X$ on a function $\psi \in C^{\infty}(M)$ is then the function $X \psi$ whose value at $p \in \mathcal{U} \subset M$ is

$$
X \psi(p)=\sum_{i=1}^{d} f_{i}(p) \frac{\partial \psi}{\partial x^{i}}(p)
$$

Thus $X$ is smooth if and only if the functions $f_{i}$ are smooth.

1.2.2. Lie algebras of vector fields. The set $\mathfrak{X}(M)$ enjoys a structural algebraic property, it is a $C^{\infty}(M)$-module. This means that the vector fields form an Abelian group under the natural (pointwise) sum, and they can be multiplied (pointwise) by smooth functions, respecting the rules that modules require, namely

$$
f(X+Y)=f X+f Y, \quad(f+g) X=f X+g X, \quad f(g X)=(f g) X, \quad 1 X=X,
$$

where $X, Y \in \mathfrak{X}(M), f, g \in C^{\infty}(M)$ and 1 is the function equal to one on $M$. More remarkably, $\mathfrak{X}(M)$ is a (typically infinite dimensional) Lie algebra under the commutator. This is a consequence of the fact that

$$
[X, Y]:=X \circ Y-Y \circ X
$$

is in fact a first order differential operator because the second order terms vanish due to the equality of mixed partials. Therefore $[X, Y] \in \mathfrak{X}(M)$ and the Jacobi identity is readily established, together with bilinearity and skew-symmetry. As we shall see below, the main feature of Lie groups is that, thanks to the presence of (left) translations, the Lie algebra $\mathfrak{X}(G)$ always admits a very natural finite dimensional Lie subalgebra.

Any smooth map $F: M \rightarrow N$ between smooth manifolds gives rise to the tangent map $F_{*}$ of the corresponding tangent bundles. Thus, for any $p \in M$ the tangent map at $p$, also known as the differential of $F$ at $p$, is the linear map $F_{* p}: T_{p}(M) \rightarrow T_{F(p)}(N)$ carrrying the tangent vector $X_{p} \in T_{p}(M)$ to the tangent vector $F_{* p} X_{p} \in T_{F(p)}(N)$ whose action (as a derivation) on a function $\psi$ defined in a neighborhood of $F(p)$ is

$$
F_{* p} X_{p}(\psi)=X_{p}(\psi \circ F) .
$$


When $M$ and $N$ are open subsets of $\mathbb{R}^{d}$ and $\mathbb{R}^{n}$, respectively, the differential is expressed in the canonical bases by the $n \times d$ Jacobian matrix.

Let now $G$ be a Lie group and denote by $l_{g}: G \rightarrow G$ the left translation by $g \in G$, that is $l_{g}(h)=g h$. A vector field $X \in \mathfrak{X}(G)$ is called left invariant on $G$ if for every $g, h \in G$ it satisfies

$$
\left(l_{g}\right)_{* h} X_{h}=X_{g h}
$$

The set of all left invariant vector fields on $G$ will be denoted $\mathcal{L}(G)$.

Proposition 1.13. Let $G$ be a Lie group and denote by $\mathcal{L}(G)$ the set of left invariant vector fields on $G$. Then:

i) $\mathcal{L}(G)$ is an $\mathbb{R}$-vector space and the linear map $\alpha: \mathcal{L}(G) \rightarrow T_{e}(G)$ defined by $\alpha(X)=X_{e}$ is a vector space isomorphism between $\mathcal{L}(G)$ and the tangent space to $G$ at the identity $e \in G$. Consequently, $\operatorname{dim} \mathcal{L}(G)=\operatorname{dim} T_{e}(G)=\operatorname{dim} G$.

ii) The commutator $[X, Y]=X \circ Y-Y \circ X$ of two left invariant vector fields is again a left invariant vector field. With this bracket, $\mathcal{L}(G)$ becomes a Lie algebra, which will be called the Lie algebra of $G$.

We shall now discuss in some detail an example that plays a crucial role in what follows and illustrates the concepts that we have just introduced. Perhaps the most important Lie group in which we are interested is $G L(d, \mathbb{R})$, the invertible $d \times d$ matrices. Indeed, as we shall see, most of the groups at which we look in this article arise as closed subgroups of $G L(d, \mathbb{R})$. Therefore, $G L(d, \mathbb{R})$ serves as a large ambient group in which the action takes place. We shall now see that its Lie algebra is naturally identified with the Lie algebra of all $d \times d$ matrices. The identification that we present provides the most basic insight when dealing with matrix Lie groups.

Let us denote by $\mathfrak{g l}(d, \mathbb{R})$ the Lie algebra whose underlying vector space is the set $M_{d}(\mathbb{R})$ of square $d \times d$ matrices with real entries and whose bracket is the commutator $[A, B]=A B-B A$. The reader may check that this is indeed a bracket, in the sense that it defines a bona fide Lie algebra structure on $M_{d}(\mathbb{R})$. If we use global coordinates on $G L(d, \mathbb{R})$ as an open subset of $\mathbb{R}^{d^{2}}$ (se Example 3 above), then a tangent vector to $G L(d, \mathbb{R})$ at $g \in G L(d, \mathbb{R})$ may be written as

$$
\xi=\left.\sum_{i j} a_{i j} \frac{\partial}{\partial x_{i j}}\right|_{g} \in T_{g}(G L(d, \mathbb{R}))
$$

for some $d^{2}$ real numbers $a_{i j}$. If $h \in G L(d, \mathbb{R})$, then the image of $\xi$ under the differential $\left(l_{h}\right)_{* g}$ is the tangent vector

$$
\left(l_{h}\right)_{* g} \xi=\left.\sum_{i j} b_{i j} \frac{\partial}{\partial x_{i j}}\right|_{h g} \in T_{h g}(G L(d, \mathbb{R})),
$$

where again $b_{i j}$ are $d^{2}$ suitable real numbers . In order to compute the explicit value of these coordinates as functions of $a_{i j}$ and of $h$, we use the fundamental identification of a tangent vectors as first order differential operators discussed above and evaluate 
the vector field $\left(l_{h}\right)_{* g} \xi$ on the the coordinate function $x_{i j}$. Explicitely, we get

$$
\begin{aligned}
b_{i j} & =\left(\left(l_{h}\right)_{* g} \xi\right)\left(x_{i j}\right) \\
& =\xi\left(x_{i j} \circ l_{h}\right) \\
& =\xi\left(\sum_{k} h_{i k} x_{k j}\right) \\
& =\left.\sum_{p q} a_{p q} \frac{\partial}{\partial x_{p q}}\right|_{g}\left(\sum_{k} h_{i k} x_{k j}\right) \\
& =\sum_{p q} a_{p j} h_{i p} \\
& =(h A)_{i j},
\end{aligned}
$$

where $A=\left(a_{i j}\right)$ is the $d \times d$ real matrix associated to the components $a_{i j}$. Hence

$$
\left(l_{h}\right)_{* g} \xi=\left.\sum_{i j}(h A)_{i j} \frac{\partial}{\partial x_{i j}}\right|_{h g} .
$$

This formula suggests an expression for the left invariant vector fields on $G L(d, \mathbb{R})$. We allude to the following: a left invariant vector field on $G L(d, \mathbb{R})$ is completely determined by its value at the identity, whose coordinates are encoded by a $d \times d$ matrix, say $A$. We denote by $X^{A}$ such vector field. As the next proposition shows, the correpondence $A \leftrightarrow X^{A}$ is an instance of the isomorphism described in item (i) of Proposition 1.13. The isomorphism is very nice and operative, in the sense that the bracket of the vector fields $X^{A}$ and $X^{B}$ is the vector field $X^{A B-B A}$, so that we can completely identify the left invariant vector fields on $G L(d, \mathbb{R})$ with the elements of Lie algebra $\mathfrak{g l}(d, \mathbb{R})$ and compute directly with matrices instead of going through complicated expressions that involve partial derivates.

Proposition 1.14. Given any matrix $A \in M_{n}(\mathbb{R})$, the vector field $X^{A}$ on $G L(d, \mathbb{R})$ whose value at $g \in G L(d, \mathbb{R})$ is

$$
X_{g}^{A}=\left.\sum_{i j}(g A)_{i j} \frac{\partial}{\partial x_{i j}}\right|_{g}
$$

is a left invariant vector field on $G L(d, \mathbb{R})$. Further, the map $A \mapsto X^{A}$ is a Lie algebra isomorphism between $\mathfrak{g l}(d, \mathbb{R})$ and the Lie algebra of $G L(d, \mathbb{R})$.

Proof. The fact that $X^{A}$ is a left invariant vector field follows at once from (1.5). Take now $A, B \in \mathfrak{g l}(d, \mathbb{R})$. We now find the component of $\left[X^{A}, X^{B}\right]_{g}$ by computing 
$\left(X^{A} \circ X^{B}-X^{B} \circ X^{A}\right)_{g}\left(x_{i j}\right)$. Since

$$
\begin{aligned}
\left(X^{A} \circ X^{B}\right)_{g}\left(x_{i j}\right) & =\left.\sum_{p q}(g A)_{p q} \frac{\partial}{\partial x_{p q}}\right|_{g}\left(\left.\sum_{m n}(g B)_{m n} \frac{\partial}{\partial x_{m n}}\right|_{g}\left(x_{i j}\right)\right) \\
& =\left.\sum_{p q}(g A)_{p q} \frac{\partial}{\partial x_{p q}}\right|_{g}(g B)_{i j} \\
& =\left.\sum_{p q}(g A)_{p q} \frac{\partial}{\partial x_{p q}}\right|_{g}\left(\sum_{k} g_{i k} B_{k j}\right) \\
& =\sum_{p q}(g A)_{p q}\left(\sum_{k} \delta_{i p} \delta_{q k} B_{k j}\right) \\
& =\sum_{q}(g A)_{i q} B_{q j} \\
& =(g A B)_{i j},
\end{aligned}
$$

we get

$$
\left(X^{A} \circ X^{B}-X^{B} \circ X^{A}\right)_{g}\left(x_{i j}\right)=(g(A B-B A))_{i j}
$$

and cosequently

$$
\left[X^{A}, X^{B}\right]=X^{[A, B]},
$$

which is precisely what we wanted to show.

1.2.3. Homomorphisms. Take two Lie groups, $G$ ed $H$. A map $\varphi: G \rightarrow H$ is a Lie group homomorphism if it is a group homomorphism (hence if $\varphi(x y)=\varphi(x) \varphi(y)$ for every $x, y \in G$ and if $\varphi(e)=e$, the identities of $G$ and $H$, respectively) and also a smooth map of manifolds. We say that $\varphi$ is a Lie group isomorphism if it is a diffeomorphism, that is, a smooth bijection with smooth inverse.

Example 1.15. A good example of homomorphism is the map $\varphi: U(2) \rightarrow S p(2, \mathbb{R})$ defined by

$$
\varphi(X+i Y)=\left[\begin{array}{cc}
X & Y \\
-Y & X
\end{array}\right]
$$

Here $U(2)$ stands for the $2 \times 2$ complex unitary matrices, those for which ${ }^{t} \bar{g} g=I$. Now, if $g=X+i Y$ with $X, Y \in M_{2}(\mathbb{R})$, then ${ }^{t} \bar{g} g=I$ is equivalent to ${ }^{t} X X+{ }^{t} Y Y=I$ and ${ }^{t} X Y$ symmetric. But then $\varphi(X+i Y)$ satisfies

$$
\begin{aligned}
{ }^{t} \varphi(X+i Y) J \varphi(X+i Y) & =\left[\begin{array}{cc}
{ }^{t} X & -{ }^{t} Y \\
{ }^{t} Y & { }^{t} X
\end{array}\right]\left[\begin{array}{cc} 
& I \\
-I &
\end{array}\right]\left[\begin{array}{cc}
X & Y \\
-Y & X
\end{array}\right] \\
& =\left[\begin{array}{ll}
-{ }^{t} X Y+{ }^{t} Y X & { }^{t} X X+{ }^{t} Y Y \\
-{ }^{t} Y Y-{ }^{t} X X & { }^{t} Y X-{ }^{t} X Y
\end{array}\right]=J
\end{aligned}
$$

and is therefore symplectic. This proves that $\varphi$ takes values in $S p(2, \mathbb{R})$. It is easy to see that $\varphi(g h)=\varphi(g) \varphi(h)$ whereas $\varphi(I)=I$ is obvious. As for smoothness, this is a somewhat tricky issue that needs not concern us now. Finally, $U(2)$ has dimension 4, while $S p(2, \mathbb{R})$ has dimension 10 , so $\varphi$ cannot possibly be an isomorphism. 
An isomorphism of $G$ onto itself is called an automorphism. A natural class of automorphisms are the so-called inner automorphisms, namely those given by inner conjugation. If $g \in G$, the inner conugation defined by $g$ is the map

$$
i_{g}: G \rightarrow G, \quad i_{g}(h)=g h g^{-1} .
$$

In general, a Lie group posesses automorphisms that are not inner. A good example is given by the automorphisms of the Heisenberg group, that are listed below in Theorem 2.8 .

Observe that the set of linear automorphisms of a finite dimensional $\mathbb{R}$-vector space $V$ (hence a Lie group) has itself a natural structure of Lie group, because if a basis is selected, then the group of all linear invertible maps may be identified with the Lie group of invertible matrices. This group is denoted by $\operatorname{Aut}(V)$. As we shall see in Section 1.3, a homomorphism $\pi: G \rightarrow \operatorname{Aut}(V)$ is what is called a finite dimensional representation of $G$.

If $\mathfrak{g}$ and $\mathfrak{h}$ are both real Lie algebras, a linear map $\psi: \mathfrak{g} \rightarrow \mathfrak{h}$ for which $\psi([X, Y])=$ $[\psi(X), \psi(Y)]$ is a Lie algebra homomorphism. Further, if $\psi$ is a linear isomorphism, then it is called a Lie algebra isomorphism. If $\mathfrak{h}=\mathfrak{g l}(W)$ is the Lie algebra of all endomorphisms of a vector space $W$, a Lie algebra homomorphism $\psi: \mathfrak{g} \rightarrow \mathfrak{h}$ is called a representation of $\mathfrak{g}$ on $W$. This is the case of the homomorphism $X \mapsto \operatorname{ad} X$, which defines the adjoint representation of $\mathfrak{g}$, a representation of $\mathfrak{g}$ on itself.

Let $\varphi: G \rightarrow H$ be a Lie group homomorphism. Its differential evaluated at the identity $\varphi_{* e}: T_{e}(G) \rightarrow T_{e}(H)$ is a linear map. By the natural identifications $T_{e}(G) \simeq$ $\mathcal{L}(G)$ and $T_{e}(H) \simeq \mathcal{L}(H), \varphi_{* e}$ induces a linear map $\mathcal{L}(G) \rightarrow \mathcal{L}(H)$ denoted $d \varphi$. More precisely, if $X \in \mathcal{L}(G)$, then $d \varphi(X)$ is the unique left invariant vector field on $G$ such that

$$
(d \varphi(X))_{e}=\varphi_{* e} X_{e}
$$

The following result clarifies matters.

Proposition 1.16. Let $\varphi: G \rightarrow H$ be a Lie group homomorphism. Then $\varphi_{* g} X_{g}=$ $(d \varphi(X))_{\varphi(X)}$ for every $X \in \mathcal{L}(G)$ and $d \varphi$ is a Lie algebra homomorphism.

Take again a Lie group homomorphism $i: G \rightarrow H$ and assume that $i$ is injective and that also its differential is injective at every point (such a map is called an injective immersion). In this case the pair $(i, H)$ is called a Lie subgroup of $G$. It should be clear that whenever a Lie subgroup is given, then, upon taking the differential $d i$ of the corresponding immersion, one gets an immersion of Lie algebras. In other words, to any Lie subgroup there corresponds a Lie subalgebra. The question concerning a possible reverse correspondence is addressed by the following fundamental result.

Theorem 1.17. Let $G$ be a Lie group with Lie algebra $\mathfrak{g}$ and take a Lie subalgebra $\mathfrak{h}$ of $\mathfrak{g}$. Then there exists a connected Lie subgroup $(i, H)$ of $G$, unique up to isomorphisms, such that $\operatorname{di}(\mathcal{L}(H))=\mathfrak{h}$. Therefore there is a bijective correspondence between the connected Lie subgroup of a Lie group and the subalgebras of its Lie algebra. Under this bijection, normal subgroups correspond to ideals. 
The theory of covering groups is also very important, and relevant in the present context, but we content ourselves with the observation that every connected Lie group has a simply connected covering that admits the structure of Lie group and for which the covering homomorphism is a Lie group homomorphism. Furthermore, a Lie group homomorphism $\varphi: G \rightarrow H$ is a covering map if and only if $d \varphi$ is a Lie algebra isomorphism. The following theorem is of central importance in the theory of Lie groups, it is the monodromy principle for Lie groups, namely the possibility of lifting homomorphisms from the Lie algebra to the Lie group.

Theorem 1.18. Let $G_{1}$ and $G_{2}$ be two Lie groups with Lie algebras $\mathfrak{g}_{1}$ and $\mathfrak{g}_{2}$, respectively, and let $\lambda: \mathfrak{g}_{1} \rightarrow \mathfrak{g}_{2}$ be a Lie algebra homomorphism. Then there cannot be more than one Lie group homomorphism $\varphi: G_{1} \rightarrow G_{2}$ such that $d \varphi=\lambda$. If $G_{1}$ is simply connected, then such a $\varphi$ exists.

1.2.4. Exponential mapping. We now review in some detail the definition of the fiundamental map linking the Lie group with its Lie algebra, namely the exponential mapping $\exp : \mathfrak{g} \rightarrow G$. Let $\mathbb{R}$ be the additive Lie group of real numbers. Its Lie algebra is onedimensional and is generated by the vector field $\frac{d}{d t}$. Take now a Lie group $G$ with Lie algebra $\mathfrak{g}$, and fix $X \in \mathfrak{g}$. The map

$$
\tau \frac{d}{d t} \mapsto \tau X, \quad \tau \in \mathbb{R}
$$

is a Lie algebra homomorphism from $\mathbb{R}$ into $\mathfrak{g}$. Since $\mathbb{R}$ is simply connected, there exists a unique homomorphism $\xi_{X}: \mathbb{R} \rightarrow G$ such that:

$$
\left\{\begin{array}{l}
\left.\left(\xi_{X}\right)_{* \tau} \frac{d}{d t}\right|_{t=\tau}=X_{\xi_{X}(\tau)} \\
\left.\left(\xi_{X}\right)_{* 0} \frac{d}{d t}\right|_{t=0}=X_{e}
\end{array}\right.
$$

Conversely, if $\eta: \mathbb{R} \rightarrow G$ is a Lie group homomorphism, then $X=d \eta\left(\frac{d}{d t}\right)$ satisfies $\eta=\xi_{X}$. Hence, the correspondence $X \mapsto \xi_{X}$ establishes a bijection between $\mathfrak{g}$ and the set of homomorphisms from $\mathbb{R}$ into $G$ with the property that $d \xi_{X}\left(\frac{d}{d t}\right)=X$ for every $X \in \mathfrak{g}$.

Fix now $\tau \in \mathbb{R}$ and $X \in \mathfrak{g}$. Then, if $m_{\tau}$ denotes the multiplication by $\tau$ in $\mathbb{R}$, the map $\eta(t)=\xi_{X}(\tau t)=\xi_{X} \circ m_{\tau}(t)$ is again a homomorphism from $\mathbb{R}$ into $G$ and since

$$
\left.\eta_{* 0} \frac{d}{d t}\right|_{t=0}=\left.\left(\xi_{X}\right)_{* 0} \tau \frac{d}{d t}\right|_{t=0}=\tau X_{e}
$$

it follows that $\eta=\xi_{\tau X}$, that is

$$
\xi_{\tau X}(t)=\xi_{X}(\tau t), \quad t, \tau \in \mathbb{R}, X \in \mathfrak{g} .
$$

We define

$$
\exp X=\xi_{X}(1), \quad X \in \mathfrak{g} .
$$

The map exp $: \mathfrak{g} \rightarrow G$ is called the exponential mapping. From (1.8) it follows that

$$
\begin{aligned}
& \xi_{X}(t)=\exp (t X), \quad t \in \mathbb{R}, X \in \mathfrak{g} \\
& \exp 0=e .
\end{aligned}
$$


It is easy to check that if $X \in \mathfrak{g}$ e $x \in G$ are fixed, the map $t \mapsto x \exp (t X)$ defines the integral curve relative to $X$ passing through $x$, namely the smooth curve whose differential carries the tangent vector in $\mathbb{R}$ to the value of the vector field at the image point. Hence, for every $C^{\infty}$-function $f$ in a neighborhood of $x$ we have

$$
X_{x}(f)=\left.\frac{d}{d t}\right|_{t=0} f(x \exp t X) .
$$

An immediate consequence of the fact that $\xi_{X}$ is a homomorphism are the formulae

$$
\begin{aligned}
\exp (t+s) X & =\exp t X \exp s X \\
\exp (-t X) & =(\exp t X)^{-1} .
\end{aligned}
$$

The following formulae require some harder work:

$$
\begin{aligned}
& \exp t X \exp t Y=\exp \left\{t(X+Y)+\frac{1}{2} t^{2}[X, Y]+O\left(t^{3}\right)\right\} \\
& \exp (-t X) \exp (-t Y) \exp t X \exp t Y=\exp \left\{t^{2}[X, Y]+O\left(t^{3}\right)\right\} \\
& \exp t X \exp t Y \exp (-t X)=\exp \left\{t Y+t^{2}[X, Y]+O\left(t^{3}\right)\right\} .
\end{aligned}
$$

Formula (1.13) is the well-known Baker-Campbell-Hausdorff formula. The exponential map is in general neither injective nor surjective, but it is locally very nice:

Proposition 1.19. The exponential map is $C^{\infty}$ and its differential at zero is the identity map of $\mathfrak{g}$. Consequently, exp establishes a diffeomorphism of a neighborhood of $0 \in \mathfrak{g}$ onto a neighborhood of $e \in G$.

One of the most fundamental properties of the exponential mapping is that it always intertwines the homomorphisms of Lie groups with the corresponding homomorphisms of the Lie algebras:

Theorem 1.20. Let $\varphi: G \rightarrow H$ be a Lie group homomorphism with differential $d \varphi: \mathfrak{g} \rightarrow \mathfrak{h}$. Then, for every $X \in \mathfrak{g}$

$$
\varphi(\exp X)=\exp (d \varphi X) .
$$

By means of the previous result it is easy to show the next one, which is of practical use because it allows to calculate the Lie algebra of a subgroup of $G$ as a subalgebra of the Lie algebra of $G$.

Proposition 1.21. Let $H$ be a Lie subgroup of the Lie group $G$ and let $\mathfrak{h} \subset \mathfrak{g}$ be the corresponding Lie algebras. Fix $X \in \mathfrak{g}$. If $X \in \mathfrak{h}$, then $\exp t X \in H$ for every $t \in \mathbb{R}$. Conversely, if $\exp t X \in H$ for every $t \in \mathbb{R}$, then $X \in \mathfrak{h}$.

Refining the above result one obtains the next, which is useful when dealing with the classical matrix groups and algebras.

Proposition 1.22. Let $A$ be an abstract subgroup of the Lie group $G$ and let $\mathfrak{a}$ be a vector subspace of the Lie algebra $\mathfrak{g}$ of $G$. Let $U$ be a neighborhood of $0 \in \mathfrak{g}$ diffeomorphic via exp to the neighborood $V$ of $e \in G$. Suppose that

$$
\exp (U \cap \mathfrak{a})=A \cap V .
$$


Then, endowed with the relative topology, $A$ is a Lie subgroup of $G$ and $\mathfrak{a}$ is its Lie algebra.

The set of all $n \times n$ real matrices endowed with the bracket $[A, B]=A B-B A$ is a Lie algebra, and, as any vector space, a smooth manifold with coordinates given by any choice of a basis. As we have seen in Proposition1.14, the Lie algebra of $G L(d, \mathbb{R})$ is canonically identified with $\mathfrak{g l}(d, \mathbb{R})$. The ordinary matrix exponentiation gives rise to a unique homomorphism from $\mathbb{R}$ into $G L(d, \mathbb{R})$

$$
t \mapsto e^{t A}=\sum_{k=0}^{+\infty} \frac{(t A)^{k}}{k !}
$$

that satisfies the properties (1.7) which define the exponential mapping, so that

$$
\exp A=e^{A}
$$

A classic application of Jordan normal forms yields

$$
\operatorname{det} e^{A}=e^{\operatorname{tr} A}
$$

so that the exponential of any square matrix $A$ is an invertible matrix and exp maps indeed $\mathfrak{g l}(d, \mathbb{R})$ to $G L(d, \mathbb{R})$. We observe en passant that (1.19) implies that the exponential maps $\mathfrak{s l}(d, \mathbb{R})$ to $S L(d, \mathbb{R})$, that is, the Lie algebra of traceless matrices to the Lie group of matrices with determinant equal to one. For later use, we remark that (1.17) entails

$$
{ }^{t}\left(e^{A}\right)=e^{t} A
$$

and that for any invertible $B$

$$
B e^{A} B^{-1}=e^{B A B^{-1}} .
$$

Example 1.23. We show that the Lie algebra of $S p(d, \mathbb{R})$ is

$$
\mathfrak{s p}(d, \mathbb{R})=\left\{X \in \mathfrak{g l}(d, \mathbb{R}):{ }^{t} X J+X J=0\right\},
$$

where $J$ is the canonical skew-symmetric matrix defined in (1.2). If $X \in \mathfrak{s p}(d, \mathbb{R})$, the relation ${ }^{t} X J=-J X$, together with $J^{2}=-I$ and (1.20) and (1.21), implies

$$
{ }^{t}\left(e^{X}\right) J\left(e^{X}\right)=e^{t} X J e^{X}=J\left(J e^{t} X J^{-1}\right) e^{X}=J e^{-J^{t} X J} e^{X}=J e^{-X} e^{X}=J .
$$

Conversely, we show that if $Y=e^{X} \in V \cap S p(d, \mathbb{R})$, then ${ }^{t} X J+J X=0$. This can be done by observing that

$$
J={ }^{t}\left(e^{X}\right) J\left(e^{X}\right)=e^{t} X e^{J X J^{-1}} J
$$

implies $e^{t} X e^{J X J^{-1}}=I$ and hence by (1.12)

$$
e^{-{ }^{t} X}=e^{J X J^{-1}}
$$

Take now a neighborhood $U$ of $0 \in \mathfrak{g l}(d, \mathbb{R})$ diffeomorphic under exp to the neighborhood $V$ of $I \in G L(d, \mathbb{R})$. Assuming that $U$ is small enough, that is, intersecting it with $-{ }^{t} U=\left\{-{ }^{t} Z: Z \in U\right\}$ and with $J U J^{-1}=\left\{J Z J^{-1}: Z \in U\right\}$, which are both open and contain the zero matrix, we may assume that both $-{ }^{t} X$ and $J X J^{-1}$ belong to $U$, where the exponential is a diffeomorphism. Hence $-{ }^{t} X=J X J^{-1}$, which amounts 
to ${ }^{t} X J+J X=0$. Applying now Proposition 1.22 we obtain the claimed description of $\mathfrak{s p}(d, \mathbb{R})$, which can be made even more explicit. Indeed, if

$$
X=\left[\begin{array}{cc}
A & B \\
C & D
\end{array}\right] \in \mathfrak{s p}(d, \mathbb{R})
$$

then

$$
0={ }^{t} X J+J X=\left[\begin{array}{ll}
-{ }^{t} C+C & { }^{t} A+D \\
-{ }^{t} D-A & { }^{t} B-B
\end{array}\right]
$$

implies that in fact $X \in \mathfrak{s p}(d, \mathbb{R})$ if and only if

$$
X=\left[\begin{array}{cc}
A & B \\
C & -{ }^{t} A
\end{array}\right], \quad B, C \in \operatorname{Sym}(d, \mathbb{R}) .
$$

Example 1.24. Arguing as in the previous example, one can show that the Lie algebra of the special orthogonal group, namely the compact Lie group

$$
S O(d)=\left\{g \in G L(d, \mathbb{R}):{ }^{t} g g=I, \operatorname{det} g=1\right\},
$$

is the Lie algebra of skew-symmetric matrices

$$
\mathfrak{s o}(d)=\left\{X \in \mathfrak{g l}(d, \mathbb{R}):{ }^{t} X+X=0\right\} .
$$

Example 1.25. Another basic fact: the Lie algebra of the unitary group

$$
U(n)=\left\{g \in G L(n, \mathbb{C}):{ }^{t} \bar{g} g=I\right\}
$$

is the Lie algebra of skew-hermitian matrices

$$
\mathfrak{u}(d)=\left\{X \in \mathfrak{g l}(d, \mathbb{C}):{ }^{t} \bar{X}+X=0\right\} .
$$

1.2.5. Closed subgroups. As the examples at the end of the previous section indicate, many of the most interesting matrix Lie groups arise by imposing extra equations on $G L(d, \mathbb{R})$. Since these equations are often of the form $F\left(g_{i j}\right)=0$ with $F: \mathbb{R}^{n^{2}} \rightarrow \mathbb{R}$ a polynomial or a rational function of the entries, hence continuous on some open set, their solutions cut out subgroups that are topologically closed. The closed subgroups are very special. The first major result is the followimng

Theorem 1.26. (Cartan) Let $G$ be a Lie group and let $A$ be a closed subgroup of $G$. Then $A$ has a unique smooth (in fact analytic) structure that makes it a Lie subgroup of $G$.

Theorem 1.27. Let $G$ be a connected Lie group with Lie algebra $\mathfrak{g}$ and let $\varphi: G \rightarrow H$ be a Lie group homomorphism of $G$ into the Lie group $H$, whose Lie algebra is $\mathfrak{h}$. Then:

(i) $\operatorname{ker}(\varphi)$ is a closed Lie subgroup of $G$ with Lie algebra $\operatorname{ker}(d \varphi)$;

(ii) $\varphi(G)$ is a Lie subgroup of $H$ with Lie algebra $d \varphi(\mathfrak{g}) \in \mathfrak{h}$.

An important example of closed Lie subgroup is the center $Z_{G}$ of $G$, namely

$$
Z_{G}=\left\{g \in G: g x g^{-1}=x, \quad \text { for all } x \in G\right\} .
$$

The fact that $Z_{G}$ is indeed closed can either be shown directly (with sequences) or by using Corollary 1.30 below, which exhibits the center as the kernel of a very important 
homomorphism of $G$ into the automorphisms of its Lie algebra, the adjoint representation. Observe that, besides being closed, the center $Z_{G}$ is always normal in $G$, just as any other kernel of any smooth homomorphism.

Suppose now that $H$ is any closed and normal subgroup of $G$, not necessarily its center. Then $G / H$ has a natural group structure, and it is natural to ask whether it might be a Lie group. This is indeed the case:

Theorem 1.28. If $H$ is a closed and normal subgroup of $G$ then there exists a unique manifold structure on the quotient group $G / H$ that turns it into a Lie group. Moreover, the natural projection $p: G \rightarrow G / H$ is a smooth surjection.

The projection map $p: G \rightarrow G / H$ described in the above proposition is thus a smooth Lie group homomorphism whose kernel is exactly $H$. Therefore, the closed normal subgroups always do appear as kernels of smooth homomorphisms.

Many examples of quotient Lie groups can be given. In Chapter 2 below we are mostly concerned with the Heisenberg group $\mathbb{H}^{d}$, whose center is isomorphic to $\mathbb{R}$. The quotient $\mathbb{H}^{d} / Z$ is (isomorphic to) the Abelian group $\mathbb{R}^{2 d}$.

1.2.6. Adjoint representations. The most important finite dimensional representation of a Lie group $G$ is certainly the adjoint representation, which acts on its Lie algebra $\mathfrak{g}$. Given any real Lie algebra $\mathfrak{g}$, we shall denote by $\mathfrak{g l}(\mathfrak{g})$ the Lie algebra of all endomorphisms of $\mathfrak{g}$ with the commutator as bracket and by $G L(\mathfrak{g})$ the group of all non singular endomorphisms of $\mathfrak{g}$ as a vector space. Hence $\mathfrak{g l}(\mathfrak{g})$ is the Lie algebra of $G L(\mathfrak{g})$. The map $X \mapsto \operatorname{ad} X$ is a Lie algebra homomorphism whose image is a Lie subalgebra of $\mathfrak{g l}(\mathfrak{g})$ denoted ad $\mathfrak{g}$. Let $\operatorname{Int}(\mathfrak{g})$ be the connected Lie subgroup of $G L(\mathfrak{g})$ whose Lie algebra is ad $\mathfrak{g}$. The group $\operatorname{Int}(\mathfrak{g})$ is called the adjoint group of $\mathfrak{g}$. Schematically:

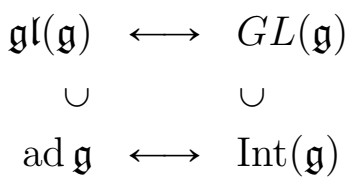

where the arrows stand for the correspondence group-algebra. Next, let $\operatorname{Aut}(\mathfrak{g})$ be the Lie subgroup of $G L(\mathfrak{g})$ consisting of all the automorphisms of $\mathfrak{g}$ (the invertible Lie algebra homomorphisms of $\mathfrak{g}$ onto itself) and denote by $\partial \mathfrak{g}$ its Lie algebra. We know that $\partial \mathfrak{g}$ consits of all the endomorphisms $D \in \mathfrak{g l}(\mathfrak{g})$ such that $\exp t D \in \operatorname{Aut}(\mathfrak{g})$ for every $t \in \mathbb{R}$. From $\exp (t D)[X, Y]=[\exp (t D) X$, $\exp (t D) Y]$, taking the derivative at $t=0$, it follows that

$$
D[X, Y]=[D X, Y]+[X, D Y]
$$

Any such operator is called a derivation of $\mathfrak{g}$. Conversely, if $D$ is a derivation, then by induction

$$
D^{k}[X, Y]=\sum_{i+j=k} \frac{k !}{i ! j !}\left[D^{i} X, D^{j} Y\right],
$$

so that $\exp (t D)[X, Y]=[\exp (t D) X, \exp (t D) Y]$. It follows that $\partial \mathfrak{g}$ consists of all the derivations of $\mathfrak{g}$. Finally, since $\operatorname{ad} X$ is a derivation of $\mathfrak{g}$ for every $X \in \mathfrak{g}$, we may 
refine the preceeding diagram and get:

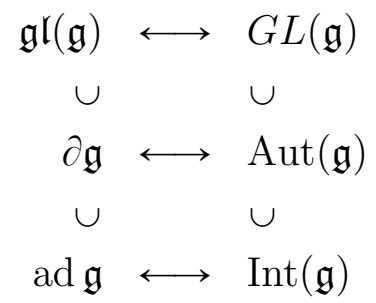

It is easy to see that $\operatorname{Int}(\mathfrak{g})$ is a normal subgroup of $\operatorname{Aut}(\mathfrak{g})$ and ad $\mathfrak{g}$ is an ideal in $\partial \mathfrak{g}$.

Let $G$ be a Lie group and take $g \in G$. Denote by $i_{g}$ the inner conjugation in $G$, namely $x \mapsto g x g^{-1}$ and put

$$
\operatorname{Ad} g:=d i_{g}: \mathfrak{g} \rightarrow \mathfrak{g} .
$$

Since $i_{g}$ is an isomorphism go $G$ it follows that $\operatorname{Ad} g \in \operatorname{Aut}(\mathfrak{g})$. The map

$$
\operatorname{Ad}: G \rightarrow \operatorname{Aut}(\mathfrak{g}), \quad g \mapsto \operatorname{Ad} g
$$

is a homomorphism of Lie groups and is called the adjoint representation of $G$. As it will be clear in the sections that follow, Ad is indeed a finite dimensional representation of $G$ (on a real vector space). On the classical matrix Lie groups, in particular on $G L(d, \mathbb{R})$ and hence on its closed subgroups, we have

$$
\operatorname{Ad} g(X)=g X g^{-1} \text {. }
$$

Also, the universal intertwining property of exp given in Theorem 1.20 entails:

$$
\exp (\operatorname{Ad} g(X))=\exp \left(d i_{g}(X)\right)=i_{g}(\exp X)=g(\exp X) g^{-1},
$$

which is a general version of (1.21).

Theorem 1.29. Ad is a smooth map and $d \mathrm{Ad}=\mathrm{ad}$. In particular, for any $X \in \mathfrak{g}$

$$
\operatorname{Ad}(\exp X)=e^{\operatorname{ad} X}
$$

Corollary 1.30. The adjoint representation of $G$ is a smooth surjective homomorphism of $G$ onto $\operatorname{Int}(\mathfrak{g})$ whose kernel is the center $Z_{G}$ of $G$. Hence, $G / Z_{G} \simeq \operatorname{Int}(\mathfrak{g})$

From the previous result and from Theorem 1.2.5, it follows that the center of a connected Lie group is a closed Lie subgroup. It then follows again from Theorem 1.2.5 that the Lie algebra of the center of $G$ is the center of the Lie algebra, namely

$$
\mathfrak{z}_{\mathfrak{g}}=\{X \in \mathfrak{g}:[X, Y]=0 \text { for all } Y \in \mathfrak{g}\} .
$$

We deduce from this that a Lie group is Abelian (i.e., it has trivial center) if and only if its Lie algebra is such. 
1.2.7. Semidirect products. Suppose that $G$ and $H$ are two Lie groups and that there we are given a group homomorphism

$$
\tau: H \rightarrow \operatorname{Aut}(G), \quad h \mapsto \tau_{h}
$$

such that the map $(g, h) \mapsto \tau_{h}(g)$ is a smooth map of $G \times H$ into $H$. Hence, for every $h \in H$ the map $\tau_{h}$ is an invertible Lie group homomorphism of $G$ onto itself, and $\tau_{h k}=\tau_{h} \circ \tau_{k}$ for every $h, k \in H$. It is then possible to define the semidirect product of $G$ and $H$. It is the group denoted $G \rtimes H$ whose elements are those of $G \times H$ and where the product is defined by

$$
\left(g_{1}, h_{1}\right)\left(g_{2}, h_{2}\right)=\left(g_{1} \tau_{h_{1}}\left(g_{2}\right), h_{1} h_{2}\right) .
$$

It is immediate to check that this is a group law, and indeed smooth, so that $G \times H$ is a Lie group. Inverses are given by

$$
(g, h)^{-1}=\left(\tau_{h^{-1}}\left(g^{-1}\right), h^{-1}\right) .
$$

If we identify $G$ and $H$ with the subsets of $G \rtimes H$ given by $\{(g, e): g \in G\}$ and $\{(e, h): h \in H\}$, respectively, then both $G$ and $H$ are closed subgroups and $G$ is a normal subgroup in $G \rtimes H$.

Example 1.31. The most obvious example of semidirect product is the " $a x+b$ " group. Evidently, $H=\mathbb{R}_{+}, G=\mathbb{R}$ and $\tau_{a}(b)=a b$. One possible generalization in higher dimensions is the Euclidean motion group in $\mathbb{R}^{d}$, where $H=S O(d)$ acts in the natural linear fashion on $G=\mathbb{R}^{d}$.

Example 1.32. Another example of semidirect product is the Poincaré group. Here

$$
H=S O(1,3)=\left\{h \in S L(4, \mathbb{R}):{ }^{t} h I_{1,3} h=I_{1,3}\right\},
$$

where

$$
I_{1,3}=\left[\begin{array}{cccc}
-1 & 0 & 0 & 0 \\
0 & 1 & 0 & 0 \\
0 & 0 & 1 & 0 \\
0 & 0 & 0 & 1
\end{array}\right]
$$

As $G$, we take $\mathbb{R}^{4}$. Again, $\tau_{h}(x)=h x$ for $h \in S O(1,3)$ and $x \in \mathbb{R}^{4}$.

Other useful examples of semidirect products will be discussed in Section 2 and also in Section 3.

Exercise 1.33. This exercise aims at showing what is the sensible notion of semidirect product of Lie algebras. Let $\mathfrak{a}$ and $\mathfrak{b}$ denote two Lie algebras and suppose that we are given a Lie algebra homomorphism $\pi: \mathfrak{a} \rightarrow \partial \mathfrak{b}$ into the derivations of $\mathfrak{b}$. Show that there exists a unique Lie algebra structure on the vector space $\mathfrak{g}=\mathfrak{a}+\mathfrak{b}$ which preserves the Lie algebra structures of both $\mathfrak{a}$ and $\mathfrak{b}$, and such that $[A, B]=\pi(A)(B)$ for every $A \in \mathfrak{a}$ and every $B \in \mathfrak{b}$. Further, show that $\mathfrak{a}$ is a subalgebra and $\mathfrak{b}$ is an ideal in $\mathfrak{g}$. 
1.2.8. Haar measure on Lie groups and integration. For Lie groups, left Haar measures are very easy to construct. One takes any positive definite inner product on the tangent space at the identity $T_{e} G$ and carries it around with the differential of left translations, thereby obtainining a Riemannian structure. The corresponding volume form is a Haar measure. Furthermore, in every local coordinate system, it is given by a $C^{\infty}$ density times the Lebesgue measure. We do not appeal here to these facts that go beyond our scopes, and simply quote a handy result.

Proposition 1.34. If $G$ is a Lie group whose underlying manifold is an open set in $\mathbb{R}^{d}$ and if the left translations are given by affine maps, that is

$$
x y=A(x) y+b(x),
$$

where $A(x)$ is a linear transformation and $b(x) \in \mathbb{R}^{d}$, then $|\operatorname{det} A(x)|^{-1} d x$ is a Haar measure on $G$.

Example 1.35. For example, in the group " $a x+b$ " the left translations are

$$
l_{(a, b)}(\alpha, \beta)=\left[\begin{array}{ll}
a & 0 \\
0 & a
\end{array}\right]\left[\begin{array}{l}
\alpha \\
\beta
\end{array}\right]+\left[\begin{array}{l}
0 \\
b
\end{array}\right]
$$

so that by Proposition 1.34 we have

$$
|\operatorname{det} A(a, b)|^{-1} d a d b=\frac{d a}{a^{2}} d b .
$$

As for the modular function, in any Lie group we have

$$
\Delta(g)=|\operatorname{det} \operatorname{Ad}(g)|^{-1} .
$$

It is possible to realize the " $a x+b$ " group as a matrix group. The reader may check that the correspondence

$$
(a, b) \leftrightarrow\left[\begin{array}{ll}
a & b \\
0 & 1
\end{array}\right]
$$

establisehes an isomorphism of " $a x+b$ " with a closed Lie subgroup of $G L(2, \mathbb{R})$, whose Lie algebra ie easily seen to consists of the matrices

$$
\left[\begin{array}{cc}
A & B \\
0 & 0
\end{array}\right]
$$

The adjoint representation takes the form

$$
\left[\begin{array}{ll}
a & b \\
0 & 1
\end{array}\right]\left[\begin{array}{cc}
A & B \\
0 & 0
\end{array}\right]\left[\begin{array}{cc}
a^{-1} & -b a^{-1} \\
0 & 1
\end{array}\right]=\left[\begin{array}{cc}
A & -b A+a B \\
0 & 0
\end{array}\right]
$$

and it is thus the linear map

$$
\left[\begin{array}{l}
A \\
B
\end{array}\right] \mapsto\left[\begin{array}{cc}
1 & 0 \\
-b & a
\end{array}\right]\left[\begin{array}{l}
A \\
B
\end{array}\right]
$$

It follows that

$$
\Delta(a, b)=\left|\operatorname{det}\left[\begin{array}{cc}
1 & 0 \\
-b & a
\end{array}\right]\right|^{-1}=a^{-1}
$$


One can check directly with a change of variable that indeed

$$
\int_{\mathbb{R}_{+} \times \mathbb{R}} f((\alpha, \beta)(a, b)) \frac{d a}{a^{2}} d b=\alpha \int_{\mathbb{R}_{+} \times \mathbb{R}} f((a, b)) \frac{d a}{a^{2}} d b .
$$

1.2.9. Convolutions. The convolution of integrable functions defined on $\mathbb{R}^{d}$ involves translations. It is thus suitable for interpretation on any group $G$ on which a reasonable notion of measure is given, like for example Lie groups. Indeed, if $G$ is a Lie group with left Haar measure $d x$ and if $f, g \in L^{1}(G)$, then the convolution of $f$ and $g$ is the function defined by

$$
f * g(x)=\int_{G} f(y) g\left(y^{-1} x\right) d y
$$

By the left invariance of $d x$, we have that

$$
\int_{G} \int_{G}\left|f(y) g\left(y^{-1} x\right)\right| d x d y=\int_{G} \int_{G}|f(y) g(x)| d x d y=\|f\|_{1}\|g\|_{1}
$$

so that, by Fubini's theorem, $f * g \in L^{1}(G)$ and

$$
\|f * g\| \leqslant\|f\|_{1}\|g\|_{1} \text {. }
$$

The convolution of $L^{1}$ functions can be expressed in several different ways:

$$
\begin{aligned}
f * g(x) & =\int_{G} f(y) g\left(y^{-1} x\right) d y \\
& =\int_{G} f(x y) g\left(y^{-1}\right) d y \\
& =\int_{G} f\left(y^{-1}\right) g(y x) \Delta\left(y^{-1}\right) d y \\
& =\int_{G} f\left(x y^{-1}\right) g(y) \Delta\left(y^{-1}\right) d y .
\end{aligned}
$$

It must be noticed that, unless the group $G$ is itself commutative, in general the convolution is not commutative.

Exercise 1.36. Show that on the affine group " $a x+b$ " the convolution is

$$
f * g(\alpha, \beta)=\int_{\mathbb{R}} \int_{\mathbb{R}_{+}} f(a, b) g\left(\frac{\alpha}{a}, \frac{\beta-b}{a}\right) \frac{d a}{a^{2}} d b
$$

and verify that it is not commutative.

Exercise 1.37. Show that on any Lie group $G$ and for any $f, g \in L^{1}(G)$ one has

$$
\lambda(x)(f * g)=(\lambda(x) f) * g, \quad \rho(x)(f * g)=f *(\rho(x) g),
$$

where $\lambda$ and $\rho$ are the left and right translations, respectively.

The mapping properties of (left or right) convolution operators have been studied in much detail. We collect here some facts that will be useful. For a detailed proof of many statements see [4], whereas [14] contains the basic facts. If $f, g \in L_{\text {loc }}^{1}(G)$, if the convolution $f * g$ defined by (1.26) exists, and if $|f| *|g|$ is in $L_{\text {loc }}^{1}(G)$, then we say that $f$ and $g$ are convolvable. 
Theorem 1.38. Let $f, g$ be two measurable funcions on the Lie group $G$.

(i) If $f \in L^{1}(G)$ and $g \in L^{p}(G)$ with $1 \leqslant p \leqslant \infty$, then the integrals in (1.27) converge for almost every $x \in G$, we have $f * g \in L^{p}(G)$ and

$$
\|f * g\|_{p} \leqslant\|f\|_{1}\|g\|_{p} .
$$

Furthermore, if $p=\infty$, then $f * g$ is also continuous.

(ii) If $f \in L^{p}(G), g \in L^{q}(G)$ and $\check{g} \in L^{q}(G)$ where $1<p<+\infty$ and $1<q<+\infty$ satisfy $\frac{1}{p}+\frac{1}{q}=1+\frac{1}{r}$ with $r>1$, then $f$ and $g$ are convolvable and $f * g$ belongs to $L^{r}(G)$. Furthermore, if $\|\check{g}\|_{q}=\|g\|_{q}$, then

$$
\|f * g\|_{r} \leqslant\|f\|_{p}\|g\|_{q} .
$$

(iii) If $f \in L^{p}(G), g \in L^{q}(G)$ and $\check{g} \in L^{q}(G)$, where $1<p<+\infty$ and $\frac{1}{p}+\frac{1}{q}=1$, then $f$ and $g$ are convolvable, $f * g$ belongs to $C_{0}(G)$ and

$$
\|f * g\|_{\infty} \leqslant\|f\|_{p}\|\check{g}\|_{q} .
$$

1.3. Representation theory. Let $\mathcal{H}_{1}$ and $\mathcal{H}_{2}$ be two Hilbert spaces and suppose that $T: \mathcal{H}_{1} \rightarrow \mathcal{H}_{2}$ is linear and bounded, that is $T \in \mathcal{B}\left(\mathcal{H}_{1}, \mathcal{H}_{2}\right)$. Recall that $T$ is an isometry if $\|T u\|=\|u\|$ for every $u \in \mathcal{H}_{1}$. Since $\|T u\|^{2}=\langle T u, T u\rangle=\left\langle T^{*} T u, u\right\rangle$ and $\|u\|^{2}=\langle u, u\rangle$, the polarition identity implies that $T$ is an isometry if and only if $T^{*} T=i d_{\mathcal{H}_{1}}$. Hence, isometries are injective, but they are not necessarily surjective. A bijective isometry is called a unitary map. If $T$ is unitary, such is also $T^{-1}$ and in this case $T T^{*}=\operatorname{id}_{\mathcal{H}_{2}}$. In particular if $\mathcal{H}_{1}=\mathcal{H}_{2}=\mathcal{H}$, the set

$$
\mathcal{U}(\mathcal{H})=\{T \in \mathcal{B}(\mathcal{H}): T \text { is unitary }\}
$$

forms a group. Evidently, $\mathcal{U}(\mathcal{H}) \subset \mathcal{B}(\mathcal{H})$, the space of bounded linear operators of $\mathcal{H}$ onto itself.

Let now $G$ be a $\mathrm{Lie}^{1}$ group.

Definition 1.39. A unitary representation of $G$ on the Hilbert space $\mathcal{H}$ is a group homomorphism $\pi: G \rightarrow \mathcal{U}(\mathcal{H})$ continuous in the strong operator topology. This means:

i) $\pi(g h)=\pi(g) \pi(h)$ for every $g, h \in G$;

ii) $\pi\left(g^{-1}\right)=\pi(g)^{-1}=\pi(g)^{*}$ for every $g \in G$;

iii) $g \mapsto \pi(g) u$ is continuous from $G$ to $\mathcal{H}$, for every $u \in \mathcal{H}$.

Observe that from the equality $\|\pi(g) u-\pi(h) u\|=\left\|\pi\left(h g^{-1}\right) u-u\right\|$ it follows that it is enough to check iii) for $g=e$, the identity of $G$.

Example 1.40. Let $G=\mathbb{R}$ be additive group and $\mathcal{H}=\mathbb{C}$. For every $s \in \mathbb{R}$ we define the function $\chi_{s}(t)=e^{i t s}$ and we identify the complex number $e^{i t s}$ with the

\footnotetext{
${ }^{1}$ In all what follows, it would suffice to consider a locally compact Hausdorff topological group.
} 
multiplication operator on $\mathbb{C}$ defined by $z \mapsto z e^{i t s}$. Clearly, $\chi_{s}: \mathbb{R} \rightarrow \mathcal{U}(\mathbb{C})$ is a unitary representation, because

$$
\begin{aligned}
& \chi_{s}(x+y)=\chi_{s}(x) \chi_{s}(y) \\
& \chi_{s}(-x)=\chi_{s}(x)^{-1}=\overline{\chi_{s}(x)} \\
& t \mapsto e^{i t s} z \text { is continuous for every } z \in \mathbb{C} .
\end{aligned}
$$

Example 1.41. Let $G$ be any locally compact group and choose $\mathcal{H}=L^{2}(G)$. Define

$$
\lambda: G \rightarrow \mathcal{U}(\mathcal{H}), \quad g \mapsto \lambda_{x}, \quad \lambda_{x} f(y)=f\left(x^{-1} y\right)
$$

It is easy to check that this is a unitary representation, the so-called left regular representation. Similarly, the right regular representation is defined by

$$
\rho: G \rightarrow \mathcal{U}(\mathcal{H}), \quad g \mapsto \rho_{x}, \quad \rho_{x} f(y)=\Delta(x)^{1 / 2} f(y x) .
$$

The modular function is necessary in order that $\rho_{x}$ is unitary.

Example 1.42. Let $G$ be the " $a x+b$ " group and $\mathcal{H}=L^{2}(\mathbb{R})$. Define

$$
\pi(a, b) f(x)=\frac{1}{\sqrt{a}} f\left(\frac{x-b}{a}\right), \quad a>0, b \in \mathbb{R}
$$

the so-called wavelet representation. Notice that it is just the composition of the two very important and basic unitary maps

$$
\begin{aligned}
& T_{b} f(x)=f(x-b) \quad \text { (translation operator) } \\
& D_{a} f(x)=\frac{1}{\sqrt{a}} f\left(\frac{x}{a}\right) \quad \text { (dilation operator) }
\end{aligned}
$$

for indeed

$$
T_{b} D_{a} f(x)=T_{b}\left(D_{a} f\right)(x)=D_{a} f(x-b)=\frac{1}{\sqrt{a}} f\left(\frac{x-b}{a}\right) .
$$

Observe that

$$
T_{b} T_{b^{\prime}}=T_{b+b^{\prime}}, \quad D_{a} D_{a^{\prime}}=D_{a a^{\prime}} .
$$

It is important to notice that $T_{b} D_{a} \neq D_{a} T_{b}$. More precisely,

$$
D_{a} T_{b} f(x)=\frac{1}{\sqrt{a}}\left(T_{b} f\right)\left(\frac{x}{a}\right)=\frac{1}{\sqrt{a}} f\left(\frac{x}{a}-b\right)=\frac{1}{\sqrt{a}} f\left(\frac{x-a b}{a}\right)=T_{a b} D_{a} f(x) .
$$

In other words

$$
D_{a} T_{b}=T_{a b} D_{a}
$$

It follows that

$$
\left(T_{\beta} D_{\alpha}\right)\left(T_{b} D_{a}\right)=T_{\beta}\left(D_{\alpha} T_{b}\right) D_{a}=T_{\beta}\left(T_{\alpha b} D_{a}\right) D_{a}=\left(T_{\beta} T_{\alpha b}\right)\left(D_{a} D_{a}\right)=T_{\beta+\alpha b} D_{\alpha a} .
$$

so that $\pi$ is a homomorphism:

$$
\pi(\alpha, \beta) \pi(a, b)=\pi(\alpha a, \beta+\alpha b)=\pi((\alpha, \beta)(a, b)) .
$$

Finally, it is instructive to check the strong continuity, which is left as an exercise. 
Example 1.43. A variant of (1.32) is the analogous that arises by considering the full affine group acting on $L^{2}(\mathbb{R})$ :

$$
\pi_{\text {full }}(a, b) f(x)=\frac{1}{\sqrt{|a|}} f\left(\frac{x-b}{a}\right), \quad a \in \mathbb{R}^{*}, b \in \mathbb{R}
$$

Definition 1.44. Let $\mathcal{M}$ be a closed subspace of the Hilbert space $\mathcal{H}$. We say that $\mathcal{M}$ is an invariant subspace for the unitary representation $\pi$ if $\pi(g) \mathcal{M} \subset \mathcal{M}$ for every $g \in G$. We say that $\pi$ is irreducible if $\mathcal{H}$ does not contain proper non trivial closed invariant subspaces, that is, closed invariant subspaces other than $\mathcal{H}$ and $\{0\}$.

Exercise 1.45. Prove that if $\mathcal{M}$ is an invariant subspace for $\pi$ then such is also $\mathcal{M}^{\perp}$ and that $\pi=\pi_{\mathcal{M}} \oplus \pi_{\mathcal{M}^{\perp}}$, where $\pi_{\mathcal{M}}$ is the restriction of $\pi$ to $\mathcal{M}$ and similarly for $\mathcal{M}^{\perp}$. This means that for every $g \in G$ the linear map $\pi(g)$ is the direct sum of the linear maps $\pi_{\mathcal{M}}$ and $\pi_{\mathcal{M}^{\perp}}$, each acting on the appropriate space.

Definition 1.46. Let $\pi$ be a unitary representation of $G$ on $\mathcal{H}$ eand take $\xi, \eta \in \mathcal{H}$. The function $G \rightarrow \mathbb{C}$ defined by $g \mapsto\langle\xi, \pi(g) \eta\rangle$ is called the coefficient of $\pi$ relative to $(\xi, \eta)$. If $\xi=\eta$, it is called a diagonal coefficient. Notice that all coefficients are continuous functions and $|\langle\xi, \pi(g) \eta\rangle| \leqslant\|\xi\|\|\eta\|$.

Exercise 1.47. Prove that if $\pi_{1}$ and $\pi_{2}$ are equivalent, they have the same coefficients. Conversely, assume that $\pi_{1}$ and $\pi_{2}$ are irreducible and suppose that they have the same non zero diagonal coefficients. Show that $\pi_{1}$ and $\pi_{2}$ are equivalent. [Hint: take $\xi_{1}$ e $\xi_{2}$ such that $\left\langle\xi_{1}, \pi_{1}(g) \xi_{1}\right\rangle=\left\langle\xi_{2}, \pi_{2}(g) \xi_{2}\right\rangle \neq 0$ for all $g \in G$ and define $U$ on the elements of the form $\xi=\sum_{j=1}^{k} \alpha_{j} \pi_{1}\left(x_{j}\right) \xi_{1}$ via the formula $U \xi=\sum_{j=1}^{k} \alpha_{j} \pi_{2}\left(x_{j}\right) \xi_{2}$.]

Proposition 1.48. . The following two conditions for a representation are equivalent

(i) $\pi$ is irreducible

(ii) if $\xi$ and $\eta$ are non zero vectors in $\mathcal{H}$, then the coefficient $\langle\xi, \pi(g) \eta\rangle$ is non zero as a continuous map.

Proof. Suppose that $\pi$ is irreducible and assume by contradiction that we can find two non zero $\eta, \xi \in \mathcal{H}$ for which $\langle\xi, \pi(g) \eta\rangle=0$. The space

$$
\mathcal{M}_{\eta}=\operatorname{cl}\{\pi(g) \eta: g \in G\}
$$

is a closed invariant subspace, it is not the zero space because $\eta \in \mathcal{M}_{\eta}$ and it cannot be $\mathcal{H}$ because $\xi \in \mathcal{M}_{\eta}^{\perp}$. This contradicts the hypothesis that $\pi$ is irreducible. Conversely, if (ii) holds, take any closed invariant subspace $\mathcal{M} \neq\{0\}$ and take a non zero vector $\eta \in \mathcal{M}$, so that $\pi(g) \eta \in \mathcal{M}$ for every $g \in G$. If $\mathcal{M} \neq \mathcal{H}$, then $\mathcal{M}^{\perp} \neq\{0\}$ and therefore there exists a non zero $\xi \in \mathcal{M}^{\perp}$. But this entails that $\langle\xi, \pi(g) \eta\rangle=0$ for every $g \in G$, contrary to assumption. Hence $\mathcal{M}=\mathcal{H}$ and $\pi$ is irreducible.

Example 1.49. CITARE HEIL WALNUT Using the previous proposition, we show that the wavelet representation (1.32) of the " $a x+b$ " group is not irreducible, whereas the wavelet representation (1.35) of the full affine group is. The calculations that follow are very basic and important. We shall use the Fourier transform $\mathcal{F}$, defined on $L^{1}\left(\mathbb{R}^{d}\right) \cap L^{2}\left(\mathbb{R}^{d}\right)$ by

$$
\hat{f}(\xi)=\mathcal{F} f(\xi)=\int_{\mathbb{R}^{d}} f(x) e^{-2 \pi i x \cdot \xi} d x .
$$


A straightforward computation yields

$$
\begin{gathered}
\mathcal{F}(\pi(a, b) f)(\xi)=\sqrt{a} e^{-2 \pi i b \xi} \hat{f}(a \xi), \quad a>0, b \in \mathbb{R} \\
\mathcal{F}\left(\pi_{\text {full }}(a, b) f\right)(\xi)=\sqrt{|a|} e^{-2 \pi i b \xi} \hat{f}(a \xi), \quad a \in \mathbb{R}^{*}, b \in \mathbb{R} .
\end{gathered}
$$

We start with $\pi$ and show that it is not irreducible. To this end, take two non zero $f, g \in L^{2}(\mathbb{R})$. Then, by Plancherel

$$
\begin{aligned}
\int_{G}|\langle\pi(a, b) f, g\rangle|^{2} \frac{d a d b}{a^{2}} & =\int_{G}|\langle\mathcal{F}(\pi(a, b) f), \mathcal{F} g\rangle|^{2} \frac{d a d b}{a^{2}} \\
& =\int_{G}\left|\int_{\mathbb{R}} \sqrt{a} e^{-2 \pi i b \xi} \hat{f}(a \xi) \overline{\hat{g}(\xi)} d \xi\right|^{2} \frac{d a d b}{a^{2}} \\
& =\int_{G}\left|\left(\mathcal{F}^{-1} \omega_{a}\right)(-b)\right|^{2} d b \frac{d a}{a},
\end{aligned}
$$

where $\omega_{a}(\xi)=\hat{f}(a \xi) \overline{\hat{g}(\xi)}$. Hence, again by Plancherel

$$
\begin{aligned}
\int_{G}|\langle\pi(a, b) f, g\rangle|^{2} \frac{d a d b}{a^{2}} & =\int_{G}\left|\omega_{a}(\xi)\right|^{2} d \xi \frac{d a}{a} \\
& =\int_{\mathbb{R}}\left(\int_{0}^{+\infty}|\hat{f}(a \xi)|^{2} \frac{d a}{a}\right)|\hat{g}(\xi)|^{2} d \xi
\end{aligned}
$$

Define now the following (Hardy) spaces:

$$
\begin{aligned}
& \mathcal{H}_{+}(\mathbb{R})=\left\{f \in L^{2}(\mathbb{R}): \hat{f}(\xi)=0 \text { if } \xi<0\right\} \\
& \mathcal{H}_{-}(\mathbb{R})=\left\{f \in L^{2}(\mathbb{R}): \hat{f}(\xi)=0 \text { if } \xi>0\right\} .
\end{aligned}
$$

Now, if we take $f \in \mathcal{H}_{+}(\mathbb{R})$ and $g \in \mathcal{H}_{-}(\mathbb{R})$, then the support of $\hat{g}$ is contained in the negative reals, so that we may suppose that, in the inner integral in (1.39), $a \xi<0$ for every $a>0$. Hence $a \xi$ is outside the support of $\hat{f}$ for every $a>0$. It follows that the coefficient $\langle\pi(a, b) f, g\rangle$ vanishes and that $\pi$ is not irreducible.

It is actually not hard to show that the Hardy spaces are both closed subspaces of $L^{2}(\mathbb{R})$ and, by (1.37), that they are both invariant under $\pi$. This provides a direct alternative way to see that $\pi$ is not irreducible. The above computations, however, reveal a lot more than the simple fact that $\pi$ is not irreducible. First of all, if we restrict $\pi$ to $\mathcal{H}_{+}(\mathbb{R})$, that is, if both $f, g \in \mathcal{H}_{+}(\mathbb{R})$, then since their Fourier transforms are supported in the positive reals, for any fixed $\xi>0$ we may make the change of variable $a \mapsto a / \xi$ in the inner integral in (1.39) and obtain

$$
\int_{G}|\langle\pi(a, b) f, g\rangle|^{2} \frac{d a d b}{a^{2}}=\left(\int_{0}^{+\infty}|\hat{f}(a)|^{2} \frac{d a}{a}\right)\left(\int_{\mathbb{R}}|\hat{g}(\xi)|^{2} d \xi\right) .
$$

This proves that for $f, g \in \mathcal{H}_{+}(\mathbb{R})$ both non zero we have $\langle\pi(a, b) f, g\rangle \neq 0$ as a continuous function, because neither $\hat{f}$ nor $\hat{g}$ can identically vanish. Hence the restriction of $\pi$ to $\mathcal{H}_{+}(\mathbb{R})$ is irreducible. The same holds true for $\mathcal{H}_{-}(\mathbb{R})$. We leave it as an exercise 
to show that $L^{2}(\mathbb{R})=\mathcal{H}_{+}(\mathbb{R}) \oplus \mathcal{H}_{-}(\mathbb{R})$ and that $\pi=\pi_{+} \oplus \pi_{-}$. We observe en passant that if $f \in \mathcal{H}_{+}(\mathbb{R})$ is such that

$$
\int_{0}^{+\infty}|\hat{f}(a)|^{2} \frac{d a}{a}=1
$$

then, by Plancherel and (1.42) we have

$$
\|\langle\pi(a, b) f, g\rangle\|_{L^{2}(G)}=\|g\|_{\mathcal{H}_{+}(\mathbb{R})}
$$

and similarly for $\mathcal{H}_{-}(\mathbb{R})$. Equation (1.43) is called a Calderón equation and a function $f \in \mathcal{H}_{+}(\mathbb{R})$ that satisfies it is called a wavelet.

Let us now consider the full affine group. A calculation analogous to the previous one yields

$$
\int_{G_{\text {full }}}\left|\left\langle\pi_{\text {full }}(a, b) f, g\right\rangle\right|^{2} \frac{d a d b}{a^{2}}=\int_{\mathbb{R}}\left(\int_{\mathbb{R}^{*}}|\hat{f}(a \xi)|^{2} \frac{d a}{|a|}\right)|\hat{g}(\xi)|^{2} d \xi
$$

This time, for any non zero $\xi$, as $a$ ranges in $\mathbb{R}$ the numbers $a \xi$ cover $\mathbb{R}$ and the change of variable $a \mapsto a / \xi$ in the inner integral gives

$$
\int_{G_{\text {full }}}\left|\left\langle\pi_{\text {full }}(a, b) f, g\right\rangle\right|^{2} \frac{d a d b}{a^{2}}=\left(\int_{\mathbb{R}^{*}}|\hat{f}(a)|^{2} \frac{d a}{|a|}\right)\left(\int_{\mathbb{R}}|\hat{g}(\xi)|^{2} d \xi\right),
$$

which cannot be zero if both $f$ and $g$ are not zero. This proves that $\pi_{\text {full }}$ is an irreducible unitary representation of $G_{\text {full }}$. The Calderón equation for the full affine group is thus

$$
\int_{\mathbb{R}}|\hat{f}(a)|^{2} \frac{d a}{|a|}=1
$$

Definition 1.50. Let $\pi$ be a representation of $G$ on $\mathcal{H}$. A vector $u \in \mathcal{H}$ is called a cyclic vector for the representation if the closed linear span $\mathcal{M}_{u}$ of $\{\pi(x) u: x \in G\}$ coincides with $\mathcal{H}$. Clearly, in general, $\mathcal{M}_{u}$ is a closed $\pi$-invariant subspace of $\mathcal{H}$. The representation is called cyclic if it has a cyclic vector.

Definition 1.51. Let $\pi_{i}: G \rightarrow \mathcal{U}\left(\mathcal{H}_{i}\right), i=1,2$ be two unitary representations of $G$. They are called unitarily equivalent if there exists a unitary operator $U: \mathcal{H}_{1} \rightarrow \mathcal{H}_{2}$ such that

$$
\pi_{2}(g) \circ U=U \circ \pi_{1}(g), \quad \text { for every } g \in G .
$$

In this case, $U$ is called an intertwining operator between $\pi_{1}$ and $\pi_{2}$. The set of all intertwining operators between $\pi_{1}$ and $\pi_{2}$ will be denoted $\mathcal{I}\left(\pi_{1}, \pi_{2}\right)$. If $\pi_{1}=\pi_{2}=\pi$, we write $\mathcal{I}(\pi, \pi)=\mathcal{I}(\pi)$.

Exercise 1.52. controllare se dopo rimane lambda o L Let $\lambda$ be the left regular representation of $\mathbb{R}$ on $L^{2}(\mathbb{R})$, namely $\lambda_{x} f(y)=f(y-x)$, and let $\rho$ the right regular representation of $\mathbb{R}$ on $L^{2}(\mathbb{R})$, namely $\rho_{x} f(y)=f(y+x)$. Exhibit a unitary operator on $L^{2}(\mathbb{R})$ that intertwines $\rho$ and $\lambda$.

Exercise 1.53. Let $\mathcal{M} \subset \mathcal{H}$ be a closed subspace and denote by $P$ the orthogonal projection onto $\mathcal{M}$. Prove that $\mathcal{M}$ is $\pi$-invariant if and only if $P \in \mathcal{I}(\pi)$. 
The next result is of crucial importance in representation theory. For a proof see for example [15].

Lemma 1.54. (Schur's lemma.) i) A unitary representation of $G$ is irreducibile if and only if $\mathcal{I}(\pi)$ contains only scalar multiples of the identity.

ii) Let $\pi_{1}$ e $\pi_{2}$ be two unitary irreducible representations of $G$. If they are equivalent, then $\mathcal{I}\left(\pi_{1}, \pi_{2}\right)$ has dimension one, otherwise $\mathcal{I}\left(\pi_{1}, \pi_{2}\right)=\{0\}$.

Corollary 1.55. Every irreducible representation of an Abelian group is one dimensional.

Proof. Suppose that $G$ is Abelian and take a representation $\pi$ of $G$. Then all the operators $\pi(x)$ commute and hence are in $\mathcal{I}(\pi)$. If $\pi$ is irreducible, then $\pi(x)$ is a constant multiple of the identity and every one dimensional subspace of $\mathcal{H}_{\pi}$ is invariant. Therefore $\mathcal{H}_{\pi}$ must be one dimensional.

Suppose that $G$ is a Lie group with a unitary representation $\pi$ and that $N$ is a closed and normal subgroup of $G$. Then, as we know from Theorem 1.28, the quotient $G / N$ is a Lie group. If the kernel of $\pi$ contains $N$, that is, if $\pi(n)$ is the identity operator for every $n \in N$, then it is possible to project $\pi$ to a representation $\tilde{\pi}$ of the quotient $G / N$. Indeed, one puts

$$
\tilde{\pi}(g N)=\pi(g)
$$

and obtains a well defined unitary representation of the Lie group $G / N$ on the same Hilbert space on which $\pi$ was defined. It is easy t see that if $\pi$ is irreducible, then such is $\tilde{\pi}$

1.4. Square integrability. We are interested in the properties of the coefficients of a given unitary representation of $G$. More precisely, we fix a vector $u \in \mathcal{H}$ and consider the so-called voice transform associated to it, namely

$$
V_{u}: \mathcal{H} \rightarrow L^{\infty}(G) \cap C(G)
$$

defined by

$$
V_{u} v(x)=\langle v, \pi(x) u\rangle, \quad v \in \mathcal{H} .
$$

Thus, for fixed $u$, the voice transform maps elements in the Hilbert space $\mathcal{H}$ to functions on $G$ that are bounded and continuous, as established formally in Proposition 1.56 below. For reasons that will become clear in what follows, the function

$$
K_{u}(x)=V_{u} u(x)=\langle u, \pi(x) u\rangle
$$

is of particular relevance, and is called the kernel of the voice transform. By definition, it is the diagonal coefficient of the representation corresponding to $u$. Below and in the remaining part of this article we write

$$
\breve{f}(x)=f\left(x^{-1}\right)
$$

whenever $f$ is a function on the group $G$. 
Proposition 1.56. Let $\pi$ be a unitary representation of the Lie group $G$ on the Hilbert space $\mathcal{H}$ and let $u \in \mathcal{H}$ be a fixed vector. Then:

(i) the transforms $V_{u} v$ are bounded continuous functions on $G$, for all $v \in \mathcal{H}$;

(ii) the voice transform $V_{u}$ satisfies $V_{u} \circ \pi(x)=\lambda(x) \circ V_{u}$ for every $x \in G$;

(iii) $u$ is a cyclic vector for $\pi$ if and only if $V_{u}$ is an injective map of $\mathcal{H}$ into $L^{\infty}(G) \cap C(G)$;

(iv) the kernel $K_{u}$ satisfies $\overline{K_{u}}=\widetilde{K_{u}}$.

Proof. (i) The continuity of $x \mapsto\langle v, \pi(x) u\rangle$ follows from the continuity of the representation (strong continuity implies weak continuity). Boundedness follows from

$$
\left|V_{u} v(x)\right|=|\langle v, \pi(x) u\rangle| \leqslant\|v\|\|u\| .
$$

(ii) This is just a direct computation that uses the fact that $\pi$ is unitary, hence $\pi(y)^{*}=\pi\left(y^{-1}\right)$. Indeed:

$$
V_{u}(\pi(y) v)(x)=\langle\pi(y) v, \pi(x) u\rangle=\left\langle v, \pi\left(y^{-1} x\right) u\right\rangle=V_{u} v\left(y^{-1} x\right)=\left(\lambda(y) V_{u} v\right)(x)
$$

(iii) Take $v \in \mathcal{H}$. Then

$$
V_{u} v(\cdot)=0 \Longleftrightarrow\langle v, \pi(\cdot) u\rangle=0 \Longleftrightarrow v \in \mathcal{M}_{u}^{\perp},
$$

where the first two equalities refer to the function on $G$ which is identically zero. Now, $u$ is cyclic if and only if $\mathcal{M}_{u}^{\perp}=\{0\}$ and this is equivalent to the fact that the only zero transform $V_{u} v$ is when $v=0$, which is the injectivity of $V_{u}$.

(iv) This is immediate, since

$$
\overline{K_{u}(x)}=\overline{\langle u, \pi(x) u\rangle}=\langle\pi(x) u, u\rangle=\left\langle u, \pi\left(x^{-1}\right) u\right\rangle=K_{u}\left(x^{-1}\right)=\widetilde{K_{u}}(x) .
$$

Definition 1.57. Let $\pi$ be a unitary representation of the Lie group $G$ on the Hilbert space $\mathcal{H}$. If there exists a vector $u \in \mathcal{H}$, called admissible, for which the corresponding voice transform takes values in $L^{2}(G)$ and is an isometry, that is, if

$$
V_{u}: \mathcal{H} \rightarrow L^{2}(G), \quad\left\|V_{u} v\right\|=\|v\|
$$

for every $v \in \mathcal{H}$, then we say that the system $(G, \pi, \mathcal{H}, u)$ is reproducing, or, for short, that $u$ is an admissible vector for $\pi$.

By the polarization identity, the isometry property $\left\|V_{u} v\right\|=\|v\|$ is equivalent to

$$
\left\langle V_{u} v, V_{u} w\right\rangle_{2}=\langle v, w\rangle_{\mathcal{H}}, \quad v, w \in \mathcal{H},
$$

where we have stressed that the first inner product is in $L^{2}(G)$ and the second in $\mathcal{H}$. We observe en passant that if $u$ is an admissible vector for $\pi$, then it is a cyclic vector for $\pi$. This is because if $V_{u}$ is an isometry, then it is injective on $\mathcal{H}$ and (iii) of Proposition 1.56 applies.

In the literature, the notion of reproducing system is primarily studied when $\pi$ is irreducible. If this is the case, and if $\pi$ admits an admissible vector, then one says that $\pi$ is square integrable. Since in many important examples in analysis one has non irreducible representations, we allow for this situation to happen. 
Many relevant properties concerning reproducing systems are expressed efficiently with the notion of weak integral. We do not develop this theory in full here, but simply record what is needed to us. Suppose that $\Psi: G \rightarrow \mathcal{H}$ is a continuous map and suppose further that for any $v \in \mathcal{H}$ the integral

$$
\int_{G}\langle\Psi(x), v\rangle d x
$$

is absolutely convergent for every $v \in \mathcal{H}$. Then, as a consequence of the closed graph theorem, the mapping $v \mapsto \int_{G}\langle\Psi(x), v\rangle d x$ defines a continuous linear functional on $\mathcal{H}$. We collect these two properties by saying that $\Psi$ is scalarly continuously integrable. Then by the Riesz representation theorem there exists a unique element in $\mathcal{H}$, denoted

$$
\int_{G} \Psi(x) d x
$$

and called the weak integral of $\Psi$, for which

$$
\left\langle\int_{G} \Psi(x) d x, v\right\rangle=\int_{G}\langle\Psi(x), v\rangle d x, \quad v \in \mathcal{H} .
$$

Proposition 1.58. Suppose that $(G, \pi, \mathcal{H}, u)$ is a reproducing system. Then the reproducing formula

$$
v=\int_{G}\langle v, \pi(x) u\rangle \pi(x) u d x
$$

holds for $v \in \mathcal{H}$, where the right hand side is interpreted as weak integral. The adjoint of the voice transform is given as weak integral by the formula

$$
V_{u}^{*} F=\int_{G} F(x) \pi(x) u d x, \quad F \in L^{2}(G)
$$

and $V_{u}^{*} V_{u}=\mathrm{id}_{\mathcal{H}}$.

Proof. Since the voice transform $V_{u}$ maps $\mathcal{H}$ into $L^{2}(G)$ by assumption, and since it satisfies the isometric property (1.52), for every $w \in \mathcal{H}$ we have

$$
\begin{aligned}
\int_{G}\langle v, \pi(x) u\rangle\langle\pi(x) u, w\rangle d x & =\int_{G} V_{u} v(x) \overline{V_{u} w(x)} d x \\
& =\left\langle V_{u} v, V_{u} w\right\rangle \\
& =\langle v, w\rangle .
\end{aligned}
$$

This shows that the continuous mapping $\Psi_{v}: G \rightarrow \mathcal{H}$ defined for fixed $v \in \mathcal{H}$ by

$$
\Psi_{v}(x)=\langle v, \pi(x) u\rangle \pi(x) u
$$

is scalarly continuously integrable, because $w \mapsto\langle v, w\rangle$ is well defined and continuous. Hence $\Psi_{v}$ is weakly integrable, and the weak integral of $\Psi_{v}$ must be equal to $v$ because (1.56) entails

$$
\left\langle\int_{G} \Psi_{v}(x) d x, w\right\rangle=\langle v, w\rangle
$$


for every $w \in \mathcal{H}$. This establishes (1.54). As for (1.55), we notice that for any $F \in L^{2}(G)$ the continuous mapping $\Phi_{F}: G \rightarrow \mathcal{H}$ defined by $\Phi_{F}(x)=F(x) \pi(x) u$ is scalarly continuously integrable because

$$
\int_{G}\langle F(x) \pi(x) u, w\rangle d x=\int_{G} F(x) \overline{V_{u} w(x)} d x=\left\langle F, V_{u} w\right\rangle .
$$

Formula $V_{u}^{*} V_{u}=\operatorname{id}_{\mathcal{H}}$ follows from (1.55) applied to $F=V_{u} v$ and from (1.54), for

$$
V_{u}^{*} V_{u} v=\int_{G} V_{u} v(x) \pi(x) u d x=\int_{G}\langle v, \pi(x) u\rangle \pi(x) u d x=v .
$$

Before we proceed further, some comments are in order. The first observation concerns the geometric interpretation of (1.54). The mapping $\Psi_{v}$ defined in (1.57) associates to $x \in G$ the projection of $v$ along $\pi(x) u$. The reproducing formula (1.54) then expresses the fact that we can recover any element $v \in \mathcal{H}$ by gluing all its projections with an integral, so that in some sense the collection of all the vectors $\{\pi(x) u: x \in G\}$, called the orbit of $u$ under $G$, consists of sufficiently many "directions".

Secondly, the weak integral (1.55) that defines the adjoint of the voice transform is at times referred to as the Fourier transform of $F$ evaluated at $u$, and is written

$$
V_{u}^{*} F=\pi(F) u \text {. }
$$

A third comment concerns general properties of isometries. As already mentioned in the beginning of Section 1.3, a bounded linear operator $T: \mathcal{H}_{1} \rightarrow \mathcal{H}_{2}$ between Hilbert spaces is an isometry if and only if $T^{*} T=\mathrm{id}_{\mathcal{H}_{1}}$. Thus, the last statement in the previous theorem is in fact a simple consequence of the fact that $V_{u}$ is an isometry. Furthermore, if $T$ is an isometry, then $T T^{*}$ is the projection onto the range of $T$, for $T T^{*}$ is selfadjoint and idempotent.

Example 1.59. The following is an important example of reproducing system: the (irreducible) wavelet system that was discussed in Section 1.49. We consider the full affine group " $a x+b$ ", where $a$ is any non zero real number and $b \in \mathbb{R}$. The representation $\pi_{\text {full }}$ is defined in (1.35) and the representation space is $\mathcal{H}=L^{2}(\mathbb{R})$. If $f \in L^{2}(\mathbb{R})$ satisfies the Calderón equation (1.46), then (1.45) becomes

$$
\int_{G_{\text {full }}}\left|\left\langle g, \pi_{\text {full }}(a, b) f\right\rangle\right|^{2} \frac{d a d b}{a^{2}}=\|g\|_{2}^{2},
$$

which shows that the voice transform $V_{f}$, whose explicit form is given by

$$
V_{f} g(a, b)=\frac{1}{\sqrt{|a|}} \int_{\mathbb{R}} g(x) \overline{f\left(\frac{x-b}{a}\right)} d x
$$

is indeed an isometry of $L^{2}(\mathbb{R})$ into $L^{2}(G)$. Thus, the Calderón equation (1.46) selects the admissible vectors for the wavelet representation, namely the wavelets. The reproducing formula (1.54) is often written as:

$$
g(x)=\int_{\mathbb{R}} \int_{\mathbb{R}^{*}} V_{f} g(a, b) T_{b} D_{a} f(x) \frac{d a d b}{a^{2}} .
$$

The reader is referred to $[9,16,21]$ for further reading on this very wide topic. 
The next result describes this operator as a convolution with the kernel $K_{u}$.

Proposition 1.60. Suppose that $(G, \pi, \mathcal{H}, u)$ is a reproducing system. Then the projection onto the range of the voice transform is given by

$$
V_{u} V_{u}^{*} F=F * K_{u}, \quad F \in L^{2}(G) .
$$

In particular, $K_{u}$ is a convolution idempotent, that is

$$
K_{u}=K_{u} * K_{u} .
$$

Proof. First of all, notice that $K_{u}=V_{u} u \in L^{2}(G)$ and $\check{K}_{u}=\overline{K_{u}} \in L^{2}(G)$, so that, by (iii) of Theorem 1.38 (with $p=q=2$ ), the convolution $F * K_{u}$ is well defined for every $F \in L^{2}(G)$. Therefore, taking into account the various properties, we have

$$
\begin{aligned}
V_{u}\left(V_{u}^{*} F\right)(x) & =\left\langle V_{u}^{*} F, \pi(x) u\right\rangle \\
& =\left\langle F, V_{u}(\pi(x) u)\right\rangle \\
& =\left\langle F, \lambda(x) V_{u} u\right\rangle \\
& =\left\langle F, \lambda(x) K_{u}\right\rangle \\
& =\int_{G} F(y) \overline{K_{u}\left(x^{-1} y\right)} d y \\
& =\int_{G} F(y) K_{u}\left(y^{-1} x\right) d y \\
& =F * K_{u}(x) .
\end{aligned}
$$

The second statement is obvious, because $K_{u}$ is of course in the range of $V_{u}$ and hence coincides with its projection onto the range.

1.5. Unbounded operators. In this section $\mathcal{H}$ is a fixed Hilbert space. We say that $T$ is an operator on $\mathcal{H}$ if it is a linear map defined on a linear subspace $\mathcal{D}(T) \subset \mathcal{H}$, called its domain with image $\mathcal{R}(T) \subset \mathcal{H}$, another linear suspace called its range. It is not assumed that $T$ is bounded or continuous. Of course, if $T$ is continuous, then it has a continuous extension on the closure of $\mathcal{D}(T)$ and hence on $\mathcal{H}$. In other words, in this case $T$ is the restriction to $\mathcal{D}(T)$ of some $\widetilde{T} \in \mathcal{B}(\mathcal{H})$. The graph of $T$ in $\mathcal{H} \times \mathcal{H}$ will be denoted $\mathcal{G}(T)$. Observe that a linear map $S$ is an extension of $T$ if and only if $\mathcal{G}(T) \subset \mathcal{G}(S)$, so that in this case we may write $T \subset S$. An operator is called closed if such is its graph. The closed graph theorem asserts that $T \in \mathcal{B}(\mathcal{H})$ if and only if $\mathcal{D}(T)=\mathcal{H}$ and $T$ is a closed operator.

Next we define the adjoint of $T$, denoted $T^{*}$. Its domain $\mathcal{D}\left(T^{*}\right)$ consists of all the vectors $y \in \mathcal{H}$ for which the linear functional

$$
x \mapsto\langle T x, y\rangle
$$

is continuous on $\mathcal{D}(T)$. Thus, if $y \in \mathcal{D}\left(T^{*}\right)$, then the Hahn-Banach theorem allows us to extend the functional in (1.62) to a continuous linear functional on $\mathcal{H}$ and hence there exists an element, denoted $T^{*} y$ for which

$$
\langle T x, y\rangle=\left\langle x, T^{*} y\right\rangle, \quad x \in \mathcal{D}(T) .
$$


Clearly, $T^{*} y$ is uniquely determined by (1.63) if and only if $\mathcal{D}(T)$ is dense in $\mathcal{H}$. We shall then define $T^{*}$ only for the densely defined operators $T$.

Exercise 1.61. Show that if $T$ is a densely defined operator, then $T^{*}$ is an operator on $\mathcal{H}$. Prove further that if $T \in \mathcal{B}(\mathcal{H})$, then the definition of $T^{*}$ coincides with the usual one. In particular, $\mathcal{D}\left(T^{*}\right)=\mathcal{H}$ and $T^{*} \in \mathcal{B}(\mathcal{H})$.

Exercise 1.62. Let $R, S$ and $T$ be operators on $\mathcal{H}$. Prove the following relations:

$$
\begin{aligned}
& \mathcal{D}(S+T)=\mathcal{D}(S) \cap \mathcal{D}(T) ; \\
& \mathcal{D}(S T)=\{x \in \mathcal{D}(T): T x \in \mathcal{D}(S)\} \\
& (R+S)+T=R+(S+T) \\
& (R S) T=R(S T) \\
& (R+S) T=R T+S T ; \\
& T R+T S \subset T(R+S) .
\end{aligned}
$$

Exercise 1.63. Let $S, T$ and $S T$ be densely defined operators on $\mathcal{H}$. Prove that then $T^{*} S^{*} \subset(S T)^{*}$. Furthermore, if $S \in \mathcal{B}(\mathcal{H})$, then $T^{*} S^{*}=(S T)^{*}$.

Definition 1.64. An operator on $\mathcal{H}$ is said to be symmetric if for every $x \in \mathcal{D}(T)$ and $y \in \mathcal{D}(T)$

$$
\langle T x, y\rangle=\langle x, T y\rangle .
$$

The symmetric densely defined operators are those for which

$$
T \subset T^{*} .
$$

If $T=T^{*}$, then $T$ is called selfadjoint. Finally, we say that $T$ is skewadjoint if iT is selfadjoint.

Observe that a bounded operator is symmetric if and only if it is selfadjoint. In general this is not true. Furthermore, if $\mathcal{D}(T)$ is dense and $\langle T x, y\rangle=\langle x, S y\rangle$ for every $x \in \mathcal{D}(T)$ and every $y \in \mathcal{D}(S)$, then $S \subset T^{*}$.

Example 1.65. Let $\mathcal{H}=L^{2}([0,1])$ with the Lebesgue, measure, and put:

$$
\begin{aligned}
& \mathcal{D}\left(T_{1}\right)=\left\{f \in A . C .[0,1]: f^{\prime} \in L^{2}\right\} \\
& \mathcal{D}\left(T_{2}\right)=\mathcal{D}\left(T_{1}\right) \cap\{f: f(0)=f(1)\} \\
& \mathcal{D}\left(T_{3}\right)=\mathcal{D}\left(T_{1}\right) \cap\{f: f(0)=f(1)=0\},
\end{aligned}
$$

where A.C.[0,1] is the space of absolutely continuous functions on $[0,1]$. Define next

$$
T_{k} f=i f^{\prime}, \quad f \in \mathcal{D}\left(T_{k}\right), k=1,2,3 .
$$

It is not hard to show that

$$
T_{1}^{*}=T_{3}, \quad T_{2}^{*}=T_{2}, \quad T_{3}^{*}=T_{1} .
$$

Since clearly $T_{3} \subset T_{2} \subset T_{1}$, it follows that $T_{2}$ is a selfadjoint extension of $T_{3}$, which is symmetric but not selfadjoint, and that the extension $T_{1}$ of $T_{2}$ is not symmetric. 
We now discuss a phenomenon that is relevant in the representation theory of the Heisenberg group. Take again $\mathcal{H}=L^{2}([0,1])$ as above and consider $D f=f^{\prime}$ on $\mathcal{D}\left(T_{2}\right)$ and $M f(t)=t f(t)$. It is immediate to check that $(D M-M D) f=f$, that is

$$
D M-M D=I,
$$

where $I$ is the identity on the domain of $D$. Thus, the identity appears as the commutator of two operators, only one of which is bounded $\left(\|M f\|_{2} \leqslant\|f\|_{2}\right.$ because $\left.t \in[0,1]\right)$.

One can legitimately ask if it is possible to realize an equality like (1.66) with two bounded operators. The answer is negative, not only in the Banach algebra $\mathcal{B}(\mathcal{H})$ but in any other Banach algebra with unit. The very elegant proof of the proposition that follows is due to Wielandt.

Theorem 1.66. Let $A$ be a Banach algebra with unit $e$. If $x, y \in A$, then

$$
x y-y x \neq e .
$$

Proof. Suppose that $x y-y x=e$ and let us make the inductive assumption

$$
x^{n} y-y x^{n}=n x^{n-1},
$$

which is true for $n=1$. Then

$$
\begin{aligned}
x^{n+1} y-y x^{n+1} & =x^{n}(x y-y x)+\left(x^{n} y-y x^{n}\right) x \\
& =x^{n} e+n x^{n-1} x \\
& =(n+1) x^{n},
\end{aligned}
$$

so that the relation is true for all positive integers $n$. It then follows that

$$
n\left\|x^{n-1}\right\|=\left\|x^{n} y-y x^{n}\right\| \leqslant 2\left\|x^{n}\right\|\|y\| \leqslant 2\left\|x^{n-1}\right\|\|x\|\|y\|,
$$

that is $n \leqslant 2\|x\|\|y\|$ for every $n$. This is impossible.

1.6. Stone's theorem and the differential of a representation. In this section we state Stone's theorem, an infinite dimensional analogue of the fact that the Lie algebra of the unitary group is the skew-hermitian matrices. We then explain how to any unitary representation of a Lie group $G$ there corresponds a representation of its Lie algebra by "skew hermitian", i.e. skewadjoint, operators.

Definition 1.67. A one parameter group of operators on $\mathcal{H}$ is a family $\left\{U_{t}: t \in \mathbb{R}\right\} \subset$ $\mathcal{B}(\mathcal{H})$ that satisfies

i) $U_{0}=I$;

ii) $U_{t+s}=U_{t} U_{s}$;

iii) $\lim _{t \rightarrow 0}\left\|U_{t} x-x\right\|=0$ for every $x \in \mathcal{H}$

If $\pi$ is a unitary representation on $\mathcal{H}$ of the Lie group $G$ with Lie algebra $\mathfrak{g}$, then for any fixed $X \in \mathfrak{g}$ the family $\left\{U_{t}=\pi(\exp t X): t \in \mathbb{R}\right\}$ is a one parameter group of unitary operators on $\mathcal{H}$.

In analogy with the case $\mathcal{H}=\mathbb{C}$, in which every differentiable function such that $f(s+t)=f(s) f(t)$ is of the form $f(t)=e^{a t}$ with $a=f^{\prime}(0)$, it is possible to associate to any one parameter group of operators a "generating" operator, in general unbounded, as explained in the definition that follows. 
Definition 1.68. Let $\left\{U_{t}: t \in \mathbb{R}\right\}$ be a one parameter group of operators on $\mathcal{H}$ and let $\mathcal{D}(A)$ denote the subspace of $\mathcal{H}$ consisting of the vectors $x \in \mathcal{H}$ for which

$$
\lim _{t \rightarrow 0} \frac{U_{t} x-x}{t}=: A x
$$

exists in the norm topology of $\mathcal{H}$. The operator $A$ (necessarily linear) defined on $\mathcal{D}(A)$ by (1.67) is called the infinitesimal generator of the group.

Below is the classical statement of the celebrated theorem by M.H. Stone. For a proof see for instance [20].

Theorem 1.69. (Stone's theorem) Let $\left\{U_{t}\right\}$ be a one parameter group of unitary operators on $\mathcal{H}$. The infinitesimal generator $A$ of $\left\{U_{t}\right\}$ is densely defined on $\mathcal{H}$ and is skewadjoint. Conversely, if $A$ is a densely defined operator on $\mathcal{H}$ and is skewadjoint, then there exits a unique one parameter group of unitary operators on $\mathcal{H}$ whose infinitesimal generator is $A$.

Let's go back to the case when $\mathcal{H}$ is the space on which the unitary representation $\pi$ of the Lie group $G$ acts. By means of (1.67), we define the differential $d \pi$ on $\mathfrak{g}$ as

$$
d \pi(X) x=\lim _{t \rightarrow 0} \frac{\pi(\exp t X) x-x}{t}
$$

which we sometime write as

$$
d \pi(X) x=\left.\frac{d}{d t}\right|_{t=0} \pi(\exp t X) x .
$$

Our next objective is to show that $d \pi$ indeed defines a representation of $\mathfrak{g}$, that is, to make sure that the various operators $d \pi(X)$ can be properly composed and satisfy

$$
d \pi([X, Y])=d \pi(X) \circ d \pi(Y)-d \pi(Y) \circ d \pi(X) .
$$

We must therefore study the domains $\mathcal{D}(d \pi(X))$.

Recall that a function $f$ defined on an open $\Omega \in \mathbb{R}^{d}$ with values in $\mathcal{H}$ is differentiable at $x_{0} \in \Omega$ if there is a linear map $d f_{x_{0}}: \mathbb{R}^{d} \rightarrow \mathcal{H}$, necessarily unique, such that

$$
\lim _{x \rightarrow x_{0}} \frac{f(x)-f\left(x_{0}\right)-d f_{x_{0}}\left(x-x_{0}\right)}{\left\|x-x_{0}\right\|}=0,
$$

where $\|\cdot\|$ is any norm in $\mathbb{R}^{d}$. The map $d f_{x_{0}}$ is then the differential of $f$ at $x_{0}$. If $f$ is differentiable at all points of $\Omega$, then $x \mapsto d f_{x}$ is a map from $\Omega$ into $\operatorname{End}\left(\mathbb{R}^{d}, \mathcal{H}\right)$. The latter is, in turn, is a topological vector space in a canonical way. We then say that $f$ is of class $C^{1}$ if $x \mapsto d f_{x}$ is continuous, of class $C^{2}$ if $x \mapsto d f_{x}$ is of class $C^{1}$, and so on. We say that $f$ is of class $C^{\infty}$ if it is of class $C^{k}$ for all $k$.

The notion of $C^{\infty}$ map is clearly local and applies to the case of maps defined on a Lie group $G$ with values in $\mathcal{H}$. The following result establishes an analogue of formula (1.10) adapted to this setup. 
Proposition 1.70. Let $G$ be a Lie group with Lie algebra $\mathfrak{g}$ and take $X \in \mathfrak{g}$. If $F: G \rightarrow \mathcal{H}$ is a $C^{\infty}$ map with values in the Hilbert space $\mathcal{H}$, then the map

$$
g \mapsto X_{g} F=\lim _{t \rightarrow 0} \frac{F(g \exp t X)-F(g)}{t}
$$

is also of class $C^{\infty}$.

Proof. The composition $(g, t) \mapsto(g, \exp t X) \mapsto g \exp t X \mapsto F(g \exp t X)$ is $C^{\infty}$. Hence, its partial derivative with respect to $t$ at zero is $C^{\infty}$ with respect to $g$.

We can finally introduce the space on which the operators that arise from the differential of a unitary representation of a Lie group are naturally defined.

Definition 1.71. Let $\pi$ be a unitary representaiton of the Lie group $G$ on $\mathcal{H}$. An element $\xi \in \mathcal{H}$ is called a $C^{\infty}$-vector for $\pi$ if $g \mapsto \pi(g) \xi$ is of class $C^{\infty}$ on $G$. The space of $C^{\infty}$-vectors for $\pi$ will be denoted $C^{\infty}(\pi)$.

Theorem 1.72. Let $\pi$ be a unitary representation of the Lie group $G$ on the Hilbert space $\mathcal{H}$. For any $X \in \mathfrak{g}, d \pi(X)$ sends $C^{\infty}(\pi)$ into itself and

$$
d \pi([X, Y]) \xi=(d \pi(X) \circ d \pi(Y)-d \pi(Y) \circ d \pi(X)) \xi, \quad \xi \in C^{\infty}(\pi) .
$$

From Theorem 1.72 it is easy to derive the following facts.

Proposition 1.73. Let $\pi$ be a unitary representation of the Lie group $G$ on the Hilbert space $\mathcal{H}$ and let $\mathfrak{g}$ denote the Lia algebra of $G$. Then:

i) for every $X \in \mathfrak{g}$, the operator $d \pi(X)$ is skew symmetric on $C^{\infty}(\pi)$;

ii) for every $g \in G, \pi(g)$ sends $C^{\infty}(\pi)$ into itself;

iii) for every $g \in G$ and every $X \in \mathfrak{g}$ the formula $\pi(g) d \pi(X) \pi(g)^{-1}=d \pi(\operatorname{Ad} g X)$ holds.

We conclude this section with a crucial result, which implies that the operators $d \pi(X)$ are densely defined on $\mathcal{H}$. For a proof, see for instance [18]

Theorem 1.74. Let $\pi$ be a unitary representation of the Lie group $G$ on the Hilbert space $\mathcal{H}$. The space $C^{\infty}(\pi)$ is dense in $\mathcal{H}$. 


\section{The Heisenberg Group AND its RePRESENTAtions}

2.1. The group and its Lie algebra. We shall denote by $\mathbb{H}^{d}$ the Heisenberg group, namely the smooth manifold $\mathbb{R}^{d} \times \mathbb{R}^{d} \times \mathbb{R}$ endowed with the product

$$
\left((q, p, t)\left(q^{\prime}, p^{\prime}, t^{\prime}\right)=\left(q+q^{\prime}, p+p^{\prime}, t+t^{\prime}-\frac{1}{2}\left({ }^{t} q p^{\prime}-{ }^{t} p q^{\prime}\right)\right) .\right.
$$

Let $\omega: \mathbb{R}^{2 d} \times \mathbb{R}^{2 d} \rightarrow \mathbb{R}$ be the standard symplectic form given by (1.2), that is

$$
\omega\left(x, x^{\prime}\right)={ }^{t} x J x^{\prime}
$$

Upon writing $x=x_{(q, p)}={ }^{t}[q, p] \in \mathbb{R}^{2 d}$, we may formulate (2.72) in terms of the symplectic form, namely:

$$
\left((x, t)\left(x^{\prime}, t^{\prime}\right)=\left(x+x^{\prime}, t+t^{\prime}-\frac{1}{2} \omega\left(x, x^{\prime}\right)\right) .\right.
$$

It is clear from (2.72) and (2.74) that the product in $\mathbb{H}^{d}$ is given by functions that are $C^{\infty}$ in the global $\mathbb{R}^{2 d+1}$ coordinates $(q, p, t)$. Furthermore, one checks at once that

$$
(x, t)^{-1}=(-x,-t),
$$

another $C^{\infty}$ formula. Hence $\mathbb{H}^{d}$ is a Lie group.

Observe that $\mathbb{H}^{1}$ can also be seen as a particular group of symplectic matrices, that is, a subgroup of $S p(2, \mathbb{R})$, in the sense of (1.1). Indeed, if we put

$$
g(q, p, t)=\left[\begin{array}{cccc}
1 & 0 & 0 & 0 \\
p & 1 & 0 & 0 \\
t & -q / 2 & 1 & -p \\
-q / 2 & 0 & 0 & 1
\end{array}\right],
$$

then it is easy to check that the very same product formula as in (2.74) holds true.

Exercise 2.1. Prove that the center of $\mathbb{H}^{d}$ is $Z=\{(0, t): t \in \mathbb{R}\}$. Show that the quotient group $\mathbb{H}^{d} / Z$ is isomorphic to the Abelian Lie group $\mathbb{R}^{2 d}$.

Exercise 2.2. Write explicitely the inner conjugation $i_{g}(h)=g h g^{-1}$.

Exercise 2.3. Prove that $L_{1}=\left\{(q, 0, t): q \in \mathbb{R}^{d}, t \in \mathbb{R}\right\}$ and $L_{2}=\{(0, p, t)$ : $\left.p \in \mathbb{R}^{d}, t \in \mathbb{R}\right\}$ are two Lie subgroups of $\mathbb{H}^{d}$ which are mutually isomorphic but not conjugate.

Exercise 2.4. Check that the matrices in $(2.75)$ are in $S p(2, \mathbb{R})$ and that they satisfy the product law (2.74).

Exercise 2.5. Extend the embedding (2.75) to arbitrary dimension.

We next want to identify the Lie algebra $\mathfrak{h}^{d}$ of $\mathbb{H}^{d}$ in terms of left invariant vector fields. To this end, fix $(q, p, t) \in \mathbb{H}^{d}$ and $f \in C^{\infty}\left(\mathbb{H}^{d}\right)$ and consider the smooth curve $q_{j}(s)=\left(s e_{j}, 0,0\right)$, with $s \in(-\varepsilon, \varepsilon)$, where $e_{j}$ denotes the $j$-th unit coordinate vector in $\mathbb{R}^{d}$. Then

$$
\begin{aligned}
\left.\frac{d}{d s}\right|_{s=0} f\left((q, p, t)\left(s e_{j}, 0,0\right)\right. & =\left.\frac{d}{d s}\right|_{s=0} f\left(q+s e_{j}, p, t+\frac{1}{2} s p_{j}\right) \\
& =\frac{\partial f}{\partial q_{j}}(q, p, t)+\frac{1}{2} p_{j} \frac{\partial f}{\partial t}(q, p, t) .
\end{aligned}
$$


Hence, the equivalence class of the curve $q_{j}$ at $(q, p, t)$ is the differential operator

$$
\left.\frac{\partial}{\partial q_{j}}\right|_{(q, p, t)}+\left.\frac{1}{2} p_{j} \frac{\partial}{\partial t}\right|_{(q, p, t)}
$$

An analogous calculation with the curves $p_{j}(s)=\left(0, s e_{j}, 0\right)$ and $t(s)=(0,0, s)$ shows that a basis for $\mathfrak{h}^{d}$ is given by the vector fields $\left\{Q_{1}, \ldots, Q_{d}, P_{1}, \ldots, P_{d}, T\right\}$, where

$$
\begin{array}{rlr}
Q_{j} & =\frac{\partial}{\partial q_{j}}+\frac{1}{2} p_{j} \frac{\partial}{\partial t}, \quad j=1, \ldots, d \\
P_{j} & =\frac{\partial}{\partial p_{j}}-\frac{1}{2} q_{j} \frac{\partial}{\partial t}, \quad j=1, \ldots, d \\
T & =\frac{\partial}{\partial t} .
\end{array}
$$

It is also straightforward to check that

$$
\begin{gathered}
{\left[Q_{j}, P_{k}\right]=-\delta_{j k} T, \quad j, k=1, \ldots, d} \\
{\left[Q_{j}, T\right]=\left[P_{j}, T\right]=0, \quad j=1, \ldots, d}
\end{gathered}
$$

the celebrated Heisenberg commutation relations. Therefore, identifying $\mathbb{R}^{2 d+1}$ with $\mathfrak{h}^{d}$ via the map $\left(x_{1}, \ldots, x_{d}, y_{1}, \ldots, y_{d}, z\right) \mapsto \sum_{j=1}^{d}\left(x_{j} Q_{j}+y_{j} P_{j}\right)+z T$, we obtain the following Lie algebra structure on $\mathbb{R}^{2 d+1}$ :

$$
\left[(X, z),\left(X^{\prime}, z^{\prime}\right)\right]=\left(0,-\omega\left(X, X^{\prime}\right)\right) .
$$

From this commutator rule, one sees immediately that $[A,[B, C]]=0$ for every choice of $A, B$ and $C$ in $\mathfrak{h}^{d}$. Similarly, any higher order bracket vanishes. This fact is expressed technically by saying that $\mathfrak{h}^{d}$ is a two-step nilpotent Lie algebra. The BakerCampbell-Hausdorff formula (1.13) becomes $A, B \in \mathfrak{h}^{d}$

$$
\exp A \exp B=\exp \left(A+B+\frac{1}{2}[A, B]\right)
$$

and since for $A=(X, z)$ and $B=\left(X^{\prime}, z^{\prime}\right)$ it holds

$$
A+B+\frac{1}{2}[A, B]=\left(X+X^{\prime}, z+z^{\prime}-\frac{1}{2} \omega\left(X, X^{\prime}\right)\right),
$$

which coincides with the product (2.74), we infer that

$$
\exp (X, z)=(X, z) \quad \text { for every }(X, z) \in \mathbb{R}^{2 d+1} .
$$

This simply says that the exponential mapping is nothing else but the identity map of $\mathbb{R}^{2 d+1}$, when we identify the latter with $\mathfrak{h}^{d}$ on the one hand and with $\mathbb{H}^{d}$ on the other hand.

Exercise 2.6. Prove that the adjoint action of $\mathbb{H}^{d}$ on $\mathfrak{h}^{d}$ is given by

$$
\operatorname{Ad}(y, s)(X, t)=(X, t-\omega(y, X)) .
$$


Automorphisms. Given a Lie group $G$ with Lie algebra $\mathfrak{g}$ it is often interesting (and many times difficult) to understand their automorphism groups. Recall that an automorphism of the Lie group $G$ is an invertible group homomorphism. Unravelling the definition, this means a bijective smooth map $\varphi: G \rightarrow G$ preserving products, namely satisfying $\varphi(x y)=\varphi(x) \varphi(y)$ and $\varphi(e)=e$. The collection of all such maps is, in turn, a group under composition, denoted $\operatorname{Aut}(G)$. Under favorable circumstances ${ }^{2}$, and surely this is the case for $\mathbb{H}^{d}$, the group $\operatorname{Aut}(G)$ has itself a natural structure of Lie group, though we shall not insist on this. Notice that since we have identified $\mathbb{H}^{d}$ with $\mathbb{R}^{2 d+1}$, we are in fact looking at maps $\varphi: \mathbb{R}^{2 d+1} \rightarrow \mathbb{R}^{2 d+1}$.

Similarly, one may consider the automorphisms of $\mathfrak{g}$, namely the bijective linear maps $\Phi: \mathfrak{g} \rightarrow \mathfrak{g}$ satisfying $\Phi([X, Y])=[\Phi(X), \Phi(Y)]$. As we know, they form a group, denoted by $\operatorname{Aut}(\mathfrak{g})$. Since its elements are linear maps, $\operatorname{Aut}(\mathfrak{g})$ is in fact a (closed) subgroup of $G L(d, \mathbb{R})$ in a natural fashion, where $d$ is the dimension of $\mathfrak{g}$. Hence $\operatorname{Aut}(\mathfrak{g})$ can be given the structure of a Lie group without problems. Also, the differential $d \varphi$ of any $\varphi \in \operatorname{Aut}(G)$ is easily seen to belong to $\operatorname{Aut}(\mathfrak{g})$. This accounts for an immersion of $\operatorname{Aut}(G)$ into $\operatorname{Aut}(\mathfrak{g})$ whenever $G$ is connected. When looking at the Heisenberg group, things are even nicer than this, and the following results show exactly how. Very clear proofs of the statements that follow can be found in [15].

Proposition 2.7. Aut $\left(\mathbb{H}^{d}\right)=\operatorname{Aut}\left(\mathfrak{h}^{d}\right)$.

It is actually possible to give an explicit description of the automorphisms of $\mathbb{H}^{d}$. It is immediate to see that each of the following families of maps are automorphisms:

(i) Symplectic maps: For any $A \in S p(d, \mathbb{R}),\left(\left[\begin{array}{l}q \\ p\end{array}\right], t\right) \mapsto\left(A\left[\begin{array}{l}q \\ p\end{array}\right], t\right)$.

(ii) Inner automorphisms.

(iii) Homogeneous dilations: For any $a \in \mathbb{R}_{+}, \delta_{a}(x, t)=\left(a x, a^{2} t\right)$. Observe that for any $a, a^{\prime}>0$ it holds $\delta_{a} \delta_{a}=\delta_{a a^{\prime}}$.

(iv) Inversion: $(q, p, t) \mapsto(p, q,-t)$.

We shall momentarily denote by $G_{j}$ the automorphism group generated by the trasformations of type $j$, with $j \in\{(i),(i i),(i i i),(i v)\}$.

Theorem 2.8. Every automorphism of $\mathbb{H}^{d}$ can be written uniquely as $\alpha_{1} \alpha_{2} \alpha_{3} \alpha_{4}$, with $\alpha_{j} \in G_{j}$.

It is important to observe that the only automorphisms that leave the center fixed are those of the kind $(i)$ e $(i i)$. We shall denote by $\mathcal{T}$ the group generated by them.

Exercise 2.9. Define the semidirect product $\mathbb{R}^{2 d} \rtimes S p(d, \mathbb{R})$ as the set $\mathbb{R}^{2 d} \times S p(d, \mathbb{R})$ endowed with the product

$$
(x, A)\left(x^{\prime}, A^{\prime}\right)=\left(x+A x^{\prime}, A A^{\prime}\right) .
$$

\footnotetext{
${ }^{2}$ As it was shown by Hochschild in 1951, it is enough that the group of components of $G$ is finitely generated.
} 
Show that the map $(x, A) \mapsto \Psi_{(x, A)}$, where

$$
\Psi_{(x, A)}(y, t)=i_{(x, 0)}(A y, t), \quad(x, 0),(y, t) \in \mathbb{H}^{d}
$$

defines an isomorphism of $\mathbb{R}^{2 d} \rtimes S p(d, \mathbb{R})$ with $\mathcal{T}$. Define further the semidirect product $\mathbb{H}^{d} \rtimes S p(d, \mathbb{R})$ via the formula

$$
((x, t) ; A)\left(\left(x^{\prime}, t^{\prime}\right) ; A^{\prime}\right)=\left((x, t)\left(A x^{\prime}, t^{\prime}\right) ; A A^{\prime}\right) .
$$

Calculate its center $Z$ and prove that $\mathbb{H}^{d} \ltimes S p(d, \mathbb{R}) / Z$ is isomorphic to $\mathbb{R}^{2 d} \ltimes S p(d, \mathbb{R})$.

Exercise 2.10. Consider the symplectic matrix

$$
\varphi_{A}=\left[\begin{array}{cccc}
1 & 0 & 0 & 0 \\
0 & d & 0 & -2 c \\
0 & 0 & 1 & 0 \\
0 & -b / 2 & 0 & a
\end{array}\right] \in S p(2, \mathbb{R})
$$

where $a d-b c=1$, so that

$$
A=\left[\begin{array}{ll}
a & b \\
c & d
\end{array}\right] \in S L(2, \mathbb{R}) .
$$

Using the embedding (2.75), show that $\varphi_{A} g(q, p, t) \varphi_{A}^{-1}=g\left(q^{\prime}, p^{\prime}, t\right)$ where

$$
\left[\begin{array}{l}
q^{\prime} \\
p^{\prime}
\end{array}\right]=\left[\begin{array}{ll}
a & b \\
c & d
\end{array}\right]\left[\begin{array}{l}
q \\
p
\end{array}\right] .
$$

Thus the automorphisms of type (i) of $\mathbb{H}^{1}$ can be realized inside $S p(2, \mathbb{R})$ as conjugations. The six dimensional subgroup of $S p(2, \mathbb{R})$ generated by the matrices $g(q, p, t)$ and $\varphi_{A}$ is the so-called Jacobi group. Show that the Jacobi group is isomorphic to the semidirect product $\mathbb{H}^{1} \ltimes S L(2, \mathbb{R})$ of Exercise 2.9 for $d=1$, where the group law is given in (2.79). More on the Jacobi group is to be found in [3]. A classification of its subgroups up to conjugation is given in [12]. From the point of view of square integrability issues, it has been studied in [13].

Exercise 2.11. Show how to realize the automorphisms of type (ii) inside $S p(2, \mathbb{R}$ ) as conjugations.

Exercise 2.12. Consider next the symplectic matrix

$$
\Delta_{a}=\left[\begin{array}{cccc}
a^{-1} & 0 & 0 & 0 \\
0 & 1 & 0 & 0 \\
0 & 0 & a & 0 \\
0 & 0 & 0 & 1
\end{array}\right]
$$

Prove that $\Delta_{a} g \Delta_{a}^{-1}=\delta_{a}(g)$ for every $g$ as in (2.75). Thus, also the automorphisms of type (iii) of $\mathbb{H}^{1}$ can be realized inside $S p(2, \mathbb{R})$ as conjugations. The four dimensional subgroup of $S p(2, \mathbb{R})$ generated by the matrices $g(q, p, t)$ and $\Delta_{a}$ is isomorphic to the so-called extended Heisenberg group. The latter is the Heisenberg group extended by the group of homogeneous dilations, namely the semidirect product $\mathbb{H}^{d} \ltimes \mathbb{R}_{+}$where the product is

$$
(h, a)\left(h^{\prime}, a^{\prime}\right)=\left(h \delta_{a}\left(h^{\prime}\right), a a^{\prime}\right) .
$$

Exhibit the explicit isomorphism. 
2.2. The Schrödinger representation. Let $\pi$ be a unitary and irreducible representation of $\mathbb{H}^{d}$ on the Hilbert space $\mathcal{H}$. Then all the operators $\pi(0,0, t)$ are in $\mathcal{I}(\pi)$ because the center of $\mathbb{H}^{d}$ consists of the elements of the form $(0,0, t)$. Schur's Lemma implies that there exists a complex number of modulus one, denoted $\chi(t)$, such that

$$
\pi(0,0, t)=\chi(t) I_{\mathcal{H}}
$$

Therefore the function $t \mapsto \chi(t)$ is continuous from $\mathbb{R}$ into $\mathbb{T}$ and satisfies the equality $\chi(s+t)=\chi(s) \chi(t)$. Thus there exists a unique $\lambda \in \mathbb{R}$ such that

$$
\chi(t)=e^{i \lambda t} \text {. }
$$

Now, if $\lambda=0$, then $\pi(0,0, t)=I_{\mathcal{H}}$, so that $Z \subset \operatorname{ker} \pi$ and the representaion $\pi$ projects onto a representation $\tilde{\pi}$ on the quotient $\mathbb{H}^{d} / Z \simeq \mathbb{R}^{2 d}$. The representation $\tilde{\pi}$ is still unitary and irreducible. But $\mathbb{R}^{2 d}$ is Abelian and hence, by Corollary $1.55, \operatorname{dim} \mathcal{H}=1$, namely $\mathcal{H}=\mathbb{C}$. Therefore, there exists a unique vector $(\xi, \eta) \in \mathbb{R}^{2 d}$ such that

$$
\tilde{\pi}(x, y)=e^{i(\xi \cdot x+\eta \cdot y)} I_{\mathbb{C}}
$$

and hence $\pi$ acts on $\mathbb{C}$ as

$$
\pi(x, y, t) z=e^{i(\xi \cdot x+\eta \cdot y)} z .
$$

Consider next the case $\lambda \neq 0$. From (2.80) e (2.81), taking the differential, it follows

$$
d \pi(T)=i \lambda I_{\mathcal{H}}
$$

In particular $d \pi(T)$ is a bounded operator. But we also know that for every $j=1, \ldots, d$ we have $\left[Q_{j}, P_{j}\right]=-T$, so that necessarily

$$
\left[d \pi\left(Q_{j}\right), d \pi\left(P_{j}\right)\right]=-d \pi(T)=-i \lambda I_{\mathcal{H}} .
$$

By modifying Theorem 1.66 in the case of a multiple of the identity (the details are left as an exercise) we see that the operators $d \pi\left(Q_{j}\right)$ and $d \pi\left(P_{j}\right)$ cannot be both bounded and, in particular, the representation space $\mathcal{H}$ cannot be finite dimensional. In conclusion, every unitary and irreducible representation of $\mathbb{H}^{d}$ which is not trivial on the center is necessarily infinite dimensional.

The next step consists in looking for the unitary and irreducible representations of $\mathbb{H}^{d}$ that are not trivial on the center. The most natural way to go about it is to compare the commutation Heisenberg relations with (1.66). We shall start from a very natural representation of the Lie algebra $\mathfrak{h}^{d}$ and then use some heuristics, together with Stone's Theorem, in order to get the corresponding representation of the group. To this end, we introduce a useful Lie subalgebra of the Lie algebra of all differential operators with polynomial coefficients on $\mathbb{R}^{d}$.

Let $\mathcal{P} \mathcal{D}\left(\mathbb{R}^{d}\right)$ denote the vector space of all differential operators with polynomial coefficients on $\mathbb{R}^{d}$. Under composition, it is an associative algebra generated by the $2 d$ elements

$$
D_{j}=-\frac{\partial}{\partial x_{j}}, \quad M_{j}=(2 \pi i) x_{j}, \quad j=1, \ldots, d .
$$

As any other associative algebra, $\mathcal{P} \mathcal{D}\left(\mathbb{R}^{d}\right)$ becomes a Lie algebra if we define the bracket as the commutator, that is $[A, B]=A \circ B-B \circ A$. The generators $D_{j}$ and $M_{j}$, 
together with the operator " $2 \pi i$-times the identity", give rise to a finite dimensional Lie subalgebra of $\mathcal{P} \mathcal{D}\left(\mathbb{R}^{d}\right)$ which is isomorphic to $\mathfrak{h}^{d}$. Indeed, the correspondence $D_{j} \mapsto Q_{j}, M_{j} \mapsto P_{j}$ and $(2 \pi i) I \mapsto T$ establishes the isomorphism. This isomorphism is actually a faithful (i.e. injective) representation of the Heisenberg algebra and should be thought of as the differential of the representation we are looking for. In order to find the representation space, it will then be enough to find a Hilbert space of functions on $\mathbb{R}^{d}$ on which the operators we are dealing with are skew-adjoint. Finally, we shall exponentiate the representation of $\mathfrak{h}^{d}$.

Let $\mathcal{H}=L^{2}\left(\mathbb{R}^{d}\right)$ and $\mathcal{D}=\mathcal{S}\left(\mathbb{R}^{d}\right)$, the Schwartz space of rapidly decreasing functions. Both the derivations $D_{j}$ and the multiplications $M_{j}$ are densely defined on $\mathcal{H}$ since $\mathcal{D}$ is a natural common domain for them, which is dense in $\mathcal{H}$. The operators are formally skew-adjoint because for every $\varphi, \psi \in \mathcal{S}\left(\mathbb{R}^{d}\right)$ we have

$$
\left\langle M_{j} \varphi, \psi\right\rangle=-\left\langle\varphi, M_{j} \psi\right\rangle,\left\langle D_{j} \varphi, \psi\right\rangle=-\left\langle\varphi, D_{j} \psi\right\rangle,
$$

where we are using $L^{2}$ inner products and, in the second, integration by parts.

Observe that it is not necessary to specify the skew-adjoint extensions of $M_{j}$ and of $D_{j}$. It will be sufficient to exhibit one-parameter groups of unitary operators on $L^{2}\left(\mathbb{R}^{d}\right)$ whose infinitesimal generators extend our operators, for Stone's Theorem guarantees that these groups are the appropriate ones. Consider then the following formal computation:

$$
\begin{aligned}
\exp \left(t D_{j}\right) f(x) & =\sum_{n=0}^{\infty} \frac{\left(t D_{j}\right)^{n}}{n !} f(x) \\
& =\sum_{n=0}^{\infty} \frac{(-t)^{n}}{n !} \frac{\partial^{n} f}{\partial x_{j}^{n}}(x) \\
& =f\left(x-t e_{j}\right) .
\end{aligned}
$$

Similarly,

$$
\exp \left(t M_{j}\right) f(x)=e^{2 \pi i x_{j}} f(x) .
$$

It is also quite clear that the operators

$$
U_{t}^{(j)} f(x)=f\left(x-t e_{j}\right), \quad V_{t}^{(j)} f(x)=e^{2 \pi i t x_{j}} f(x)
$$

give rise to one-parameter groups of unitary operators on $L^{2}\left(\mathbb{R}^{d}\right)$.

Exercise 2.13. Prove that the Schwartz space $\mathcal{S}\left(\mathbb{R}^{d}\right)$ is contained in the domain of the infinitesimal generators of $\left\{U_{t}^{(j)}\right\}$ and $\left\{V_{t}^{(j)}\right\}$.

From the previous computations, it is natural to set:

$$
\begin{aligned}
& \pi(q, 0,0) f(x)=f(x-q) \\
& \pi(0, p, 0) f(x)=e^{2 \pi i p \cdot x} f(x) \\
& \pi(0,0, t) f(x)=e^{2 \pi i t} f(x) .
\end{aligned}
$$


Finally, since for every $(q, p, t) \in \mathbb{H}^{d}$ we have

$$
(q, p, t)=\left(0,0, t-\frac{1}{2} q \cdot p\right)(0, p, 0)(q, 0,0),
$$

by composing the various (2.84) we obtain

$$
\pi(q, p, t) f(x)=e^{2 \pi i t} e^{-\pi i q \cdot p} e^{2 \pi i p \cdot x} f(x-q) .
$$

It is now elementary to check that (2.85) defines a representation of $\mathbb{H}^{d}$, called the Schrödinger representation. While it is clear that the thus defined operators are unitary because they are compositions of unitary operators, irreducibility requires some extra work. Reall that for us the Fourier transform is as in (1.36).

Theorem 2.14. Formula (2.85) defines a unitary irreducible representation of $\mathbb{H}^{d}$ on $L^{2}\left(\mathbb{R}^{d}\right)$.

Proof. In order to show that $\pi$ is continuous in the strong operator topology it is enough to prove that if $(q, p, t) \rightarrow(0,0,0)$, then $\pi(q, p, t) f \rightarrow f$ in $L^{2}\left(\mathbb{R}^{d}\right)$ for every $f \in L^{2}\left(\mathbb{R}^{d}\right)$. Now,

$$
\begin{aligned}
\left(\int_{\mathbb{R}^{d}}|\pi(q, p, t) f(x)-f(x)|^{2} d x\right)^{1 / 2}= & \left(\int_{\mathbb{R}^{d}}\left|e^{2 \pi i t-\pi i q \cdot p+2 \pi i p \cdot x} f(x-q)-f(x)\right|^{2} d x\right)^{\frac{1}{2}} \\
\leqslant & \left(\int_{\mathbb{R}^{d}}\left|e^{2 \pi i t-\pi i q \cdot p+2 \pi i p \cdot x}-1\right|^{2}|f(x)|^{2} d x\right)^{\frac{1}{2}} \\
& +\left(\int_{\mathbb{R}^{d}}|f(x-q)-f(x)|^{2} d x\right)^{\frac{1}{2}}
\end{aligned}
$$

and both summands tend to zero as $(q, p, t) \rightarrow(0,0,0)$.

Let's show irreducibility. Take a closed subspace $\mathcal{M} \subseteq L^{2}\left(\mathbb{R}^{d}\right)$ and suppose that it is $\pi$-invariant. If $f \in \mathcal{M}$, then the translate $\tau_{q} f=\pi(q, 0,0) f$ in also in $\mathcal{M}$. If $P$ is the orthogonal projection onto $\mathcal{M}$, then $P$ commutes with translations. Hence there exists a multiplier $m \in L^{\infty}\left(\mathbb{R}^{d}\right)$ such that

$$
\mathcal{F}(P f)(\xi)=m(\xi) \mathcal{F} f(\xi) .
$$

Since $P^{2}=P$, then also $m^{2}=m$. Upon considering $\pi(0, p, 0)$, one sees that $P$ commutes also with multiplication by any of the characters $e_{p}(x)=e^{2 \pi i p \cdot x}$. Therefore, from (2.86) we infer

$$
\begin{aligned}
\mathcal{F}\left[P\left(e_{p} f\right)\right](\xi) & =m(\xi) \mathcal{F}\left[e_{p} f\right](\xi)=m(\xi) \mathcal{F} f(\xi-p), \\
\mathcal{F}\left[e_{p} P f\right](\xi) & =\mathcal{F}[P f](\xi-p)=m(\xi-p) \mathcal{F} f(\xi-p),
\end{aligned}
$$

which yield

$$
m(\xi)=m(\xi-p), \quad \text { for every } p \in \mathbb{R}^{d} .
$$

Hence $m$ is constant and since $m^{2}=m$ it must be either $m=0$, that is $\mathcal{M}=\{0\}$, or else $m=1$, that is $\mathcal{M}=L^{2}\left(\mathbb{R}^{d}\right)$.

By means of formulae (2.85), we have build a unitary and irreducible representation $\pi$ of $\mathbb{H}^{d}$ for which $\pi(0,0, t)=e^{2 \pi i t} I_{\mathcal{H}}$, that is, recalling (2.80) and (2.81), the representation corresponding to the case $\lambda=2 \pi$. It is now easy to define a representation 
corresponding to the real number $\lambda=2 \pi h$, with $h \in \mathbb{R} \backslash\{0\}$. It is enough to put

$$
\pi_{h}(q, p, t) f(x)=\pi(h q, p, h t) f(x)=e^{2 \pi i h t} e^{-\pi i q \cdot h p} e^{2 \pi i p \cdot x} f(x-h q) .
$$

The non zero real number $h$ that labels the Schrödinger representation $\pi_{h}$ is known as Planck's constant. For comments concerning the physical meaning of $h$ see [15].

Exercise 2.15. Check that $\pi_{h}$ is a unitary and irreducible representation of $\mathbb{H}^{d}$ for every $h \neq 0$, and that if $h \neq h^{\prime}$, then the corresponding representations are inequivalent.

The following celebrated theorem by M. Stone and J. von Neumann is of fundamental importance in harmonic analysis: it states that the representations that we have built so far exhaust, up to equivalence, the class of unitary and irreducible representation of $\mathbb{H}^{d}$. For a proof, see, for example, [15].

Theorem 2.16. (Stone-von Neumann) Every unitary and irreducible representation of $\mathbb{H}^{d}$ is unitarily equivalent to either one of the one-dimensional representations (2.82), or to a Schrödinger representation $\pi_{h}$ defined in (2.87).

It is worth observing that the determination of the Planck's constant is very simple: just compute the representation on the central elements $(0,0, t)$ : by $(2.87)$ this must be just $e^{2 \pi h t} I$.

2.3. Time-frequency analysis. In this section we indicate the basic role that the representation theory of the Heisenberg group plays in time-frequency analysis, in particular in the study of the so-called short-time Fourier transform (often abbreviated in STFT). The basic reference on this topic is the book [16].

We first introduce some basic ingredients of time-frequency analysis and then show the connections to the representation theory of the (reduced) Heisenberg group. The basic issue is that the STFT can be viewed in the framework of reproducing systems, as discussed in Section 1.4 In order to stress the link between the STFT and the representation theory of the Heisenberg group, we follow the convention of denoting by $x$ or $q$ the spatial variable of functions (or time for $d=1$ ) and by $\xi$ or $p$ the frequency variable. Also, we indicate with a dot the scalar product in $\mathbb{R}^{d}$.

2.3.1. Short time Fourier transform. The most basic operations in time-frequency analysis are the shifts in time and in frequency. For $q, p \in \mathbb{R}^{d}$ and $f: \mathbb{R}^{d} \rightarrow \mathbb{C}$, we define the time shift by

$$
T_{q} f(x)=f(x-q), \quad q, x \in \mathbb{R}^{d}
$$

and the frequency shift by

$$
M_{p} f(\xi)=e^{2 \pi i p \cdot \xi} f(\xi), \quad \xi, p \in \mathbb{R}^{d} .
$$

It is a matter of simple computation to establish a version of the canonical commutaion relations, namely

$$
T_{q} M_{p}=e^{-2 \pi i q \cdot p} M_{p} T_{q} .
$$


It follows in particular that $T_{q}$ and $M_{p}$ commute if and only if $q \cdot p \in \mathbb{Z}$. Furthermore, writing $\hat{f}$ for the Fourier transform $\mathcal{F} f$ defined in (1.36), and using the well-known properties of $\mathcal{F} f$, we have the formulae

$$
\left(T_{q} f\right)^{\wedge}=M_{-q} \hat{f}, \quad\left(M_{p} f\right)^{\wedge}=T_{p} \hat{f} .
$$

From these it follows what is to be regarded as the most important formula in timefrequency analysis, namely

$$
\left(T_{q} M_{p} f\right)^{\wedge}=M_{-q} T_{p} \hat{f}=e^{-2 \pi i q \cdot p} T_{p} M_{-q} \hat{f},
$$

whose proof is is left as an exercise.

The short-time Fourier transform is a mathematical device that is meant to capture the local contributions to the Fourier transform of a given function: one restricts the function to a small intervall by a cut-off function (preferably some smooth window) and then takes the Fourier transform; by sliding the interval, one sees what are the various contributions from different regions of the time domain. Formally, we have:

Definition 2.17. Let $\eta \neq 0$ be a fixed window, that is, a function defined on $\mathbb{R}^{d}$. The short-time Fourier transform of the function $f: \mathbb{R}^{d} \rightarrow \mathbb{C}$ with respect to $\eta$ is defined by

$$
S_{\eta} f(q, p)=\int_{\mathbb{R}^{d}} f(x) \overline{\eta(x-q)} e^{-2 \pi i p \cdot y} d x, \quad q, p \in \mathbb{R}^{d}
$$

whenever the integral makes sense.

A most fundamental observation is that the STFT is a function on the so-called phase-space, namely the $2 d$-space $\mathbb{R}^{2 d}$ in which times and frequencies simultaneously lie. In other words, it is a function of time and frequency. The time variable labels the "center" of the window and the frequency labels the point at which the Fourier transform is evaluated.

At this stage we do not insist much on the precise domains for $\eta$ or for $f$. The most basic properties of the STFT are given in the result that follows. The proof is easy.

Proposition 2.18. If $f, \eta \in L^{2}\left(\mathbb{R}^{d}\right)$, then $S_{\eta} f$ is uniformly continuous in $\mathbb{R}^{2 d}$ and

$$
\begin{aligned}
S_{\eta} f(q, p) & =\mathcal{F}\left(f \cdot T_{q} \bar{\eta}\right)(p) \\
& =\left\langle f, M_{p} T_{q} \eta\right\rangle \\
& =\left\langle\hat{f}, T_{p} M_{-q} \hat{\eta}\right\rangle \\
& =e^{-2 \pi i q \cdot p} S_{\hat{\eta}} \hat{f}(p,-q) .
\end{aligned}
$$

Some comments. Formula (2.94) says what the STFT really is, the Fourier transform of a localized version of $f$. Formulae (2.95) and (2.96) indicate that it looks formally like the coefficient of some representation. Formula (2.97) exhibits a most intriguing symmetry in time and frequency, together with a ninety-degree rotation $(q, p) \mapsto(p,-q)$ in phase-space (given by the action of the matrix $J$ ).

In the next three results we state some of the most remarkable features of the STFT. 
Theorem 2.19 (Orthogonality relations for the STFT). Let $f_{1}, f_{2}, \eta_{1}, \eta_{2} \in L^{2}\left(\mathbb{R}^{d}\right)$. Then $S_{\eta_{j}} f_{j} \in L^{2}\left(\mathbb{R}^{2 d}\right)$ for $j=1,2$ and

$$
\left\langle S_{\eta_{1}} f_{1}, S_{\eta_{2}} f_{2}\right\rangle=\left\langle f_{1}, f_{2}\right\rangle \overline{\left\langle\eta_{1}, \eta_{2}\right\rangle}
$$

It must be observed that the first inner product in $(2.98)$ is in $L^{2}\left(\mathbb{R}^{2 d}\right)$ (phase space) whereas the ones appearing in the right-hand side are both in $L^{2}\left(\mathbb{R}^{d}\right)$, in the time domain or in the frequency domain, as one prefers, because of Parseval's equality.

Corollary 2.20. If $f, \eta \in L^{2}\left(\mathbb{R}^{d}\right)$, then

$$
\left\|S_{\eta} f\right\|_{2}=\|f\|_{2}\|\eta\|_{2}
$$

In particular, if $\|\eta\|_{2}=1$, then

$$
\left\|S_{\eta} f\right\|_{2}=\|f\|_{2} \quad \text { for all } f \in L^{2}\left(\mathbb{R}^{d}\right)
$$

and in this case the STFT is an isometry of $L^{2}\left(\mathbb{R}^{d}\right)$ into $L^{2}\left(\mathbb{R}^{d}\right)$.

Theorem 2.21 (Inversion of the STFT). Let $\eta, \gamma \in L^{2}\left(\mathbb{R}^{d}\right)$ be such that $\langle\eta, \gamma\rangle \neq 0$. Then, for every $f \in L^{2}\left(\mathbb{R}^{d}\right)$

$$
f=\frac{1}{\langle\gamma, \eta\rangle} \iint_{\mathbb{R}^{2 d}} S_{\eta} f(q, p) M_{p} T_{q} \gamma d q d p
$$

holds as a weak integral.

It must be pointed out that in many cases one choses $\eta=\gamma$ and assumes further that $\|\eta\|_{2}=1$, so that $(2.100)$ simplifies to

$$
f=\iint_{\mathbb{R}^{2 d}} S_{\eta} f(q, p) M_{p} T_{q} \eta d q d p,
$$

a formula that is sometimes referred to as the reproducing formula for the STFT.

2.3.2. Square integrability and the reduced Heisenberg group. First of all,we show that

$$
\pi(q, p, t)=e^{2 \pi i t} e^{\pi i q \cdot p} T_{q} M_{p}
$$

Indeed, for any $f \in L^{2}\left(\mathbb{R}^{d}\right)$ we have, by (2.90) and (2.85)

$$
\begin{aligned}
e^{2 \pi i t} e^{\pi i q \cdot p} T_{q} M_{p} f(y) & =e^{2 \pi i t} e^{\pi i q \cdot p} e^{-2 \pi i q \cdot p} M_{p} T_{q} f(y) \\
& =e^{2 \pi i t} e^{-\pi i q \cdot p} e^{2 \pi i p \cdot y} T_{q} f(y) \\
& =e^{2 \pi i t} e^{-\pi i q \cdot p} e^{2 \pi i p \cdot y} f(y-q) \\
& =\pi(q, p, t) f(y) .
\end{aligned}
$$

Secondly, for fixed $\eta, f \in L^{2}\left(\mathbb{R}^{d}\right)$, the coefficient $\langle f, \pi(q, p, t) \eta\rangle$ of the Schrödinger representation satisfies, according to (2.101), (2.90) and (2.95)

$$
\langle f, \pi(q, p, t) \eta\rangle=e^{-2 \pi i t} e^{-\pi i q \cdot p}\left\langle f, T_{q} M_{p} \eta\right\rangle=e^{-2 \pi i t} e^{\pi i q \cdot p} S_{\eta} f(q, p),
$$

Therefore the STFT is nothing else but a multiple (with a complex number of modulus one) of the voice transform associated to the Schrödinger representation of $\mathbb{H}^{d}$. 
One extra step needs to be made to recast the main properties of the STFT in the language of reproducing systems. Indeed, the STFT of a funcion is not a function on the Heisenberg group but on $\mathbb{R}^{2 d}$, and, secondly, even if we include the phase factor $e^{-2 \pi i t}$ in the definition so that it does become a function on $\mathbb{H}^{d}$, then there would be a serious integrability issue because the right hand side of $(2.102)$ is not in $L^{2}\left(\mathbb{H}^{d}\right)$ since the integral of its square modulus would certainly diverge in the $t$ variable. Things can be fixed by introducing the reduced Heisenberg group.

We start by computing the kernel of the Schrödinger representation. Using formula (2.101), it is immediate that $\pi(q, p, t) f=f$ for every $f \in L^{2}\left(\mathbb{R}^{d}\right)$ if and only if $q=p=0$ and $t \in \mathbb{Z}$. Thus

$$
\operatorname{ker} \pi=\{(0,0, k): k \in \mathbb{Z}\} \simeq \mathbb{Z} .
$$

The reduced Heisenberg group is nothing else but $\mathbb{H}^{d} / \operatorname{ker} \pi$. To have a workable model, one simply puts $\mathbb{H}_{r}^{d}=\mathbb{R}^{2 d} \times \mathbb{T}$, where $\mathbb{T}$ are the complex numbers of modulus one, and defines

$$
(x, \theta)\left(x^{\prime}, \theta\right)=\left(x+x, \theta \theta^{\prime} e^{-\pi i \omega(x, x)}\right) .
$$

It is quite clear that the map

$$
(x, t) \mapsto\left(x, e^{2 \pi i t}\right)
$$

is a surjective homomorphism of $\mathbb{H}^{d}$ onto $\mathbb{H}_{r}^{d}$ whose kernel is exactly $\operatorname{ker} \pi$, so that we have established a model for the quotient $\mathbb{H}^{d} / \operatorname{ker} \pi$.

Exercise 2.22. Show that if we parametrize the elements of $\mathbb{H}_{r}^{d}$ by $\left(x, e^{2 \pi i \tau}\right)$ where $\tau \in[0,1)$, then a left Haar measure on $\mathbb{H}_{r}^{d}$ is $d x d \tau$.

By the very construction of $\mathbb{H}_{r}^{d}$, the Schrödinger representation projects onto an irreducible representation $\rho:=\tilde{\pi}$ of $\mathbb{H}_{r}^{d}$ on $L^{2}\left(\mathbb{R}^{d}\right)$. With slight abuse, it is called the Schrödinger representation $\rho$ of $\mathbb{H}_{r}^{d}$ and its explicit formula is

$$
\rho(q, p, \theta)=\theta e^{\pi i q \cdot p} T_{q} M_{p}, \quad(q, p, \theta) \in \mathbb{H}_{r}^{d} .
$$

Exercise 2.23. Derive a theorem that describes all the irreducible representations of $\mathbb{H}_{r}^{d}$ starting from the Stone-von Neumann theorem.

Finally, we compute the voice transform $V_{\eta}$ of $\rho$ and find from (2.102) that

$$
V_{\eta} f(q, p, \theta)=\langle f, \rho(q, p, \theta) \eta\rangle=\bar{\theta} e^{\pi i q \cdot p} S_{\eta} f(q, p)
$$

Therefore, appealing to the orthogonality relations in the form (2.99), we get

$$
\left\|V_{\eta} f\right\|_{L^{2}\left(\mathbb{H}_{r}^{d}\right)}=\int_{0}^{1} \int_{\mathbb{R}^{2 d}}\left|e^{-2 \pi i \tau} e^{\pi i q \cdot p} S_{\eta} f(x)\right|^{2} d q d p d \tau=\left\|S_{\eta} f\right\|_{2}=\|f\|_{2}\|\eta\|_{2} .
$$

This proves that any unit vector $\eta \in L^{2}\left(\mathbb{R}^{d}\right)$ is an admissible vector for the Schrödinger representation $\rho$ of $\mathbb{H}_{r}^{d}$.

Exercise 2.24. Write the inversion formula of the STFT as the reproducing formula (1.54) associated to the square integrable representation $\rho$ of $\mathbb{H}_{r}^{d}$. 


\section{The MEtaplectC REPRESENTATION}

The meteplectic representation is a double-valued unitary representation of the symplectic group $S p(d, \mathbb{R})$ on $L^{2}\left(\mathbb{R}^{d}\right)$, or, more technically speaking, a unitary representation of the double cover of $S p(d, \mathbb{R})$, otherwise known as the metaplectic group $\operatorname{Mp}(d)$. It has many other names in the literature, such as the oscillator representation, or the Segal-Shale-Weil representation, and it appears pervasively in mathematics. We point out right from the start that it is not irreducible: both the spaces $L_{e}^{2}\left(\mathbb{R}^{d}\right)$ and $L_{o}^{2}\left(\mathbb{R}^{d}\right)$ of even and odd (square integrable) functions, respectively, are closed and invariant, and on each of them the metaplectic representation is irreducible.

In very practical terms, the metaplectic representation assigns to each symplectic matrix a unitary operator on $L^{2}\left(\mathbb{R}^{d}\right)$, which is well-defined only up to a sign. This ambiguity, though mathematically important and not removable, plays a very mild role, if none at all, in many of the most important aspects in which it appears in Applied Harmonic Analysis. In particular, this ambiguity is irrelevant in the context of reproducing formulae, that is, when square integrability issues arise.

As hinted in the previous paragraph, the very definition of the metaplectic representation is troublesome, and there are different ways of going about it. For a thorough presentation of this topic, the reader is referred to $[15,16,19]$. Here we content ourselves with a discussion of the main features rather than delving into the full machinery of proofs. The reason is because our interest resides in the restriction of the metaplectic representation to a particular class of triangular Lie subgroups of $S p(d, \mathbb{R})$. This class, known as the class $\mathcal{E}$, contains a rich subclass of reproducing groups, with interesting new examples, as well as well known ones. This general theme of reproducing groups for the metaplectic representation started with $[12,13]$ and has then been investigated in a series of more recente papers $[1,2,5,6,7,8,10]$.

We start by summarizing some useful properties of the symplectic group and of its Lie algebra. A direct proof of most of the statements may be found in [15]. The most advanced ones, such as for example the Iwasawa decomposition, can be found for example in [17].

3.1. More on the symplectic group. As we have seen, the symplectic group is the group of invertible $2 d \times 2 d$ matrices preserving the standard symplectic form, that is, the skew symmetric form $\omega: \mathbb{R}^{2 d} \times \mathbb{R}^{2 d} \rightarrow \mathbb{R}$ given by the matrix $J$ defined in (1.2).

Exercise 3.1. Show that an invertible matrix $g$ satisfies $\omega(g x, g y)=\omega(x, y)$ for every $x, y \in \mathbb{R}^{2 d}$ if and only if ${ }^{t} g J g=J$.

Recall that, under the usual identifications, the Lie algebra $\mathfrak{s p}(d, \mathbb{R})$ of $S p(d, \mathbb{R})$ is explicitly described in (1.22) and (1.23). It is worth observing that from these it follows immediately that $X \in \mathfrak{s p}(d, \mathbb{R})$ if and only if ${ }^{t} X \in \mathfrak{s p}(d, \mathbb{R})$. In particular, we obtain that both $J X$ and $X J$ are symmetric. Indeed, ${ }^{t}(J X)={ }^{t} X^{t} J=-{ }^{t} X J=J X$ by ${ }^{t} X J+J X=0$. Similarly, one uses ${ }^{t} X \in \mathfrak{s p}(d, \mathbb{R})$ to show that $X J$ is symmetric.

The symplectic group has many interesting subgroups. Among many others, the compact group

$$
K=S p(d, \mathbb{R}) \cap O(2 d)
$$


which was implicitly introduced in the beginning of Section 1.2.3, where we also describe an explicit isomorphism of $K$ with the unitary group $U(d)$. We formalize this:

Proposition 3.2. $K=S p(d, \mathbb{R}) \cap O(2 d)$ is a maximal compact subgroup of $S p(d, \mathbb{R})$ and it is isomorphic to the unitary group $U(d)$ under the natural map induced on linear maps by the identification $(x, y) \mapsto x+i y$ of $\mathbb{R}^{2 d}$ with $\mathbb{C}^{d}$.

Other very important groups of $S p(d, \mathbb{R})$ are

$$
\begin{aligned}
& D=\left\{\left[\begin{array}{cc}
h & 0 \\
0 & { }^{t} h^{-1}
\end{array}\right]: \operatorname{det} h \neq 0\right\} \\
& S=\left\{\left[\begin{array}{ll}
I & 0 \\
\sigma & I
\end{array}\right]: \sigma \in \operatorname{Sym}(d, \mathbb{R})\right\} \\
& A=\left\{\left[\begin{array}{cc}
E & 0 \\
0 & E^{-1}
\end{array}\right]: E=\operatorname{diag}\left(e^{\lambda_{1}}, \ldots, e^{\lambda_{d}}\right), \lambda_{j} \in \mathbb{R}\right\}
\end{aligned}
$$

where evidently $\operatorname{Sym}(d, \mathbb{R})$ stands for the vector space of all symmetric $d \times d$ real matrices. Clearly, $D \simeq G L(d, \mathbb{R})$. Using $D$, we can embed any (closed) subgroup of $G L(d, \mathbb{R})$ into $S p(d, \mathbb{R})$ in a canonical fashion, like for example the special linear group $S L(d, \mathbb{R})$ or the special orthogonal group $S O(d)$

$$
\begin{gathered}
S L(d, \mathbb{R}) \hookrightarrow\left\{\left[\begin{array}{cc}
h & 0 \\
0 & { }^{t} h^{-1}
\end{array}\right]: h \in S L(d, \mathbb{R})\right\} \subset D \subset S p(d, \mathbb{R}) \\
S O(d) \hookrightarrow\left\{\left[\begin{array}{cc}
h & 0 \\
0 & { }^{t} h^{-1}
\end{array}\right]: h \in S O(d)\right\} \subset D \subset S p(d, \mathbb{R}) .
\end{gathered}
$$

Exercise 3.3. Show that $D, A$ and $S$ are indeed closed subgroups of $S p(d, \mathbb{R})$. Show that $D$ a normalizes $S$, that is, that $d s d^{-1} \in S$ whenever $d \in D$ and $s \in S$. Describe explicitly the group

$$
S D=\{s d: d \in D, s \in S\}
$$

and exhibit its semidirect product structure. This group is called the standard maximal parabolic subgroup of $S p(d, \mathbb{R})$. Show also that

$$
\bar{S}=\left\{{ }^{t} s: s \in S\right\}
$$

is a subgroup of $S p(d, \mathbb{R})$ and make the appropriate statements for $\bar{S} D$. Prove that $S D$ and $\bar{S} D$ are conjugate.

Proposition 3.4. The symplectic group is generated by $S \cup D \cup\{J\}$ and also by $\bar{S} \cup D \cup\{J\}$.

The meaning of the above statements is that any element in $S p(d, \mathbb{R})$ can be written as a finite product of elements all taken from $S \cup D \cup\{J\}$, or all taken from $\bar{S} \cup D \cup\{J\}$. This fact is of practical relevance, as we shall see, when dealing with the metaplectic representation. 
3.2. Construction of the metaplectic representation. We now proceed to define the metaplectic representation abstractly. Later we indicate how one can go about clearing out all the unsettled points.

Recall that the group $\mathcal{T}$ of automorphisms of the Heisenberg group that leave the center poinwise fixed is generated by the inner automorphisms and by the symplectic maps. More precisely, the maps $i_{h}$ with $h \in \mathbb{H}^{d}$ and $T_{g}$ with $g \in S p(d, \mathbb{R})$ given by

$$
\begin{aligned}
i_{(x, y, z)}(q, p, t) & =(x, y, z)(q, p, t)(x, y, z)^{-1}=(q, p, t+x \cdot p-y \cdot q) \\
T_{g}(q, p, t) & =(g(q, p), t),
\end{aligned}
$$

where $g(q, p)$ simply means the effect of the linear map $g$ on the vector $(q, p) \in \mathbb{R}^{2 d}$.

If $T \in \mathcal{T}$, we can precompose the Schrödinger representation $\pi$ with $T$ to obtain a new representation $\pi \circ T$ of $\mathbb{H}^{d}$ on $L^{2}\left(\mathbb{R}^{d}\right)$. Indeed, if $h, k \in \mathbb{H}^{d}$, then

$$
(\pi \circ T)(h k)=\pi(T(h k))=\pi(T(h) T(k))=\pi(T(h)) \pi(T(k))=(\pi \circ T)(h)(\pi \circ T)(k)
$$

shows that it is indeed a representation and furthermore (2.85) shows that

$$
(\pi \circ T)(0,0, t)=\pi(T(0,0, t))=\pi(0,0, t)=e^{2 \pi i t} I .
$$

By the Stone-von Neumann theorem, $\pi$ and $\pi \circ T$ must be equivalent. Hence there must be a unitary operator $\mu(T)$ on $L^{2}\left(\mathbb{R}^{d}\right)$ that intertwines the two representations:

$$
\pi \circ T(h)=\mu(T) \pi(h) \mu(T)^{-1}, \quad h \in \mathbb{H}^{d} .
$$

Moreover, by Schur's Lemma, namely item ii) of Lemma 1.54, $\mu(T)$ is determined up to a phase factor because the space $\mathcal{I}(\pi, \pi \circ T)$ of all intertwining operators is one dimensional and contains a unitary operator, hence all its multiples by complex numbers of modulus one. Now, if we write (3.105) for the product $T S$ we find that $\mu(T) \mu(S)$ does the job, so there exists $c_{T, S} \in S^{1}$ such that

$$
\mu(T S)=c_{T, S} \mu(T) \mu(S) .
$$

This means that $\mu$ defines a projective representation of $\mathcal{T}$, that is, a homomorphism into the quotient of the group of unitary operators modulo its center $\left\{c I: c \in S^{1}\right\}$.

Now, if $T=i_{h}$, with $h \in \mathbb{H}^{d}$, then since

$$
\pi\left(h g h^{-1}\right)=\pi(h) \pi(g) \pi(h)^{-1},
$$

we can certainly take $\mu\left(i_{h}\right)=\pi(h)$. Thus we may restrict ourselves to the subgroup of $\mathcal{T}$ consisting of the automorphisms $T_{g}$ with $g \in S p(d, \mathbb{R})$, a group manifestly isomorphic to $S p(d, \mathbb{R})$ itself. We shall write for simplicity $\mu(g)$ instead of $\mu\left(T_{g}\right)$. From the above discussion it follows that the unitary operator $\mu(g)$ is determined up to a phase factor by the relation

$$
\pi(g(q, p), 0)=\mu(g) \pi(q, p, 0) \mu(g)^{-1} .
$$

It may be shown (see below for further comments) that the phase factors can be chosen in one and only one way up to a sign, so that $\mu$ becomes a double-valued unitary representation of $S p(d, \mathbb{R})$. In other words, it holds

$$
\mu(g h)= \pm \mu(g) \mu(h), \quad g, h \in S p(d, \mathbb{R}) .
$$


With this choice, $\mu$ is called the metaplectic representation.

We should either think of $\mu$ as a genuine unitary representation of the double cover $\operatorname{Mp}(d)$ of $S p(d, \mathbb{R})$ or as a homomorphism of $S p(d, \mathbb{R})$ into the quotient of the group of unitary operators modulo its center $\{ \pm I\}$. Given a single $g \in S p(d, \mathbb{R})$, we could also think of $\mu(g)$ as a pair of unitary operators that differ from each other by -1 , but we shall not do so. In explicit formulas, this ambiguity usually appears as the possible choice of sign of a square root.

Next we compute $\mu(g)$ for certain particular types of $g$, up to phase factors.

(i) Take $g \in D$, that is $g=\left[\begin{array}{cc}h & 0 \\ 0 & { }^{t} h^{-1}\end{array}\right]$ with $h \in G L(d, \mathbb{R})$. Then

$$
\begin{aligned}
\pi(g(q, p), 0) f(x) & =\pi\left(h q,{ }^{t} h^{-1} p, 0\right) f(x) \\
& =e^{-\pi i(h q) \cdot\left(h^{-1} p\right)} e^{2 \pi i\left({ }^{t} h^{-1} p\right) \cdot x} f(x-h q) \\
& =e^{-\pi i q \cdot p} e^{2 \pi i p \cdot h^{-1} x}(f \circ h)\left(h^{-1} x-q\right)
\end{aligned}
$$

The unitary operator on $L^{2}\left(\mathbb{R}^{d}\right)$ given by $U f(x)=|\operatorname{det} h|^{-1 / 2} f\left(h^{-1} x\right)$ satisfies

$$
\begin{aligned}
U \pi(q, p, 0) U^{-1} f(x) & =|\operatorname{det} h|^{-1 / 2}\left(\pi(q, p, 0) U^{-1} f\right)\left(h^{-1} x\right) \\
& =|\operatorname{det} h|^{-1 / 2}\left(\pi(q, p, 0)|\operatorname{det} h|^{1 / 2} f \circ h\right)\left(h^{-1} x\right) \\
& =\pi(q, p, 0)(f \circ h)\left(h^{-1} x\right) \\
& =e^{-\pi i q \cdot p} e^{2 \pi i p \cdot h^{-1} x}(f \circ h)\left(h^{-1} x-q\right)
\end{aligned}
$$

Hence $U$ satisfies (3.106), so it must coincide with $\mu(g)$ up to a phase factor.

(ii) Take now $g \in S$, that is, $g=\left[\begin{array}{ll}I & 0 \\ \sigma & I\end{array}\right]$ with $\sigma \in \operatorname{Sym}(d, \mathbb{R})$. Then

$$
\begin{aligned}
\pi(g(q, p), 0) f(x) & =\pi(q, \sigma q+p, 0) f(x) \\
& =e^{-\pi i q \cdot(\sigma q+p)} e^{2 \pi i(\sigma q+p) \cdot x} f(x-q) \\
& =e^{-\pi i q \cdot p} e^{2 \pi i p \cdot x} e^{-\pi i q \cdot \sigma q} e^{2 \pi i q \cdot \sigma x} f(x-q)
\end{aligned}
$$

The unitary operator on $L^{2}\left(\mathbb{R}^{d}\right)$ given by $V f(x)=e^{\pi i x \cdot \sigma x} f(x)$ satisfies

$$
\begin{aligned}
V \pi(q, p, 0) V^{-1} f(x) & =e^{\pi i x \cdot \sigma x}\left(\pi(q, p, 0) V^{-1} f\right)(x) \\
& =e^{\pi i x \cdot \sigma x} e^{-\pi i q \cdot p} e^{2 \pi i p \cdot x}\left(V^{-1} f\right)(x-q) \\
& =e^{\pi i x \cdot \sigma x} e^{-\pi i q \cdot p} e^{2 \pi i p \cdot x} e^{-\pi i(x-q) \cdot \sigma(x-q)} f(x-q) \\
& =e^{-\pi i q \cdot p} e^{2 \pi i p \cdot x} e^{-\pi i q \cdot \sigma q} e^{2 \pi i q \cdot \sigma x} f(x-q) .
\end{aligned}
$$

Hence $V$ satisfies (3.106), so it must coincide with $\mu(g)$ up to a phase factor. (iii) Finally, take $g=J$. Then

$$
\pi(J(q, p), 0) f(\xi)=\pi(p,-q, 0) f(\xi)=e^{\pi i q \cdot p} e^{-2 \pi i q \cdot \xi} f(\xi-p)
$$


Denote the Fourier transform on $L^{2}\left(\mathbb{R}^{d}\right)$ by $\mathcal{F}$. For $f \in L^{2}\left(\mathbb{R}^{d}\right)$ we have

$$
\begin{aligned}
\mathcal{F} \pi(q, p, 0) \mathcal{F}^{-1} f(\xi) & =\int e^{-2 \pi i \xi \cdot x} \pi(q, p, 0)\left(\mathcal{F}^{-1} f\right)(x) d x \\
& =\int e^{-2 \pi i \xi \cdot x} e^{-\pi i q \cdot p} e^{2 \pi i p \cdot x}\left(\mathcal{F}^{-1} f\right)(x-q) d x \\
& =e^{-\pi i q \cdot p} \int e^{2 \pi i(p-\xi) \cdot x}\left(\mathcal{F}^{-1} f\right)(x-q) d x \\
& =e^{-\pi i q \cdot p} \int e^{2 \pi i(p-\xi) \cdot(q+y)}\left(\mathcal{F}^{-1} f\right)(y) d y \\
& =e^{-\pi i q \cdot p} e^{2 \pi i(p-\xi) \cdot q} \int e^{2 \pi i(p-\xi) \cdot y}\left(\mathcal{F}^{-1} f\right)(y) d y \\
& =e^{\pi i q \cdot p} e^{-2 \pi i \xi \cdot q} f(\xi-p) .
\end{aligned}
$$

Hence $\mathcal{F}$ satisfies (3.106), so it must coincide with $\mu(g)$ up to a phase factor.

In summary, up to phase factors, we have identified $\mu(g)$ on a generating set of elements (thanks to Proposition 3.4), so in principle we know $\mu$ up to phase factors. Anticipating what can be made rigorous, we actually have

$$
\begin{gathered}
\mu\left(\left[\begin{array}{cc}
h & 0 \\
0 & { }^{t} h^{-1}
\end{array}\right]\right) f(x)=(\operatorname{det} h)^{-1 / 2} f\left(h^{-1} x\right) \\
\mu\left(\left[\begin{array}{ll}
I & 0 \\
\sigma & I
\end{array}\right]\right) f(x)= \pm e^{\pi i x \cdot \sigma x} f(x) \\
\mu(J) f(x)=i^{d / 2} \mathcal{F} f(x) .
\end{gathered}
$$

It must be pointed out that in formulae (3.107) and (3.109) the sign of the square root accounts exactly for the ambiguity in sign.

Exercise 3.5. Show that both the even and odd parts of $L^{2}$, namely

$$
\begin{aligned}
& L_{e}^{2}\left(\mathbb{R}^{d}\right)=\left\{f \in L^{2}\left(\mathbb{R}^{d}\right): f(-x)=f(x)\right\} \\
& L_{o}^{2}\left(\mathbb{R}^{d}\right)=\left\{f \in L^{2}\left(\mathbb{R}^{d}\right): f(-x)=-f(x)\right\},
\end{aligned}
$$

are closed invariant subspaces on each of which the action of $\mu$ is irreducible.

3.2.1. An outline of the full construction. The question remains: how to define $\mu$ in full detail? Our next target is to outline how to do this. The several nontrivial technicalities will not be unravelled completely. The construction can be summarized in the following three basic steps, for each of which we will then give some further information.

(i) Define first the infinitesimal representation $d \mu$ of the Lie algebra $\mathfrak{s p}(d, \mathbb{R})$ by densely defined unbounded and essentially skew-adjoint operators on $L^{2}\left(\mathbb{R}^{d}\right)$. This step is obtained by means of the so called Weyl calculus, realizing first $\mathfrak{s p}(d, \mathbb{R})$ as a Lie algebra of polynomials.

(ii) "Integrate" the representation to a representation of the universal cover of $S p(d, \mathbb{R})$. This step uses the notion of analytic vector for a representation. 
(iii) Show that the representation actually factors to a representation of the double cover of $S p(d, \mathbb{R})$.

3.3. Restriction to triangular subgroups. We finally present the class of examples that has been looked at in the papers ??????

Definition 3.6. A Lie subgroup $G$ of $S p(d, \mathbb{R})$ belongs to the class $\mathcal{E}$ if it is of the form

$$
G=\left\{\left[\begin{array}{cc}
h & 0 \\
\sigma h & { }^{t} h^{-1}
\end{array}\right]: h \in H, \sigma \in \Sigma\right\},
$$

where $H$ is a connected Lie subgroup of $G L(d, \mathbb{R})$ and $\Sigma$ is a subspace of the space $\operatorname{Sym}(d, \mathbb{R})$ of $d \times d$ symmetric matrices. We further require that both $\Sigma$ and $H$ are not trivial.

In order for $G$ to be a group it is necessary and sufficient that $h^{\dagger}[\Sigma]=\Sigma$ for all $h \in H$, where

$$
h^{\dagger}[\sigma]={ }^{t} h^{-1} \sigma h^{-1}
$$

If $G \in \mathcal{E}$, both $\Sigma$ and $H$ are naturally identified as Lie subgroups of $G$. Clearly, $\Sigma H=G, \Sigma \cap H=\{e\}, \Sigma$ is a normal subgroup of $G$ and it is invariant under the action of $H$ given by (3.110), so that $G$ is the semi-direct product $\Sigma \rtimes H$.

Suppose that $G=\Sigma \rtimes H$ is in the class $\mathcal{E}$. A left Haar measure and the modular function of $G$ are

$$
d g=\chi(h)^{-1} d \sigma d h \quad \Delta_{G}(\sigma, h)=\chi(h)^{-1} \Delta_{H}(h),
$$

where $d \sigma$ is a Haar measure of $\Sigma, d h$ is a left Haar measure of $H, \Delta_{H}$ is the modular function of $H$, and $\chi$ is the positive character of $H$ given by

$$
\chi(h)=\left|\operatorname{det} \sigma \mapsto h^{\dagger}[\sigma]\right| .
$$

Exercise 3.7. Prove all the statements that have been made in the previous paragraph: $G$ is a group if and only if $h^{\dagger}[\Sigma]=\Sigma$ for all $h \in H$, and the formulae (3.111) do define Haar measure and modular function.

There is a surprisingly large class of groups in the class $\mathcal{E}$ that are relevant in the study of reproducing systems.

Example 3.8. For $d=1$, that is, for $S L(2, \mathbb{R})$, there is a very remarkable example, namely a copy of the " $a x+b$ " group. It is actually completely obvious that, for $d=1$, in the class $\mathcal{E}$ there is exactly one group, that will be denoted $E_{1}$. The map

$$
\varphi((a, b))=\left[\begin{array}{cc}
1 / \sqrt{a} & 0 \\
b / \sqrt{a} & \sqrt{a}
\end{array}\right]
$$

establishes an isomorphism between " $a x+b$ " and the group $E_{1}$, as one checks immediately. Observe that, for the group $E_{1},(3.107)$ is meaningful for the $1 \times 1$ positive matrix $h=1 / \sqrt{a}$ (no sign ambiguity) and if one takes the plus sign in (3.108), then one gets a perfectly well defined unitary representation on $E_{1}$, and it not necessary to appeal neither to projective representations nor to coverings. As we will see, this 
argument holds for all groups in the class $\mathcal{E}$ so that it is legitimate to speak about the metaplectic representation for groups in $\mathcal{E}$, which will again be denoted by $\mu$.

We show next that the restriction of $\mu$ to $E_{1}$ acting on the space of even $L^{2}$ functions on the line is equivalent to the subrepresentation $\pi$ of the wavelet representation (1.32) of " $a x+b$ " on the Hardy space $\mathcal{H}_{-}(\mathbb{R})$ defined in $(1.41)$. In other words, denoting by $\mu^{e}$ the former and by $\pi^{-}$the latter, we will show that

$$
\mu^{e} \simeq \pi^{-} \text {. }
$$

Similarly, $\mu^{o} \simeq \pi^{+}$for the subrepresentations on odd functions and positively supported transforms, respectively, so that in the end we obtain

$$
\left.\mu\right|_{E_{1}} \simeq \pi
$$

This proves that the wavelet representation is given by restricting the metaplectic representation to a suitable subgroup of $S L(2, \mathbb{R})$.

A crucial remark in order to prove the sought for equivalence is that one should think of $\mu$ as acting on Fourier transforms. For this reason, we write $\widehat{\mathbb{R}}$ for the frequency domain, $L^{2}\left(\widehat{\mathbb{R}}_{+}\right)$for the $L^{2}$-transforms that are supported in $\widehat{\mathbb{R}}_{+}$and $L_{e}^{2}(\widehat{\mathbb{R}})$ for the even $L^{2}$-transforms. The map $\chi_{+}: L_{e}^{2}(\widehat{\mathbb{R}}) \rightarrow L^{2}\left(\widehat{\mathbb{R}}_{+}\right)$defined by

$$
\left(\chi_{+} \hat{g}\right)(\xi)=\sqrt{2} \chi_{[0,+\infty)}(\xi) \hat{g}(\xi)
$$

is an obvious unitary isomorphism. Next we put

$$
\Phi: L^{2}\left(\widehat{\mathbb{R}}_{+}\right) \rightarrow L^{2}\left(\widehat{\mathbb{R}}_{+}\right), \quad \Phi(\hat{g})(\xi)= \begin{cases}(2 \xi)^{-1 / 4} \hat{g}(\sqrt{2 \xi}) & \xi \geqslant 0 \\ 0 & \xi<0 .\end{cases}
$$

Clearly, $\Phi$ is unitary as well. Finally, we denote by $\mathcal{R}$ the reflection $\mathcal{R} f(x)=f(-x)$. Since $\mathcal{R}$ commutes with $\mathcal{F}$, it sends $\mathcal{H}_{+}(\mathbb{R})$ unitarily onto $\mathcal{H}_{-}(\mathbb{R})$, and viceversa. We show next that the unitary map

$$
T: L_{e}^{2}(\widehat{\mathbb{R}}) \rightarrow \mathcal{H}_{-}(\mathbb{R}), \quad T=\mathcal{R} \circ \mathcal{F}^{-1} \circ \Phi \circ \chi_{+}
$$

intertwines $\mu^{e}$ and $\pi^{-}$, that is, for every $\hat{f} \in L_{e}^{2}(\widehat{\mathbb{R}})$ it holds

$$
T\left(\mu_{a, b}^{e} \hat{f}\right)=\pi_{a, b}^{-}(T \hat{f}) .
$$

Here we have written for short $\mu_{a, b}^{e}$ in place of $\mu^{e}(\varphi(a, b))$ and $\pi_{a, b}^{-}$instead of $\pi^{-}(a, b)$. Applying the definition, and reflection on both sides, (3.113) is equivalent to

$$
\mathcal{F}^{-1}\left(\Phi \chi_{+}\left(\mu_{a, b}^{e} \hat{f}\right)\right)=\mathcal{R} \pi_{a, b}^{-} \mathcal{R}\left(\mathcal{F}^{-1} \Phi \chi_{+} \hat{f}\right)
$$

which, after Fourier transformation, is in turn equivalent to

$$
\Phi \chi_{+}\left(\mu_{a, b}^{e} \hat{f}\right)(\xi)=\mathcal{F}\left[\mathcal{R} \pi_{a, b}^{-} \mathcal{R}\left(\mathcal{F}^{-1} \Phi \chi_{+} \hat{f}\right)\right](\xi) \quad \text { q.o. } \xi \in \mathbb{R} .
$$

Next we observe that $\mathcal{R} \pi_{a, b} \mathcal{R}$ sends each $\mathcal{H}_{ \pm}(\mathbb{R})$ into itself and satisfies, for $h \in L^{2}(\mathbb{R})$,

$$
\mathcal{R} \pi_{a, b} \mathcal{R} h(x)=\frac{1}{\sqrt{a}} h\left(\frac{x+b}{a}\right)=\pi_{a,-b} h(x) .
$$


Therefore, using this and (1.37), the right hand side of (3.114) becomes

$$
\begin{aligned}
\mathcal{F}\left[\pi_{a,-b}^{-}\left(\mathcal{F}^{-1} \Phi \chi_{+} \hat{f}\right)\right](\xi) & =\sqrt{a} e^{2 \pi i b \xi}\left(\Phi \chi_{+} \hat{f}\right)(a \xi) \\
& =\sqrt{2} \sqrt{a} e^{2 \pi i b \xi}(2 a \xi)^{-1 / 4} \hat{f}(\sqrt{2 a \xi})
\end{aligned}
$$

for $\xi \geqslant 0$, and vanishes for $\xi<0$. The left hand side of (3.114) is

$$
\sqrt{2}(2 \xi)^{-1 / 4} \mu_{a, b}^{e} \hat{f}(\sqrt{2 \xi})=\sqrt{2}(2 \xi)^{-1 / 4} a^{1 / 4} e^{\pi i b(\sqrt{2 \xi})^{2}} \hat{f}(\sqrt{a} \sqrt{2 \xi})
$$

for $\xi \geqslant 0$, and vanishes for $\xi<0$. This establishes (3.113).

As already remarked in the example that we have just discussed, for the groups in $\mathcal{E}$ it is perfectly legitimate to speak about $\mathrm{t} \mu$ as a bona fide representation. The reason is the following. If $\Sigma \rtimes H \in \mathcal{E}$, then $H$ is required to be connected and hence the determinant of all its elements is necessarily positive, which makes (3.107) unambiguous. Secondly, if one takes the plus sign in (3.108) then it is easy to see that

$$
\mu_{(\sigma, h)} f(x)=(\operatorname{det} h)^{-\frac{1}{2}} e^{\pi i\langle\sigma x, x\rangle} f\left(h^{-1} x\right)
$$

is indeed a a unitary representation.

We illustrate next a remarkable struc tural feature of the groups in $\mathcal{E}$ and present a general geometric result.

3.3.1. The symbol. The restriction $\mu$ of the metaplectic representation to $G \in \mathcal{E}$ is completely characterized by a "symbol" $\Phi$, as we now explain. Given ${ }^{3} \omega \in \mathbb{R}^{d}$, the map $\sigma \mapsto-\frac{1}{2}\langle\sigma \omega, \omega\rangle$ is a linear functional on $\Sigma$ and hence it defines a unique element $\Phi(\omega) \in \Sigma^{*}$, the dual of $\Sigma$, by the requirement that

$$
\Phi(\omega)(\sigma)=-\frac{1}{2}\langle\sigma \omega, \omega\rangle_{\mathbb{R}^{d}}
$$

for all $\sigma \in \Sigma$. The corresponding function $\Phi: \mathbb{R}^{d} \rightarrow \Sigma^{*}$ is called the symbol associated to $\Sigma$ and has the invariance property (3.118) that we now explain. Observe first that the contragredient action of (3.110) is given, for $\sigma^{*} \in \Sigma^{*}, \sigma \in \Sigma$, and $h \in H$, by

$$
h\left[\sigma^{*}\right](\sigma)=\sigma^{*}\left(\left(h^{-1}\right)^{\dagger}[\sigma]\right)=\sigma^{*}\left({ }^{t} h \sigma h\right),
$$

Next, notice that the group $H$ acts naturally on $\mathbb{R}^{d}$ by means of

$$
h . \omega=h \omega .
$$

The invariance property that we are after is that for all $\omega \in \mathbb{R}^{d}$ and $h \in H$

$$
\Phi(h . \omega)=h[\Phi(\omega)] .
$$

This is seen by observing that for all $\sigma \in \Sigma$ we have, by (3.117)

$$
\Phi(h . \omega)(\sigma)=-\frac{1}{2}\left\langle{ }^{t} h \sigma h \omega, \omega\right\rangle_{\mathbb{R}^{d}}=\Phi(\omega)\left(\left(h^{-1}\right)^{\dagger}[\sigma]\right)=h[\Phi(\omega)](\sigma) .
$$

Therefore, for $\sigma \in \Sigma$ and $h \in H$, we may rewrite

$$
\mu_{(\sigma, h)} f(\omega)=(\operatorname{det} h)^{-\frac{1}{2}} e^{-2 \pi i \Phi(\omega)(\sigma)} f\left(h^{-1} \omega\right),
$$

\footnotetext{
${ }^{3}$ The reason for the symbol $\omega$ instead of $x$ rests in the fact that we should think of $\mu$ as acting in the frequency domain.
} 
which exhibits $\mu$ as completely determined by the symbol. Also, this proves that $\mu$ is a representation of the kind considered in [10], with a quadratic symbol.

3.3.2. Geometric characterization. The next result gives some necessary and sufficient conditions for a group $G \in \mathcal{E}$ to be reproducing. In order to state it, we need to introduce some standard terminology. First, the set

$$
H[y]=\left\{h[y] \in \mathbb{R}^{n}: h \in H\right\}
$$

is called the orbit of $H$ in $\mathbb{R}^{n}$, where we have identified $\Sigma^{*}$ with $\mathbb{R}^{n}$ and where evidently $n=\operatorname{dim} \Sigma$. Secondly the closed Lie group of $H$ defined by

$$
H_{y}=\{h \in H: h[y]=y\}
$$

is called the stability subgroup at $y \in \mathbb{R}^{n}$. Thirdly, we recall that a subset $A$ in a topological space $X$ is locally closed if it is the intersection of an open and a close set of $X$. Equivalently, if each point in $A$ has an open neighborhood $U \subset X$ such that $A$ is closed in $U$ (with the subspace topology), or, yet equivalently, if $A$ is open in its closure (with the subspace topology). Finally, let $D \Phi(\omega)$ denote the $n \times d$ Jacobian

$$
D \Phi(\omega)=\frac{\partial\left(\varphi_{1}, \ldots, \varphi_{n}\right)}{\partial\left(\omega_{1}, \ldots, \omega_{d}\right)}
$$

and by $J \Phi(\omega)=\sqrt{\operatorname{det} D \Phi(\omega){ }^{t} D \Phi(\omega)}$ the Jacobian determinant. With this notation, the critical points of $\Phi$ are precisely the solutions of the equation $J \Phi(\omega)=0$.

Theorem 3.9. [10,2] Take $G=\Sigma \rtimes H \in \mathcal{E}$ and assume that the orbit $H[y]$ is locally closed in $\mathbb{R}^{n}$ for every $y \in \mathbb{R}^{n}$. If $G$ is a reproducing group, then

i) $G$ is non-unimodular;

ii) $\operatorname{dim} \Sigma \leqslant d$;

iii) the set of critical points of $\Phi$, which is an $H$-invariant closed subset of $\mathbb{R}^{d}$, has zero Lebesgue measure.

Furthermore, if $\operatorname{dim} \Sigma=d$, then

iv) for almost every $y \in \Phi\left(\mathbb{R}^{d}\right)$ the stability subgroup $H_{y}$ is compact.

Conversely, if i), ii) iii) and iv) (without assuming $\operatorname{dim} \Sigma=d$ ) hold true, then $G$ is reproducing.

If we look at what happens when $d=2$, we find that there is a wealth of reproducing systems arising from groups in $\mathcal{E}$ : there are in fact 16 families of inequivalent reproducing groups. For a full discussion, the reader is referred to [1, 2], where these groups are classified up to the appropriate notion of equivalence and where the admissibility conditions analogous to the Calderón equation (1.43) are derived. Here we content ourselves with two examples that underline why this class is interesting.

Example 3.10. (Shearlets.) The Heisenberg group can be realized inside $S p(2, \mathbb{R})$ in a slightly different way from (2.75), which entails a direct realization of the standard product given in (2.74). By this we mean that the latter can be read off from the matrix product of the matrices (2.75), and conversely. There is, however, another standard realization of the Heisenberg group, known as the polarized version $\mathbb{H}_{\text {pol }}^{1}$, that yields an embedding in the symplectic group as an element of the class $\mathcal{E}$. 
The polarized Heisenberg group may be seen as the set of the $3 \times 3$ matrices

$$
g_{\mathrm{pol}}(q, p, t)=\left[\begin{array}{lll}
1 & p & t \\
0 & 1 & q \\
0 & 0 & 1
\end{array}\right],
$$

which obey the product rule

$$
g_{\mathrm{pol}}(q, p, t) g_{\mathrm{pol}}(q, p, t)=g_{\mathrm{pol}}\left(q+q^{\prime}, p+p^{\prime}, t+t^{\prime}+p q^{\prime}\right) .
$$

Exercise 3.11. Check that (3.121) is true and prove that

$$
\varphi: \mathbb{H}^{1} \rightarrow \mathbb{H}_{\text {pol }}^{1}, \quad \varphi(g(q, p, t))=g_{\text {pol }}\left(q, p, t+\frac{q p}{2}\right)
$$

is a group isomorphism. Define $\mathbb{H}_{\text {pol }}^{d}$, and extend all the above to general $d$.

Next we show how to see $\mathbb{H}_{\text {pol }}^{1}$ as a group in $\mathcal{E}$. For this purpose, put

$$
\Sigma=\left\{\left[\begin{array}{cc}
t & -q / 2 \\
-q / 2 & 0
\end{array}\right]: t, q \in \mathbb{R}\right\}, \quad H=\left\{\left[\begin{array}{ll}
1 & 0 \\
p & 1
\end{array}\right]: p \in \mathbb{R}\right\} .
$$

Then

$$
e(q, p, t)=\left[\begin{array}{cc}
h & 0 \\
\sigma h & { }^{t} h^{-1}
\end{array}\right]=\left[\begin{array}{cccc}
1 & 0 & 0 & 0 \\
p & 1 & 0 & 0 \\
t-q p / 2 & -q / 2 & 1 & -p \\
-q / 2 & 0 & 0 & 1
\end{array}\right]
$$

and it is immediate to check that

$$
e(q, p, t) e(q, p, t)=e\left(q+q^{\prime}, p+p^{\prime}, t+t^{\prime}+p q\right),
$$

as required to see that this is an embedding of the polarized group. Finally, we show that the extended (polarized) Heisenberg group (see Exercise 2.12) can also be seen as an element in the class $\mathcal{E}$. This is achieved by extending $H$ to

$$
H_{e}=\left\{\left[\begin{array}{ll}
1 / \sqrt{a} & 0 \\
p / \sqrt{a} & 1
\end{array}\right]: a>0, p \in \mathbb{R}\right\} .
$$

By doing so, one gets the matrices

$$
e(a, q, p, t)=\left[\begin{array}{cccc}
1 / \sqrt{a} & 0 & 0 & 0 \\
p / \sqrt{a} & 1 & 0 & 0 \\
t / \sqrt{a}-q p / 2 \sqrt{a} & -q / 2 & \sqrt{a} & -p \\
-q / 2 \sqrt{a} & 0 & 0 & 1
\end{array}\right]
$$

that satisfy the product rule

$$
e(a, q, p, t) e\left(a, q^{\prime}, p^{\prime}, t\right)=e\left(a a, q+\sqrt{a} q^{\prime}, p+\sqrt{a} p^{\prime}, t+a t^{\prime}+\sqrt{a} p q^{\prime}\right) .
$$

For brevity, we shall denote by $\mathbb{H}_{e}^{1}=\Sigma \rtimes H_{e}$ the group consisting of all the elements in the form (3.124). Evidently, $\mathbb{H}_{e}^{1} \in \mathcal{E}$. Observe that

$$
e(a, 0,0,0) e(1, q, p, t) e(a, 0,0,0)^{-1}=e(1, \sqrt{a} q, \sqrt{a} p, a t),
$$


so that we are indeed extending the Heisenberg group via its homogeneous dilations. We now compute the various ingredients that are needed. First of all, we write

$$
\sigma_{(t, q)}=\left[\begin{array}{cc}
t & -q / 2 \\
-q / 2 & 0
\end{array}\right]
$$

thereby identifying $\Sigma$ with $\mathbb{R}^{2}$. The elements of $\Sigma^{*}$ are in turn identified with vectors in $\mathbb{R}^{2}$ in the sense that to $y \in \mathbb{R}^{2}$ there corresponds the functional $\sigma_{y}^{*}$ for which

$$
\sigma_{y}^{*}\left(\sigma_{(t, q)}\right)=y \cdot(t, q) .
$$

Secondly,

$$
\begin{aligned}
h_{a, p}^{\dagger}\left[\sigma_{(t, q)}\right] & ={ }^{t} h_{a, p}{ }^{-1} \sigma_{(t, q)} h_{a, p}^{-1} \\
& =\left[\begin{array}{cc}
\sqrt{a} & -p \\
0 & 1
\end{array}\right]\left[\begin{array}{cc}
t & -q / 2 \\
-q / 2 & 0
\end{array}\right]\left[\begin{array}{cc}
\sqrt{a} & -p \\
0 & 1
\end{array}\right] \\
& =\left[\begin{array}{cc}
a t+\sqrt{a} p q & -q \sqrt{a} / 2 \\
-q \sqrt{a} / 2 & 0
\end{array}\right]
\end{aligned}
$$

Thus, $h^{\dagger}\left[\sigma_{(t, q)}\right]$ is the element of $\Sigma$ associated to the vector:

$$
M_{a, p}\left[\begin{array}{l}
t \\
q
\end{array}\right]:=\left[\begin{array}{cc}
a & p \sqrt{a} \\
0 & \sqrt{a}
\end{array}\right]\left[\begin{array}{l}
t \\
q
\end{array}\right] \in \mathbb{R}^{2} .
$$

This means that the action $h_{a, p}^{\dagger}$ is expressed in $\mathbb{R}^{2}$ by the matrix $M_{a, p}$. Therefore, the positive character defined in (3.112) is

$$
\chi\left(h_{a, p}\right)=a^{3 / 2} \text {. }
$$

By (3.111), we then have that

$$
\Delta_{\mathbb{H}_{e}^{1}}(e(a, q, p, t))=a^{-3 / 2} \Delta_{H}\left(h_{a, p}\right)
$$

and since $\Delta_{H}\left(h_{a, p}\right)=a^{-1}$, as it is easily checked computing for example the adjoint representation of $H$ on its Lie algebra, we infer that $G$ is not unimodular. Next, since the contragredient action $y \mapsto h[y]$ corresponds to ${ }^{t} M_{a, p}^{-1}$, we have

$$
\left.h_{a, p}\left[\begin{array}{l}
y_{1} \\
y_{2}
\end{array}\right]\right]=\left[\begin{array}{cc}
1 / a & 0 \\
-p / a & 1 / \sqrt{a}
\end{array}\right]\left[\begin{array}{l}
y_{1} \\
y_{2}
\end{array}\right]=\left[\begin{array}{c}
y_{1} / a \\
-p y_{1} / a+y_{2} / \sqrt{a}
\end{array}\right] .
$$

We next compute the symbol $\Phi$. Since

$$
-\frac{1}{2}\left\langle\sigma_{(t, q)} \omega, \omega\right\rangle=-\frac{1}{2}\left[\begin{array}{ll}
\omega_{1} & \omega_{2}
\end{array}\right]\left[\begin{array}{cc}
t & -q / 2 \\
-q / 2 & 0
\end{array}\right]\left[\begin{array}{l}
\omega_{1} \\
\omega_{2}
\end{array}\right]=\left(-\frac{1}{2} \omega_{1}^{2}, \frac{1}{2} \omega_{1} \omega_{2}\right) \cdot(t, q),
$$

the symbol of $\mathbb{H}_{e}^{1}$ is

$$
\Phi\left(\omega_{1}, \omega_{2}\right)=\left(-\frac{1}{2} \omega_{1}^{2}, \frac{1}{2} \omega_{1} \omega_{2}\right)
$$

The Jacobian is easily computed to be

$$
J \Phi\left(\omega_{1}, \omega_{2}\right)=\frac{1}{2} \sqrt{\left(\omega_{1}^{2}-\omega_{1} \omega_{2}\right)\left(\omega_{1}^{2}+\omega_{1} \omega_{2}\right)}
$$

and its zero set is

$$
\left\{\left(\omega_{1}, \omega_{2}\right) \in \mathbb{R}^{2}: \omega_{1}=0\right\} \cup\left\{\left(\omega_{1}, \omega_{2}\right) \in \mathbb{R}^{2}: \omega_{1}= \pm \omega_{2}\right\},
$$


a set of Lebesgue measure zero. The image of $\Phi$ is the closed left half plane.

It is readily seen that the action $(3.126)$ has five orbits in $\mathbb{R}^{2}$ : the origin, the two half lines $\left\{\left(0, y_{2}\right): y_{2}>0\right\}$ and $\left\{\left(0, y_{2}\right): y_{2}<0\right\}$ and the two half spaces $\left\{\left(y_{1}, y_{2}\right): y_{1}>0\right\}$ and $\left\{\left(y_{1}, y_{2}\right): y_{1}>0\right\}$. Each of these sets is locally closed because it is the intersection of a closed and an open set in the plane.

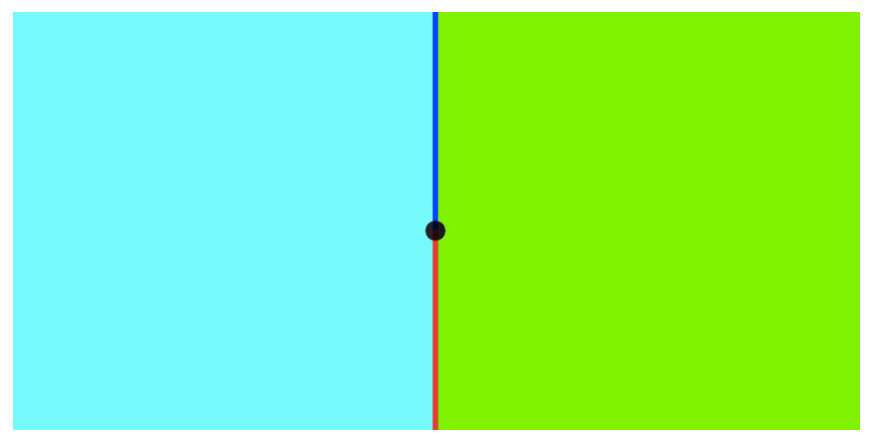

FiguRE 1. The five orbits of $H$

Formula (3.126) also allows us to compute, for every $y \in \Phi\left(\mathbb{R}^{2}\right)$ the stability group $H_{y}$, which is evidently the identity matrix, hence compact, because

$$
\left.h_{a, p}\left[\begin{array}{l}
y_{1} \\
y_{2}
\end{array}\right]\right]=\left[\begin{array}{l}
y_{1} \\
y_{2}
\end{array}\right] \Longleftrightarrow a=1, \quad p=0 \Longleftrightarrow h_{a, p}=I_{2} .
$$

Therefore, all the hypotheses of Theorem 3.9 are satisfied and we may conclude that the restriction ${ }^{4}$ of $\mu$ to to $\mathbb{H}_{e}^{1}$, which by (3.115) or (3.119) is just

$$
\mu(a, q, p, t) \hat{f}\left(\omega_{1}, \omega_{2}\right)=a^{1 / 4} e^{-\pi i(t, q) \cdot\left(-\omega_{1}^{2}, \omega_{1} \omega_{2}\right)} \hat{f}\left(\sqrt{a} \omega_{1}, \omega_{2}-p \omega_{1}\right),
$$

is reproducing. This fact had been established directly in [7] (that is, without the use of Theorem 3.9) and can also be found in [2]. In both papers, Calderón equations for the admissible vectors are also worked out.

A very remarkable fact, one which is particularly relevant for this book, is the following theorem, that is proved in [11]. For the reader's convenience, we give below a direct argument.

Theorem 3.12. [11] The metaplectic representation restricted to the extended Heisenberg group $\mathbb{H}_{e}^{1}$ is equivalent to the shearlet representation of the connected Shearlet group.

First of all, we introduce the connected shearlet group $\mathbb{S}_{+}$that will be discussed in detail in the other chapters of this book, to which we refer for historical and bibliographical information. It is the set $\mathbb{R}_{+} \times \mathbb{R} \times \mathbb{R}^{2}$ endowed with the group operation

$$
(a, s, t)\left(a^{\prime}, s^{\prime}, t^{\prime}\right)=\left(a a^{\prime}, s+a^{1 / 2} s^{\prime}, t+S_{s} A_{a} t^{\prime}\right),
$$

\footnotetext{
${ }^{4}$ Once more, we think of the metaplectic representation as acting on the frequency side.
} 
where

$$
S_{s}=\left[\begin{array}{ll}
1 & s \\
0 & 1
\end{array}\right], \quad A_{a}=\left[\begin{array}{cc}
a & 0 \\
0 & \sqrt{a}
\end{array}\right], \quad S_{s} A_{a}=\left[\begin{array}{cc}
a & s \sqrt{a} \\
0 & \sqrt{a}
\end{array}\right]
$$

We can thus write the group law more explicitly as

$$
(a, s, t)\left(a^{\prime}, s^{\prime}, t^{\prime}\right)=\left(a a^{\prime}, s+a^{1 / 2} s^{\prime}, t_{1}+a t_{1}^{\prime}+\sqrt{a} t_{2}^{\prime}, t_{2}+\sqrt{a} t_{2}^{\prime}\right) .
$$

In order to understand the statement of Theorem 3.12, we observe that the map

$$
\Psi: \mathbb{S}_{+} \rightarrow \mathbb{H}_{e}^{1}, \quad\left(a, s, t_{1}, t_{2}\right) \mapsto e\left(a, t_{2}, s, t_{1}\right)
$$

which amounts to renaming the variables according to

$$
p \longleftrightarrow s, \quad t_{1} \longleftrightarrow t, \quad t_{2} \longleftrightarrow q,
$$

establishes a Lie group isomorphism. Secondly, the shearlet representation on the frequency domain, that is, on $L^{2}\left(\widehat{\mathbb{R}}^{2}\right)$ is defined by:

$$
\hat{\psi}_{(a, s, t)}\left(\omega_{1}, \omega_{2}\right)=a^{3 / 4} e^{-2 \pi i t \cdot \omega} \hat{\psi}\left(a \omega_{1}, \sqrt{a}\left(s \omega_{1}+\omega_{2}\right)\right) .
$$

Renaming the variables after (3.129), this becomes

$$
\hat{\psi}_{(a, q, p, t)}\left(\omega_{1}, \omega_{2}\right)=a^{3 / 4} e^{-2 \pi i(t, q) \cdot\left(\omega_{1}, \omega_{2}\right)} \hat{\psi}\left(a \omega_{1}, \sqrt{a}\left(p \omega_{1}+\omega_{2}\right)\right) .
$$

We now proceed to show the claimed equivalence..

Proof. (of Theorem 3.12.) The first remark concerns irreducibility. For simplicity, put

$$
\Omega_{+}=\left\{\left(\omega_{1}, \omega_{2}\right) \in \widehat{\mathbb{R}}^{2}: \omega_{1}>0\right\}, \quad \Omega_{-}=\left\{\left(\omega_{1}, \omega_{2}\right) \in \widehat{\mathbb{R}}^{2}: \omega_{1}<0\right\} .
$$

It is clear that the spaces $L^{2}\left(\Omega_{+}\right)$and $L^{2}\left(\Omega_{-}\right)$are closed invariant spaces both for the shearlet representation and for the metaplectic representation (3.127). Furthermore, the subrepresentations obtained by restriction to $L^{2}\left(\Omega_{ \pm}\right)$are mutually equivalent, because the reflection $\mathcal{R} \hat{f}\left(\omega_{1}, \omega_{2}\right)=\hat{f}\left(-\omega_{1}, \omega_{2}\right)$ sends one space unitarily into the other, and intertwines the two pairs of subrepresentations. Further, it may be shown that each such subrepresentation is in fact irreducible. Finally, there is an intertwining operator $\mathcal{L}$ that realizes a unitary equivalence between the restriction to $L^{2}\left(\Omega_{-}\right)$of the shearlet representation with the restriction to $L^{2}\left(\Omega_{+}\right)$of $\mu$. The interesting fact is that $\mathcal{L}$ is essentially constructed by means of the symbol $\Phi: \Omega_{+} \rightarrow \Omega_{-}$, which is a diffeomorphism with Jacobian determinant $\omega_{1}^{2} / 2$. We define

$$
\mathcal{L}: L^{2}\left(\Omega_{-}\right) \rightarrow L^{2}\left(\Omega_{+}\right), \quad \mathcal{L} \hat{\varphi}(\omega)=\frac{\omega_{1}}{\sqrt{2}} \hat{\varphi}(\Phi(\omega))
$$

For any $\hat{\varphi} \in L^{2}\left(\Omega_{-}\right)$, we have

$$
\int_{\Omega_{+}}|\mathcal{L} \hat{\varphi}(\omega)|^{2} d \omega=\int_{\Omega_{+}}\left|\frac{\omega_{1}}{\sqrt{2}} \hat{\varphi}(\Phi(\omega))\right|^{2} d \omega=\int_{\Omega_{+}}|\hat{\varphi}(\Phi(\omega))|^{2} \frac{\omega_{1}^{2}}{2} d \omega=\int_{\Omega_{-}}|\hat{\varphi}(\omega)|^{2} d \omega,
$$


so that $\mathcal{L}$ is unitary. Using (3.131) we have

$$
\begin{aligned}
\mathcal{L}\left(\hat{\psi}_{(a, q, p, t)}\right)\left(\omega_{1}, \omega_{2}\right) & =\frac{\omega_{1}}{\sqrt{2}} \hat{\psi}_{(a, q, p, t)}(\Phi(\omega)) \\
& =\frac{\omega_{1}}{\sqrt{2}} \hat{\psi}_{(a, q, p, t)}\left(-\frac{\omega_{1}^{2}}{2}, \frac{\omega_{1} \omega_{2}}{2}\right) \\
& =\frac{\omega_{1}}{\sqrt{2}} a^{3 / 4} e^{-\pi i(t, q)\left(-\omega_{1}^{2}, \omega_{1} \omega_{2}\right)} \hat{\psi}\left(-a \frac{\omega_{1}^{2}}{2}, \sqrt{a}\left(-p \frac{\omega_{1}^{2}}{2}+\frac{\omega_{1} \omega_{2}}{2}\right)\right),
\end{aligned}
$$

and using (3.127) we have

$$
\begin{aligned}
\mu(a, q, p, t)(\mathcal{L} \hat{\psi})\left(\omega_{1}, \omega_{2}\right) & =a^{1 / 4} e^{-\pi i(t, q) \cdot\left(-\omega_{1}^{2}, \omega_{1} \omega_{2}\right)}(\mathcal{L} \hat{\psi})\left(\sqrt{a} \omega_{1}, \omega_{2}-p \omega_{1}\right) \\
& =a^{1 / 4} e^{-\pi i(t, q) \cdot\left(-\omega_{1}^{2}, \omega_{1} \omega_{2}\right)} \frac{\sqrt{a} \omega_{1}}{\sqrt{2}} \hat{\psi}\left(-\frac{a \omega_{1}^{2}}{2}, \frac{1}{2}\left(\sqrt{a}\left(\omega_{1} \omega_{2}-p \omega_{1}^{2}\right)\right) .\right.
\end{aligned}
$$

This establishes the intertwining property

$$
\mathcal{L}\left(\hat{\psi}_{(a, q, p, t)}\right)=\mu(a, q, p, t)(\mathcal{L} \hat{\psi})
$$

and the proof of Theorem 3.12.

Example 3.13. (Schrödingerlets.) This is perhaps one of the few examples of a 3dimensional reproducing systems in $L^{2}\left(\widehat{\mathbb{R}}^{2}\right)$. The group $G$ consists of the matrices

$$
\left[\begin{array}{cc}
a^{-1 / 2} R & 0 \\
t a^{-1 / 2} R & a^{1 / 2} R
\end{array}\right], \quad t \in \mathbb{R}, a>0, R \in S O(2) .
$$

The rotations in $S O(2)$ are parametrized in the standard way, namely

$$
R_{\varphi}=\left[\begin{array}{cc}
\cos \varphi & -\sin \varphi \\
\sin \varphi & \cos \varphi
\end{array}\right], \quad \varphi \in[0,2 \pi) .
$$

Therefore $G$ in the class $\mathcal{E}$, with $\Sigma=\left\{t I_{2}: t \in \mathbb{R}\right\}$ and $H=\mathbb{R}_{+} \cdot S O(2)$, an Abelian group. En passant, here $d=2$ and $n=1$. The metaplectic representation $\mu$ restricted to $G$, thought in the frequency domain, is given by formula (3.115), namely

$$
\mu(t, a, \varphi) \hat{f}(\omega)=a^{1 / 2} e^{\pi i t\|\omega\|^{2}} \hat{f}\left(a^{1 / 2} R_{-\varphi} \omega\right), \quad \hat{f} \in L^{2}\left(\widehat{\mathbb{R}}^{2}\right) .
$$

The space-domain version of this representation explains the reason of the name that is used for the admissible vectors relative to this group. Denote by $\tilde{\mu}$ the unitary representation obtained by conjugating $\mu$ with the Fourier transform, that is

$$
\tilde{\mu}(g) f=\mathcal{F}^{-1} \circ \mu(g) \circ \mathcal{F} .
$$

We interpret $t \in \mathbb{R}$ as a time parameter and look at the evolution flow of a function in the space domain $f \in L^{1}\left(\mathbb{R}^{2}\right) \cap L^{2}\left(\mathbb{R}^{2}\right)$, given by

$$
(t, x) \mapsto \tilde{\mu}_{t} f(x)=\tilde{\mu}(t, 1,0) f(x)=\int_{\tilde{\mathbb{R}}^{2}} \hat{f}(\omega) e^{\pi i t\|\omega\|^{2}} e^{2 \pi i x \cdot \omega} d \omega .
$$

It is straightforward to verify that the flow $\tilde{\mu}_{t} f(x)$ satisfies the Schrödinger equation

$$
\left(-4 \pi i \frac{\partial}{\partial t}+\Delta\right) \tilde{\mu}_{t} f(x)=0
$$


where $\Delta$ is the spacial Laplacian

$$
\Delta=\frac{\partial^{2}}{\partial x_{1}^{2}}+\frac{\partial^{2}}{\partial x_{2}^{2}}
$$

For this reason the admissible vectors of $G$ are called Schrödingerlets. In summary, the system of unitary operators attached to $G$ is generated by rotations, by dilations and by the evolution flow of the Schrödinger operator.

We show next that $\mu$ restricted to $G$ is indeed reproducing. First of all, writing $h_{a, \varphi}=a^{-1 / 2} R_{\varphi}$, we have

$$
h_{a, \varphi}^{\dagger}\left[\sigma_{t}\right]={ }^{t} h_{a, \varphi}^{-1} \sigma_{t} h_{a, \varphi}^{-1}=t a I_{2}=\sigma_{a t} .
$$

Thus, the action $h_{a, \varphi}^{\dagger}$ is expressed in $\mathbb{R}$ as multiplication by $a$, so that the contragredient action $y \mapsto h_{a, \varphi}[y]$ amounts to multiplication by $a^{-1}$. It follows that the positive character defined in (3.112) is

$$
\chi\left(h_{a, \varphi}\right)=a .
$$

By (3.111), taking into account that $H$, being Abelian, is unimodular we have that

$$
\left.\Delta_{G}(a, \varphi, t)\right)=a^{-1},
$$

so that $G$ itself is not unimodular. As for the symbol $\Phi: \mathbb{R}^{2} \rightarrow \mathbb{R}$, since

$$
-\frac{1}{2}\left\langle\sigma_{t} \omega, \omega\right\rangle=-\frac{1}{2}\|\omega\|^{2} t
$$

it is the mapping given by

$$
\Phi(\omega)=-\frac{1}{2}\|\omega\|^{2}
$$

Its image is the closed left half line $(-\infty, 0]$ and its Jacobian is

$$
J \Phi\left(\omega_{1}, \omega_{2}\right)=\|\omega\|,
$$

whose zero set is just the origin of $\mathbb{R}^{2}$, obviously of Lebesgue measure zero. The action $y \mapsto h[y]$ has three orbits in $\mathbb{R}$ : the origin and the two half lines $(-\infty, 0)$ and $(0,+\infty)$, each of which is locally closed. For every $y<0$, hence almost everywhere in $\Phi\left(\mathbb{R}^{2}\right)=(-\infty, 0]$, the stability group $H_{y}$ is the identity matrix, hence compact. Therefore, all the hypotheses of Theorem 3.9 are satisfied and $G$ is reproducing. The reader is referred to [2] for the Calderón equations for the admissible vectors.

\section{BIBLIOGRAFY}

[1] G. Alberti, L. Balletti, F. De Mari, F. and E. De Vito. Reproducing subgroups of $S p(2, \mathbb{R})$. Part I: Algebraic classification J. Fourier Anal. Appl. 19 (2013), no. 4, 651-682.

[2] G. Alberti, F. De Mari, F., E. De Vito and L. Mantovani. Reproducing subgroups of $\operatorname{Sp}(2, \mathbb{R})$. Part II: admissible vectors. Monatsh. Math., (2014) 173, n.3, 261-307.

[3] R. Berndt, R. Schmidt, Elements of the representation theory of the Jacobi group. Modern Birkhuser Classics. Basel, 1998.

[4] Nicolas Bourbaki, Integration. I. Chapters 1-6, Elements of Mathematics (Berlin), Springer-Verlag, Berlin, 2004, Translated from the 1959, 1965 and 1967 French originals by Sterling K. Berberian.

[5] E. Cordero, F. De Mari, K. Nowak, and A. Tabacco. Analytic features of reproducing groups for the metaplectic representation. Journal of Fourier Analysis and Applications, 12(2):157-180, 2006. 
[6] E. Cordero, F. De Mari, K. Nowak, and A. Tabacco. Reproducing groups for the metaplectic representation. In P. Boggiatto, L. Rodino, J. Toft, and M. W. Wong, editors, Pseudo-Differential Operators and Related Topics, volume 12 of Operator Theory: Advances and Applications, pages 227-244. Birkhäuser Basel, 2006.

[7] E. Cordero, F. De Mari, K. Nowak, and A. Tabacco. Dimensional upper bounds for admissible subgroups for the metaplectic representation. Mathematische Nachrichten, 283(7):982-993, 2010.

[8] Cordero, E. Tabacco, A., Triangular subgroups of $S p(d, \mathbb{R})$ and reproducing formulae, $J$. Funct. Anal., 264 (2013), no. 9, 2034-2058.

[9] I. Daubechies, Ten lectures on wavelets. SIAM, Philadelphia, 1992.

[10] F. De Mari and E. De Vito. Admissible vectors for mock metaplectic representations. Applied Computational Harmonic Analysis, 34(2):163-200, 2013.

[11] F. De Mari, E. De Vito, S. Dahlke, S. Häuser, G. Steidl and G. Teschke. Different faces of the shearlet group arXiv:1404.4545, 2014.

[12] F. De Mari and K. Nowak. Canonical subgroups of $\mathbb{H}_{1} \rtimes S L(2, \mathbb{R})$, Bollettino U.M.I., (8) 5-B (2002) 405-430

[13] F. De Mari and K. Nowak. Analysis of the affine transformations of the time-frequency plane, Bull. Austral. Math. Soc., Vol. 63 (2001), 195-218.

[14] G. B. Folland, A Course in Abstract Harmonic Analysis, CRC Press, Boca Raton, 1995.

[15] G. B. Folland, Harmonic Analysis in Phase Space, Princeton University Press, Princeton, 1989.

[16] K. Gröchenig, Foundations of Time-Frequency Analysis. Birkhäuser, Boston, 2001.

[17] A.W. Knapp, Lie Groups Beyond an Introduction, Birkhäuser, Boston, 1996.

[18] A.W. Knapp, Representation Theory of Semisimple Lie Groups: An Overview Based on Examples, Princeton University Press, Princeton, 1988.

[19] G. Lion and M. Vergne, The Weil representation, Maslov index and theta series, Birkäuser, Boston, 1980.

[20] W. Rudin, Functional Analysis, second edition, McGraw Hill, New York,1991.

[21] S. Mallat, A wavelet tour of signal processing. Academic Press, San Diego, 1998.

[22] V.S. Varadarajan, Lie Groups, Lie Algebras and Their Representations, second edition, Springer-Verlag, New York, 1984.

[23] F. Warner, Foundations of Differentiable Manifolds and Lie Groups, second edition, Springer-Verlag, New York, 1983.

Filippo De Mari, Università di Genova

E-mail address: demari@dima.unige.it

Ernesto De Vito, Università di Genova

E-mail address: devito@dima.unige.it 